\title{
UWB ULTRA- LOW- POWER SHORT DISTANCE FIR SPECTRALLY SHAPED NRZ TRANSMISSION \\ CHIP-TO-CHIP WIRELESS COMMUNICATION CIRCUIT EXAMPLE
}

by

\author{
Sameera Siddiqui B.E. (Electronics) \\ N.E.D University Of EngG. \& Tech., Karachi, Pakistan
}

A thesis submitted to the Faculty of Graduate Studies \& Research in partial fulfillment of the requirements for the degree of

\section{Master of Applied Science}

\author{
Ottawa-Carleton Institute for Electrical Engineering \\ Department of Electronics \\ Carleton University \\ Ottawa, Ontario \\ June, 2008
}

Copyright (C) 2008, Sameera Siddiqui 


$\begin{array}{ll}\begin{array}{l}\text { Library and } \\ \text { Archives Canada }\end{array} & \begin{array}{l}\text { Bibliothèque et } \\ \text { Archives Canada }\end{array} \\ \begin{array}{l}\text { Published Heritage } \\ \text { Branch }\end{array} & \begin{array}{l}\text { Direction du } \\ \text { Patrimoine de l'édition }\end{array} \\ \begin{array}{l}\text { 395 Wellington Street } \\ \text { Ottawa ON K1A ON4 } \\ \text { Canada }\end{array} & \begin{array}{l}\text { 395, rue Wellington } \\ \text { Ottawa ON K1A 0N4 } \\ \text { Canada }\end{array}\end{array}$

Your file Votre référence ISBN: 978-0-494-44057-5

Our file Notre référence

ISBN: 978-0-494-44057-5

NOTICE:

The author has granted a nonexclusive license allowing Library and Archives Canada to reproduce, publish, archive, preserve, conserve, communicate to the public by telecommunication or on the Internet, loan, distribute and sell theses worldwide, for commercial or noncommercial purposes, in microform, paper, electronic and/or any other formats.

The author retains copyright ownership and moral rights in this thesis. Neither the thesis nor substantial extracts from it may be printed or otherwise reproduced without the author's permission.
AVIS:

L'auteur a accordé une licence non exclusive permettant à la Bibliothèque et Archives Canada de reproduire, publier, archiver, sauvegarder, conserver, transmettre au public par télécommunication ou par l'Internet, prêter, distribuer et vendre des thèses partout dans le monde, à des fins commerciales ou autres, sur support microforme, papier, électronique et/ou autres formats.

L'auteur conserve la propriété du droit d'auteur et des droits moraux qui protège cette thèse. $\mathrm{Ni}$ la thèse ni des extraits substantiels de celle-ci ne doivent être imprimés ou autrement reproduits sans son autorisation.
In compliance with the Canadian

Privacy Act some supporting forms may have been removed from this thesis.

While these forms may be included in the document page count, their removal does not represent any loss of content from the thesis.
Conformément à la loi canadienne sur la protection de la vie privée, quelques formulaires secondaires ont été enlevés de cette thèse.

Bien que ces formulaires aient inclus dans la pagination, il n'y aura aucun contenu manquant.

\section{Canada}




\begin{abstract}
Existing wireless techniques are reviewed with emphasis on ultra wideband and ultra low power solutions. Based on our research group expertise ultra wideband NRZ signaling has been selected as the exploration target. A novel transmitter structure serving as a bridge between high-speed serial data and ultra wideband (UWB) communication is proposed. The circuit is aimed for simple implementation, low power consumption and high data rate. A finite impulse response (FIR) filter is designed and simulated in MATLAB to filter the serial data at the rate of $10 \mathrm{~Gb} / \mathrm{s}$ according to the spectral mask approved by Federal Communication Commission (FCC) with $1.55 \mathrm{GHz}$ to $5 \mathrm{GHz}$ being the targeted bandwidth. Then the entire circuit is designed and simulated in CMOS 90nm technology using current model logic (CML) based structures. Stacked inductors with efficient coupling are used for wireless chip-to-chip transmission. The total layout area of the transmitter is estimated to be of $0.1 \mathrm{~mm} \times 0.13 \mathrm{~mm}$ and it consumes $53.6 \mathrm{~mW}$ with a $1.2 \mathrm{~V}$ supply. The coupling coefficient of inductors is 0.95 and the stacked inductors occupies a layout area of $0.218 \mathrm{~mm}^{2}$. This work accomplishes wireless high speed serial data transfer at $10 \mathrm{Gbps}$ with only $5.36 \mathrm{pJ} / \mathrm{bit}$ energy consumption.
\end{abstract}




\section{Important Information}

The information used in this thesis comes in part from the research program of Dr. Tad Kwasniewski and his research associates in the VLSI in Communications group. The research result appearing in this thesis represents an integral part of the ongoing research program. All research results in this thesis including tables, graphs and figures but excluding narrative portions of the thesis are effectively incorporated into the research program and can be used by Dr. Kwasniewski and his associates for education and research purposes, including publications in open literature with the appropriate credits. Matters of

intellectual property may be pursued cooperatively with Carleton University and Dr. Tad Kwasniewski where and as appropriate. 


\section{Acknowledgments}

I would like to express my deepest gratitude towards my thesis supervisor Dr. Tad Kwasniewski for his guidance and support throughout the research work. His continuous advice, encouragement and help were instrumental in making this research possible and driving it to completion.

I would also like to thank the members of faculty, staff and students of Department of Electronics at Carleton University. I really appreciate the helpful discussions \& suggestions from the members of our research group which made my academic experience much more rewarding. Particularly, I would like to thank Syed Irfan Ahmed, Muhammad Usama and John Cheng for their cooperation and beneficial advice. I wish them all the best for their future endeavors.

Financial support from Ontario Ministry Of Training, Colleges and University and Altera Corporation is gratefully acknowledged.

Finally, I thank my family for their support and understanding. Their continuous encouragement kept me motivated and energized during difficult times. Special gratitude is directed towards my parents who always hearten me, and my wonderful husband Farrukh, without whose support and encouragement this work would have stayed a dream. 


\section{Table Of Contents}

\section{Chapter 1: Introduction}

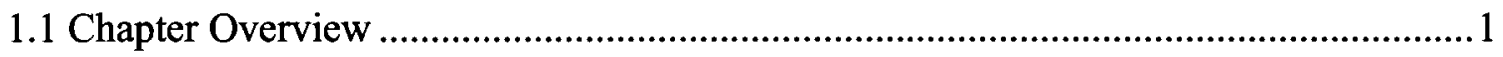

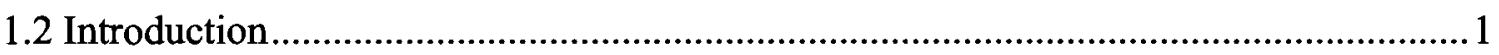

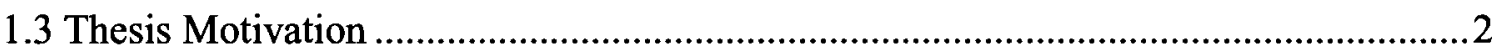

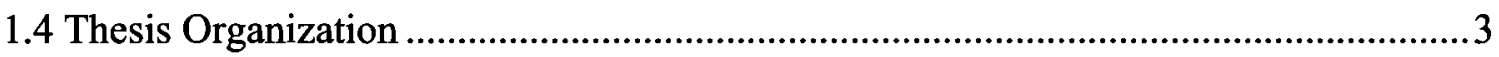

\section{Chapter 2: Background}

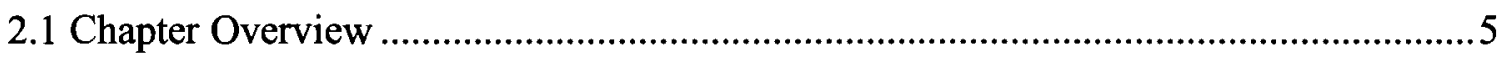

2.2 Introduction To Wireless Sensor Networks ...........................................................

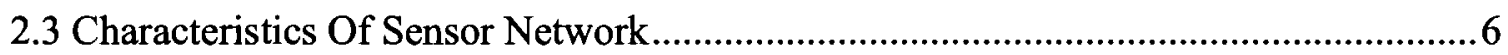

2.3.1 Self-Organization ........................................................................................

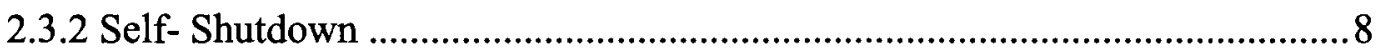

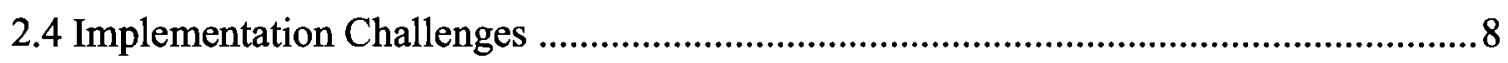

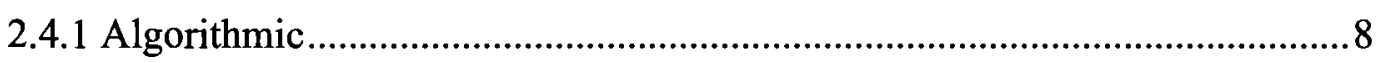

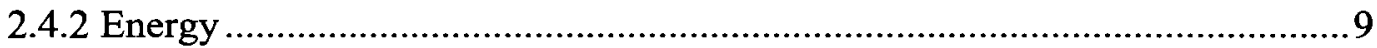

2.5 Introduction To Ultra Wideband Communication ..................................................10

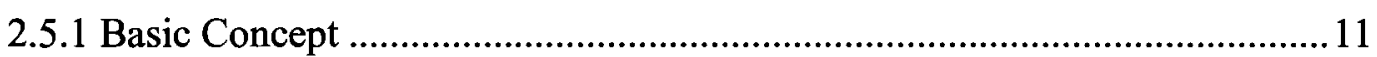


2.6 Advantages Of Ultra Wideband Communication Systems

2.6.1 Frequency Spectrum Sharing ....................................................................14

2.6.2 Robustness Against Eavesdropping ..........................................................14

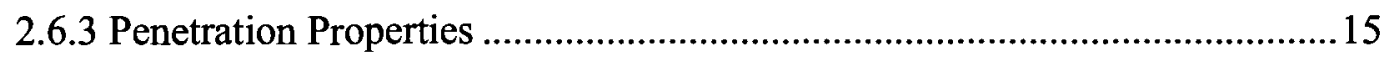

2.6.4 Robustness Against Multipath Fading ………….........................................15

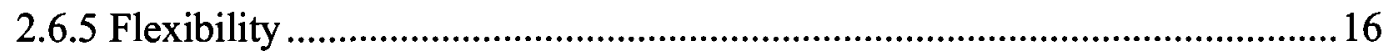

2.6.6 Robustness Against Channels...................................................................17

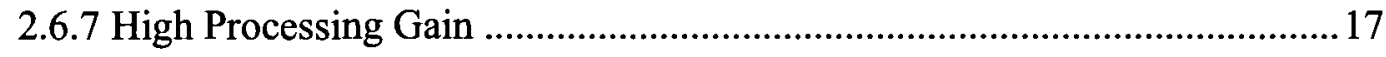

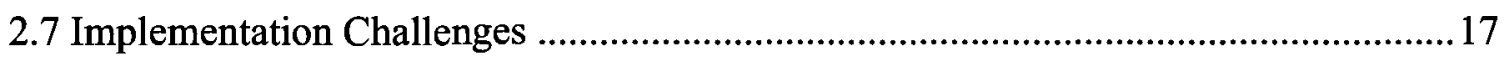

2.7.1 Pulse Shape Distortion ............................................................................ 18

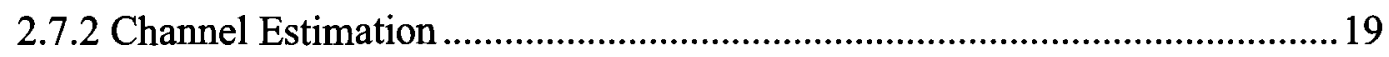

2.7.3 Accurate Synchronization .......................................................................20

2.7.4 High Sampling Rate For Digital Implementation ........................................20

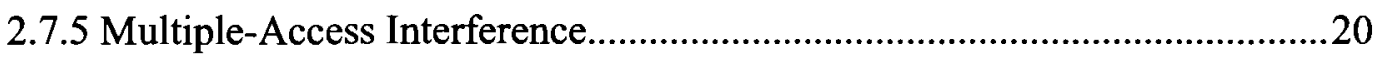

2.7.6 High Performance Transceivers...................................................................21

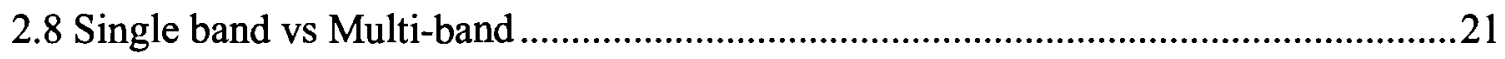

2.8.1 Direct -Sequence UWB …………...........................................................22

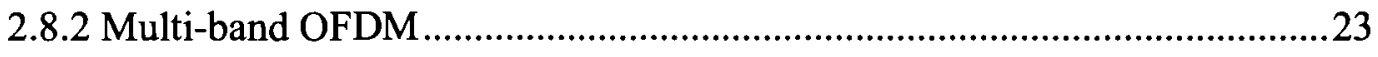

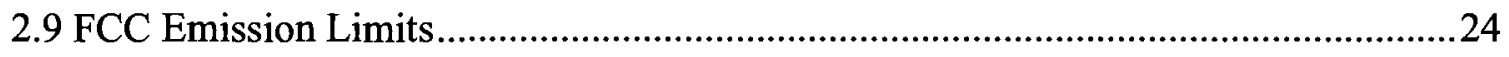


2.9.1 Communication Devices ............................................................................24

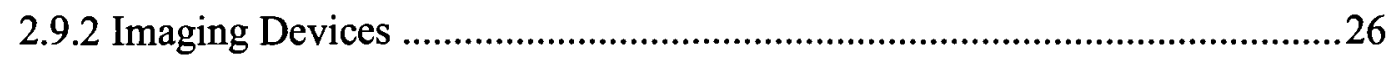

2.9.3 Vehicular Radar System .........................................................................2

2.10 Applications Of Ultra Wideband ....................................................................30

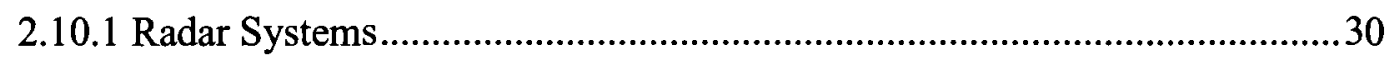

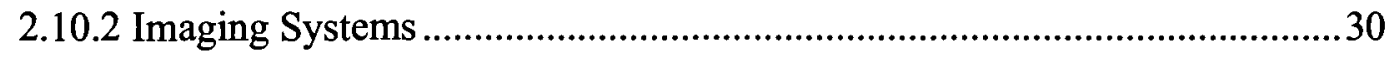

2.10.3 Communication Systems ........................................................................

2.10.4 Wireless Sensor Network Systems ……………….................................

\section{Chapter 3: Design Considerations Of A Wireless UWB Transceiver}

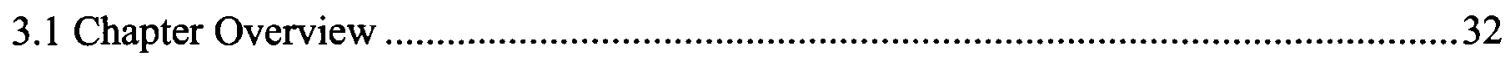

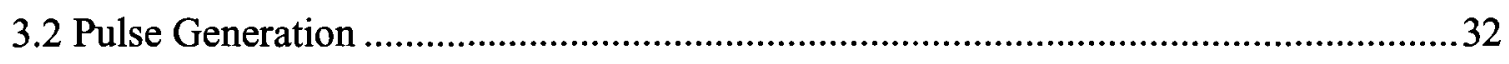

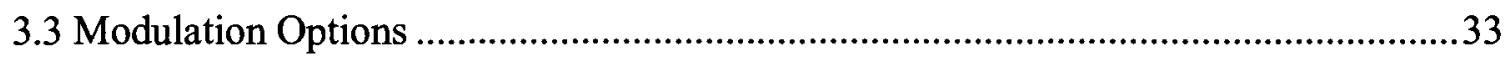

3.3.1 Pulse Amplitude Modulation ..........................................................................

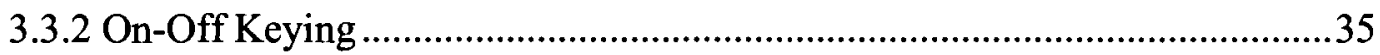

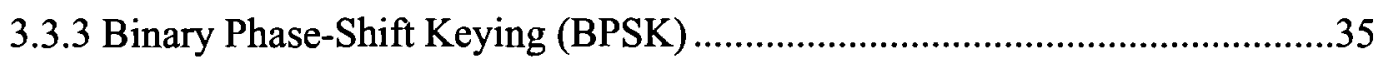

3.3.4 Pulse Position Modulation (PPM) ……………………...............................36

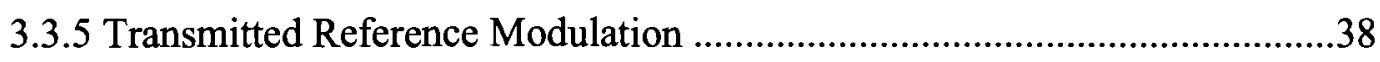

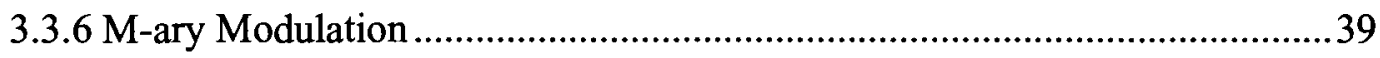

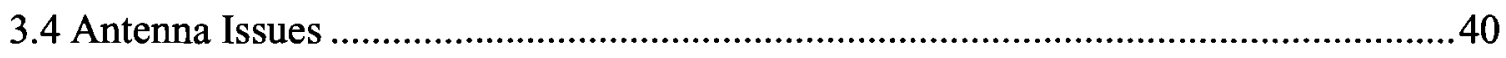

3.5 Impulse Radio Transmitter Design Issues .............................................................. 41 


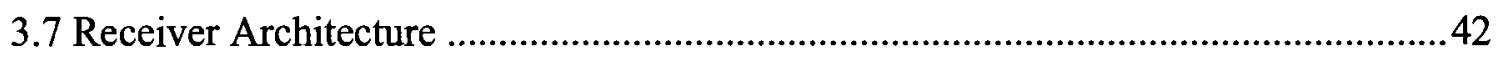

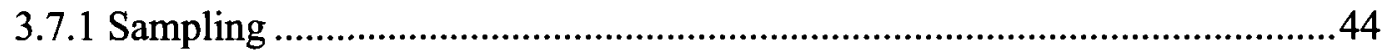

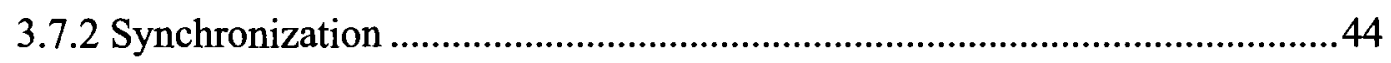

3.7.3 Multipath Coefficient Estimation ............................................................4

3.7.4 Pulse Shape Estimation...............................................................................45

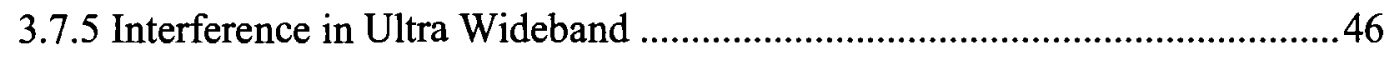

3.7.6 Modulation Options and Application Specific Issues...................................46

3.8 Channel Modeling.............................................................................................

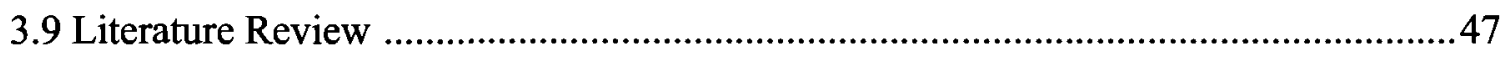

3.10 Proposed Approach For Serial Data Transmission in UWB Spectrum ......................55

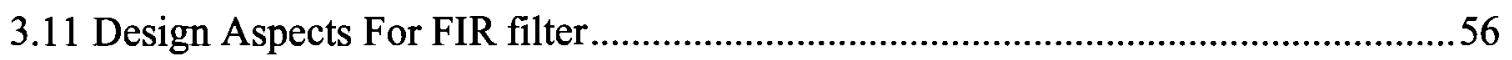

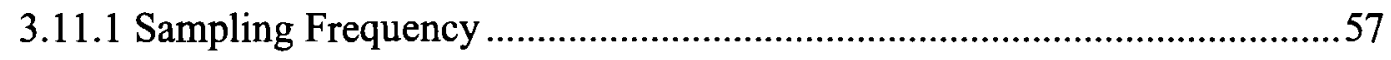

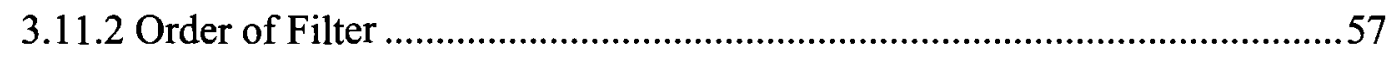

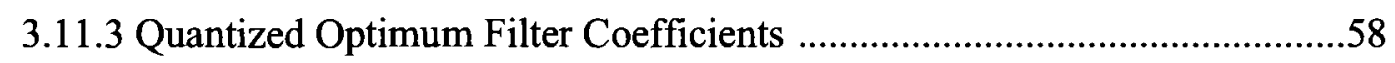

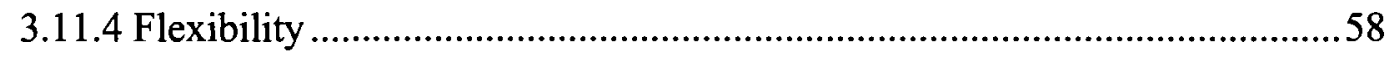

3.12 Design Aspects Of Stacked Inductors .............................................................58

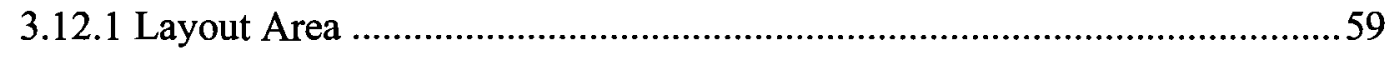

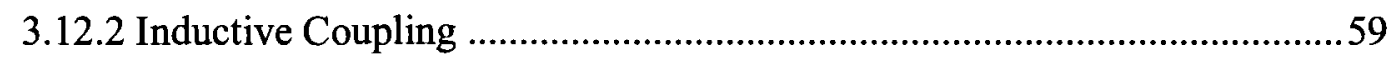

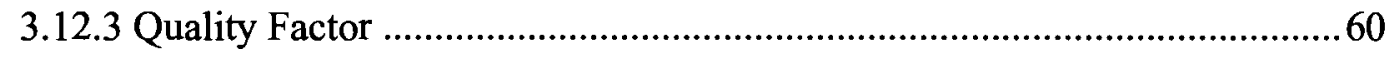




\section{Chapter 4: Design \& Evaluation of UWB Channel}

4.1 Chapter Overview

4.2 Implementation Challenges .61

4.2.1 Meeting Filter Design Specifications and Chip-to-Chip Communication Feasibility in CDE .62

4.3 Channel Modeling in MATLAB .63

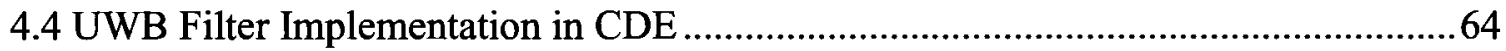

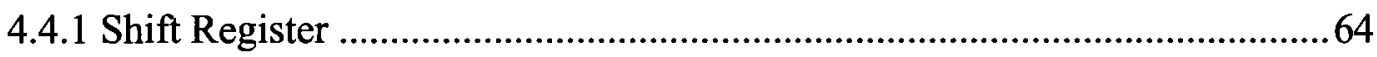

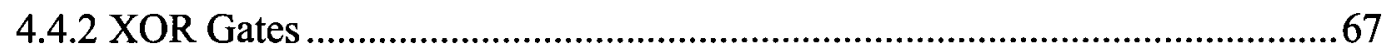

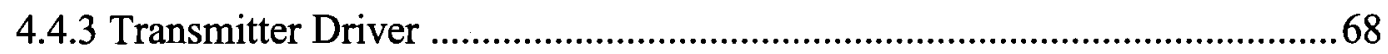

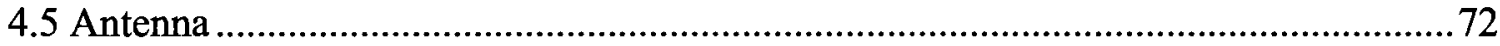

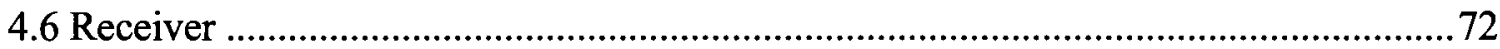

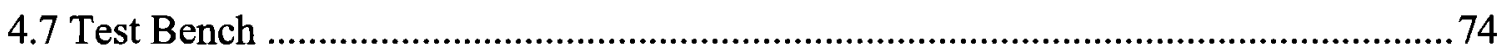

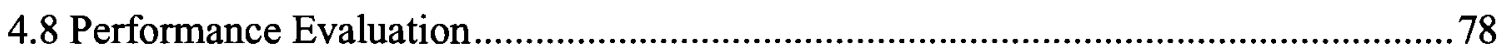

\section{Chapter 5: Simulation Results \& Analysis}

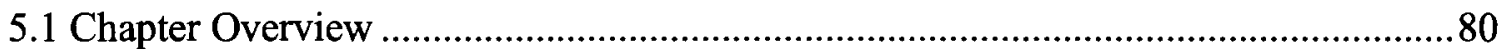

5.2 Optimal Tap Coefficients and Modeled UWB Channel .............................................80

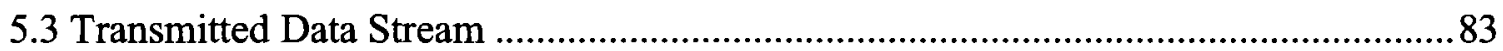

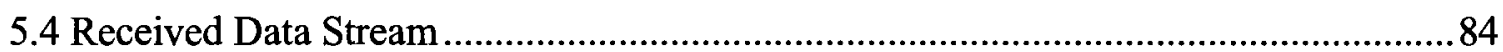


Chapter 6: Conclusions \& Recommendations

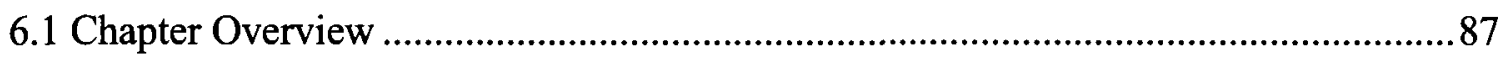

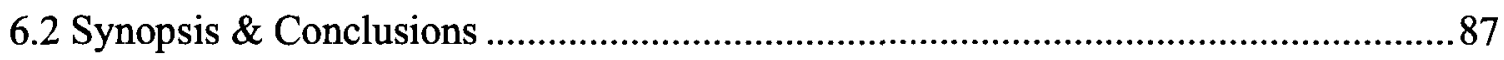

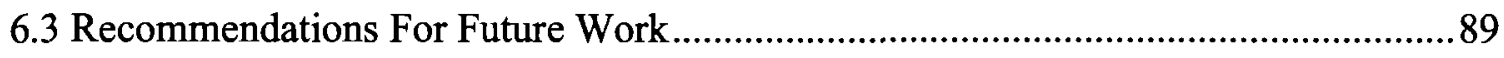

APPENDIX A: FCC Emission Limits Tables .......................................................................90

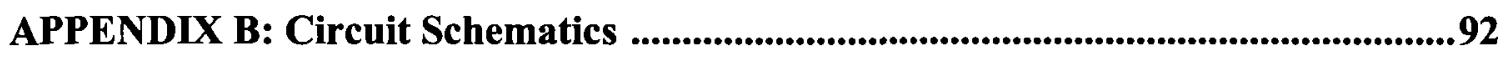

References .............................................................................................................................98 


\section{LIST OF FIGURES}

FIGURE 2.1 Triangulation Technique With Beacon Node For Central Control.............7

FIGURE 2.2 Non-Triangulation Technique For Individual Control ...........................7

FIGURE 2.3 Ultra Wideband Pulse ................................................................. 11

FIGURE 2.4 A Typical Mono-Gaussian Pulse And Its Power Spectrum ....................12

FIGURE 2.5 Spectrum Sharing Of Ultra Wideband Signal....................................15

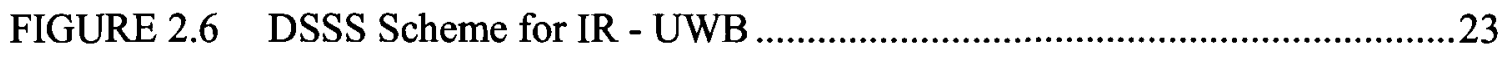

FIGURE 2.7 Multiband OFDM Power Spectrum .................................................24

FIGURE 2.8 Ultra Wideband Spectral Mask For Outdoor Communication ................25

FIGURE 2.9 Ultra Wideband Spectral Mask For Indoor Communication ...................26

FIGURE 2.10 Ultra Wideband Spectral Mask For Imaging Devices ..........................27

FIGURE 2.11 Ultra Wideband Spectral Mask For Vehicular Radar Systems................28

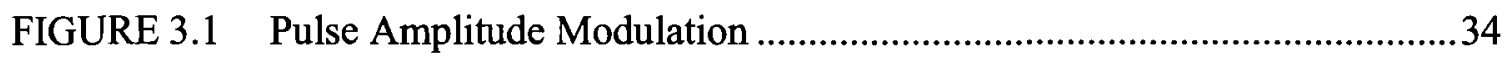

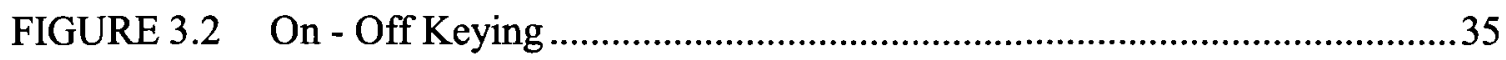

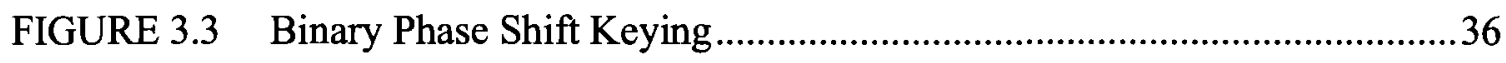

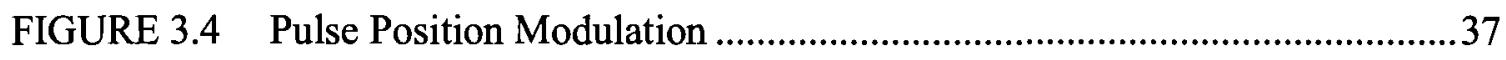

FIGURE 3.5 Transmitted Reference Modulation .............................................39

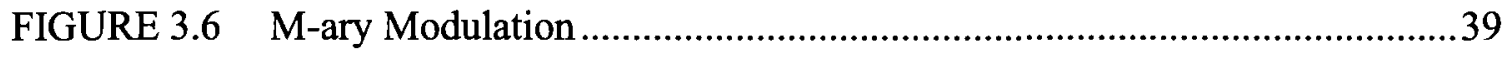

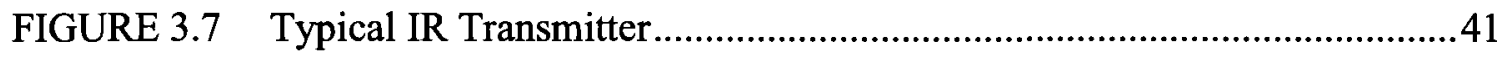

FIGURE 3.8 Typical OFDM Transmitter .........................................................42

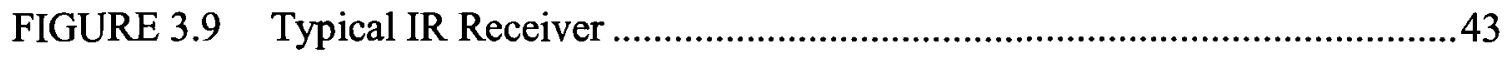

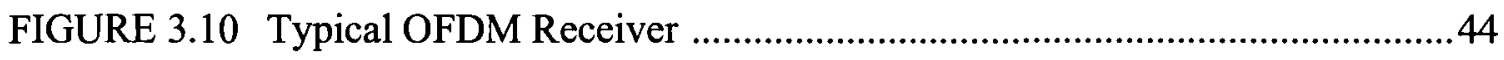


FIGURE 3.11 Serial Data Transceiver Interface For UWB Communication .56

FIGURE 4.1 UWB Channel Modeling In MATLAB ..........................................63

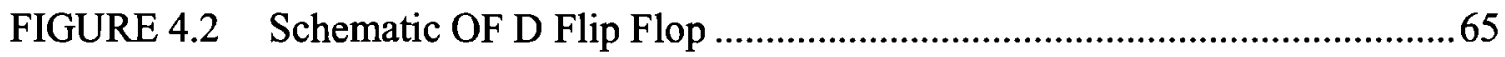

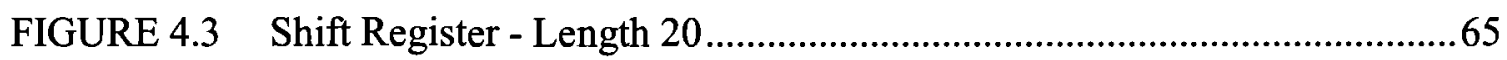

FIGURE 4.4 Data Sequence Through Shift Register ............................................66

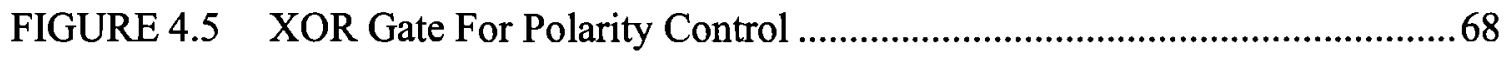

FIGURE 4.6 Three Tap Filter Shown With Pre, Main \& Post Tap Currents ................70

FIGURE 4.7 Transmitter Driver Consisting Of 18 Stages........................................71

FIGURE 4.8 Stacked Inductors For Wireless Communication .................................73

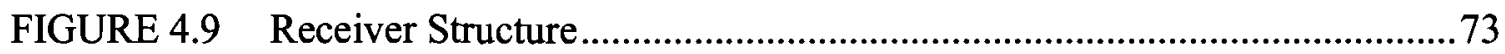

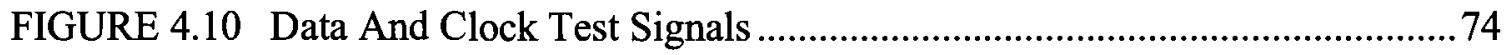

FIGURE 4.11 Transmitter Block Diagram .............................................................. 76

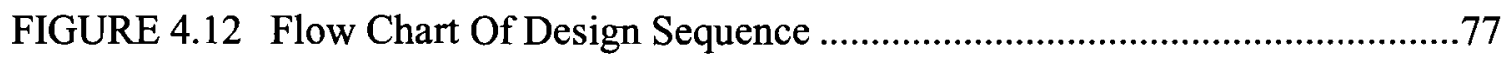

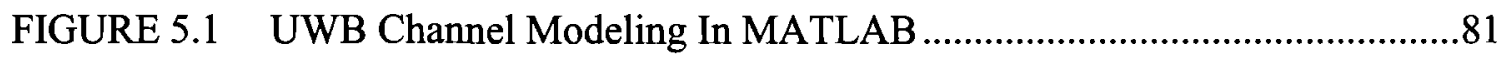

FIGURE 5.2 Generated Random Noise Signal ...............................................82

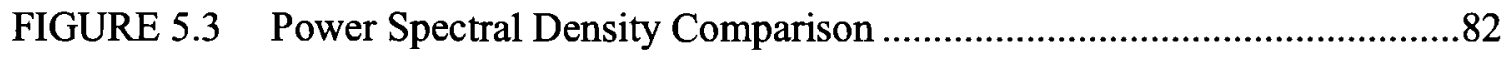

FIGURE 5.4 Transmitted Data Sequence .............................................................. 83

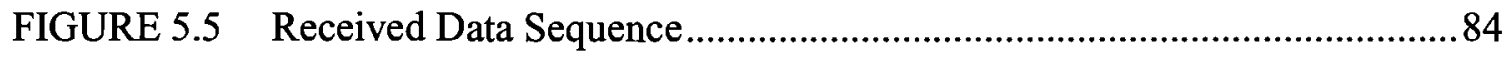

FIGURE 5.6 Comparison Between Transmitted And Received Data Sequences .........85

FIGURE 5.7 Power Spectrum of Transmitted Sequence in SPECTRE .......................86 


\section{LIST OF TABLES}

TABLE 2. 1 FCC Spectral Emission Limits For Indoor Communication Devices.......28

TABLE 2. 2 FCC Spectral Emission Limits For Outdoor Communication Devices....29

TABLE 2. 3 FCC Spectral Emission Limits For Imaging Systems ..........................29

TABLE 2. 4 FCC Spectral Emission Limits For Vehicular Radar Systems .................29

TABLE 3. 1 Comparison With other Reported Work ..............................................54

TABLE 4. 1 Shift register specifications ..........................................................66

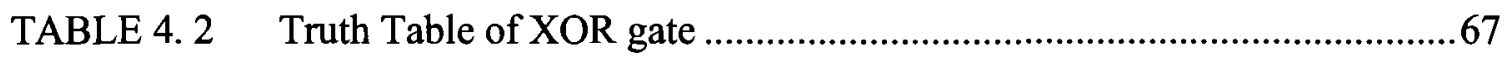

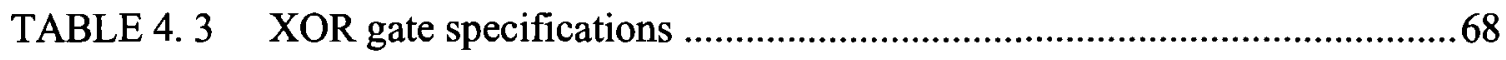

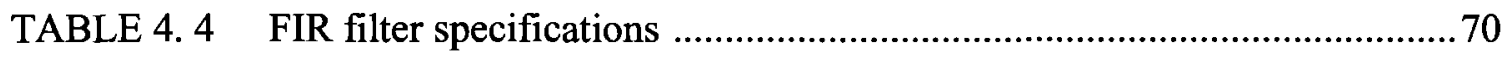

TABLE 4. 5 Total Power consumed in the transmitter .........................................78

TABLE 4. 6 Comparison With other Reported Work.............................................78 


\section{LIST OF SYMBOLS \& ABBREVIATIONS}

$\begin{array}{ll}\text { Ground } & \begin{array}{l}\text { Power Supply } \\ \text { NMOS }\end{array} \\ \text { Buffer } \\ \text { Inverter } \\ \text { Resistor } \\ \text { Inductor } \\ \text { Capacitor }\end{array}$




\section{ABBREVIATIONS}

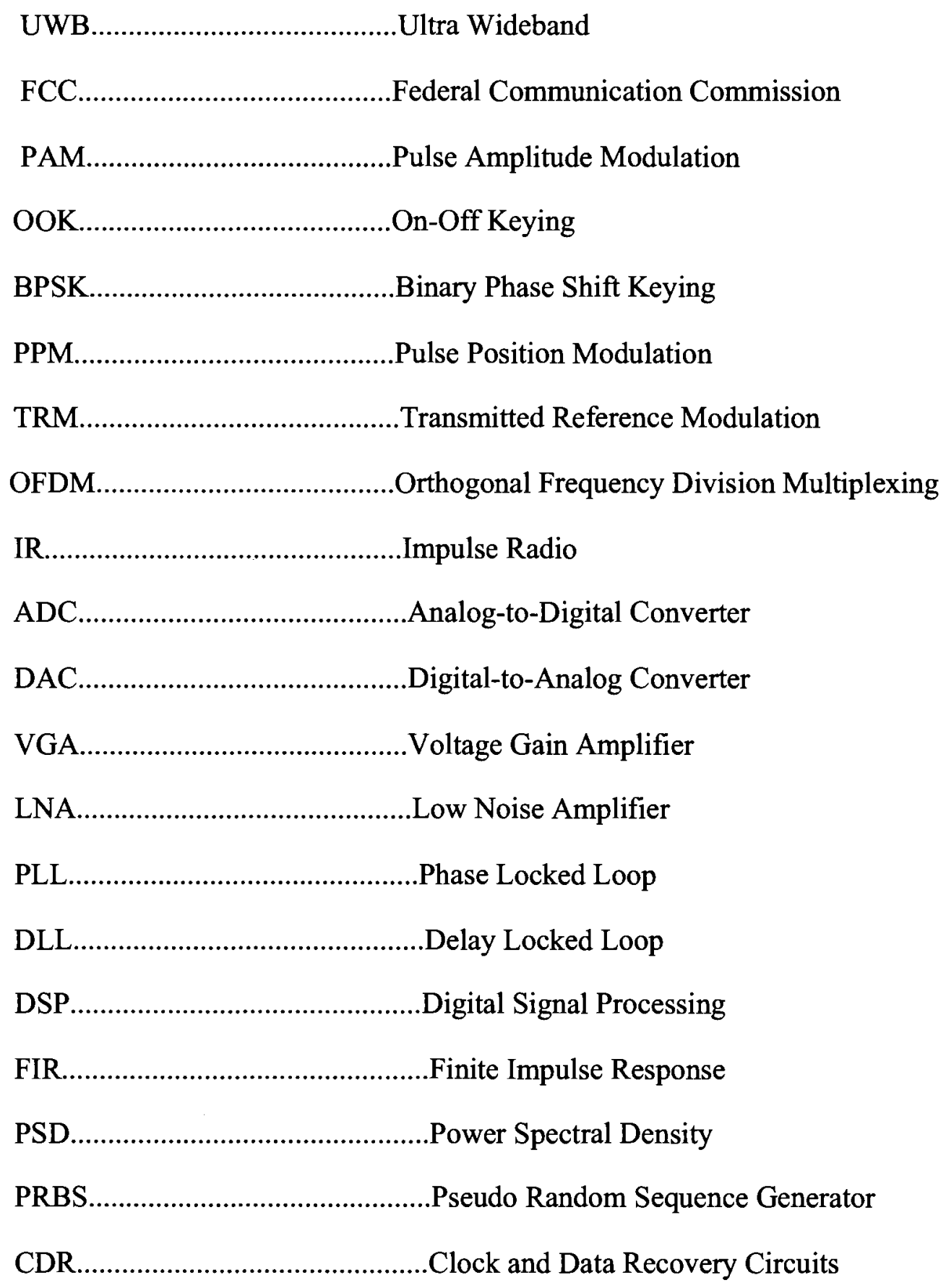




\subsection{Chapter Overview}

This chapter provides an introduction to the thesis work and discusses the motivation for the reported work. It concludes with a brief synopsis of thesis organization.

\subsection{Introduction}

The era of wireless communication devices has witnessed an incredible boost during the past decade. Till the last decade, battery powered portable devices capable of transmitting and receiving voice, text, and data messages operating at a limited bandwidth were considered the most sophisticated scientific invention. But with the increase in demand of wireless devices for transfer of any data ranging from fundamental to sensitive applications has forced the scientists to work not only on wider bandwidth but much more advanced wireless devices. Wireless devices are not only used for communicating the data but also for collection of data by deploying state-of-the-art sensor network systems. Now- 
adays, this is undoubtedly the most focused and worked upon area in wireless communication systems. Sensor network systems can be deployed for monitoring traffic situation on a highway or for military surveillance. Like all other wireless devices, the most important performance parameter for sensing devices is the power consumption. A fast connection or huge bandwidth is of no use if the devices can last for only a small period, unlike most of the other wireless devices which are rechargeable in case of power loss, the entire sensor network can go down if it is not designed to consume power efficiently. The most important component of a sensor network system is transceiver since data transfer requires more energy than sensing or processing. This is where UWB transceivers comes in the picture. Ultra wideband transceivers works on a huge bandwidth of $3.1-10.6 \mathrm{GHz}$ and they have an emission power limit of $-41 \mathrm{dBm} / \mathrm{MHz}$ which make them extremely low power and well-suited for use in sensor network systems. According to Federal Communication Committee or FCC [1] any wireless signal that covers the bandwidth of $500 \mathrm{MHz}$ or $20 \%$ fractional bandwidth is considered ultra wideband signal.

\subsection{Thesis Motivation}

There is a number of new developments in the area of low power and low complexity wireless communications. The motivation of the thesis was to explicitly visit the challenges that arises in the implementation of low power ultra wideband systems and wireless chip-to-chip communication. UWB systems uses low rate, ultra low power pulses for

UWB Ultra-Low-Power Short Distance FIR Spectrally Shaped NRZ Transmission 
data transfer and require complex modulation architecture to meet the spectral requirements. It is a challenging task to realize a system that is much higher rate, low power and simpler to implement yet compliant with the required power spectrum.

Wireless chip-to-chip communication is another evolving area which is considered as future of a system on board designs. A lot of work is being carried out to explore the hurdles that lies in the implementation of the lowest power consumption yet high speed communication technique.

In this work it is attempted to review existing design and hardware solutions and to present a viable solution that would meet as many of the above challenges as possible. In accordance with the focus of our research group, NRZ signaling has been chosen to demonstrate UWB communication. FIR shaping of a serial data bit stream is proposed and a feasibility of a wireless $10 \mathrm{Gbps}$ chip-to-chip communication is conducted. SERDES like digital output data stream transmission over a close proximity like chip-to-chip wireless link is demonstrated. The achieved energy per bit compares favorably with other reported transceivers.

\subsection{Thesis Organization}

The thesis is organized as follows:

Chapter 2 provides the literary background information about sensor network systems

UWB Ultra-Low-Power Short Distance FIR Spectrally Shaped NRZ Transmission 
and ultrawideband communication. The chapter comprehensively debates over various implementation issues that are associated with low power \& high performance ultra wideband transceivers and also lists the advantages and applications of ultra wideband transceivers in wireless communication.

Chapter 3 elucidate the design consideration issues associated with ultra wideband transceivers. Channel modeling and receiver implementation considerations are described in depth. The chapter concludes with a particularized summary of the reported work so far in the same area.

Chapter 4 provides the design implementation details and performance evaluation of the ultra wideband transmission / reception channel through transistor level realization. Basic building blocks and test bench setup are presented in detail and all of the simulation parameters are summarized in a table. This chapter concludes with the performance evaluation of the proposed circuit structure.

Chapter 5 furnishes the simulation results and concludes with an analysis of obtained results.

Chapter 6 derives the conclusion from the work and specifies the potential area for future contributions.

References are listed in the end. 


\subsection{Chapter Overview}

This chapter covers the background of sensor networks and ultra wideband communication.

\subsection{Introduction To Wireless Sensor Networks}

A wireless sensor network consists of two or more sensor nodes capable of collecting and exchanging information with each other. Each sensor node comprises of a cluster of sensors, microprocessor, transceiver, analog-to-digital converter (ADC), memory and power source. Wireless sensor networks covers vast application areas varying from as sensitive application as military surveillance to as fundamental as medical diagnostics and as habitual as environmental monitoring. 


\subsection{Characteristics Of Sensor Network}

This section discuss some of the general characteristics of the sensor networks that are being deployed today.

\subsubsection{Self-Organization}

A wireless sensor network consists of many nodes, each with multiple links connecting to other nodes. The sensed information is sent through a hopping route from the collection node to the processing node. Manually defining the routing path can be expensive and impractical. One of the most important characteristics of the recent sensor networks is self-organization. This characteristic allows the nodes to work on a store and update routing format thus enabling them to calculate the shortest routing path to the targeted node. The two main techniques are called Triangulation and Non-Triangulation Techniques. In triangulation operation, only a small number of nodes are deployed with a pre-configured position with respect to a global coordinate system. These nodes are called beacon nodes. Rest of the nodes learn and update their routing paths by taking the beacon nodes as reference nodes. In non-triangulation operation, there is no central control and each node uses specific position and distance calculation algorithms to create the effective routing path [2] and [3]. Figure 2.1 and Figure 2.2 shows the triangulation and non-triangulation techniques.

UWB Ultra-Low-Power Short Distance FIR Spectrally Shaped NRZ Transmission 


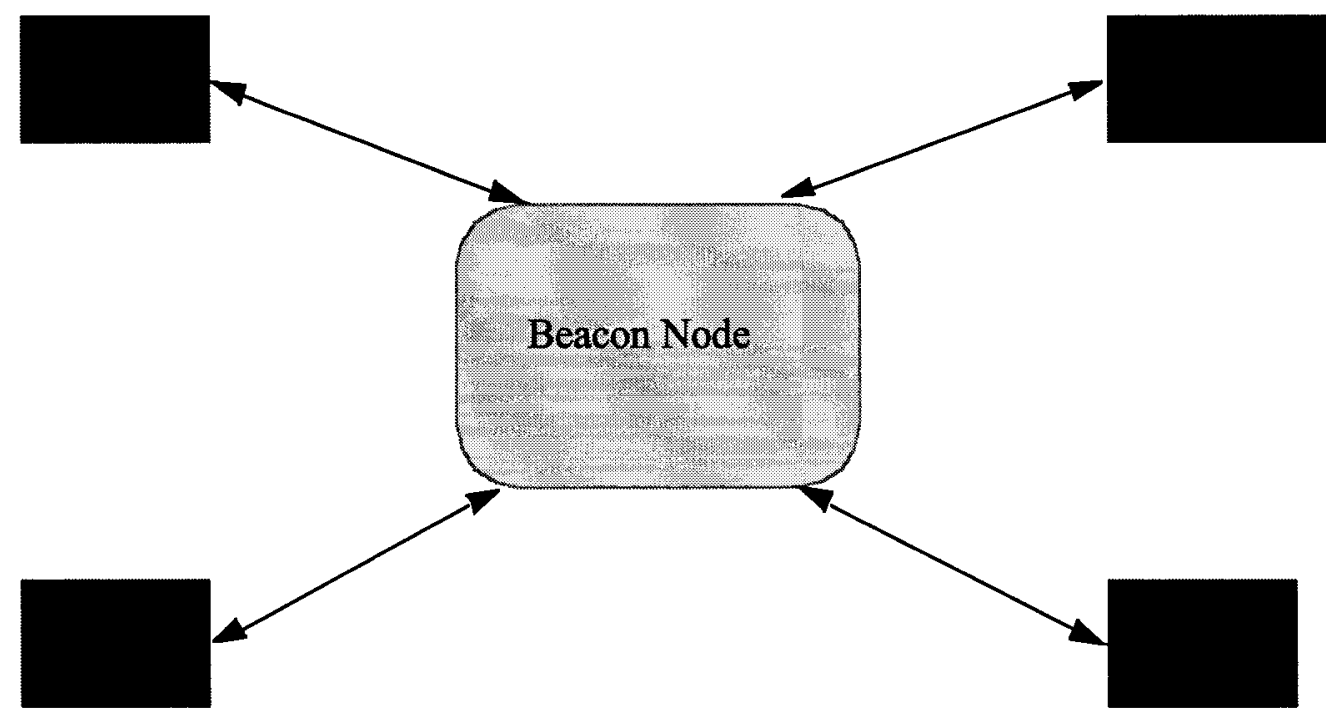

FIGURE 2.1 Triangulation Technique With Beacon Node For Central Control

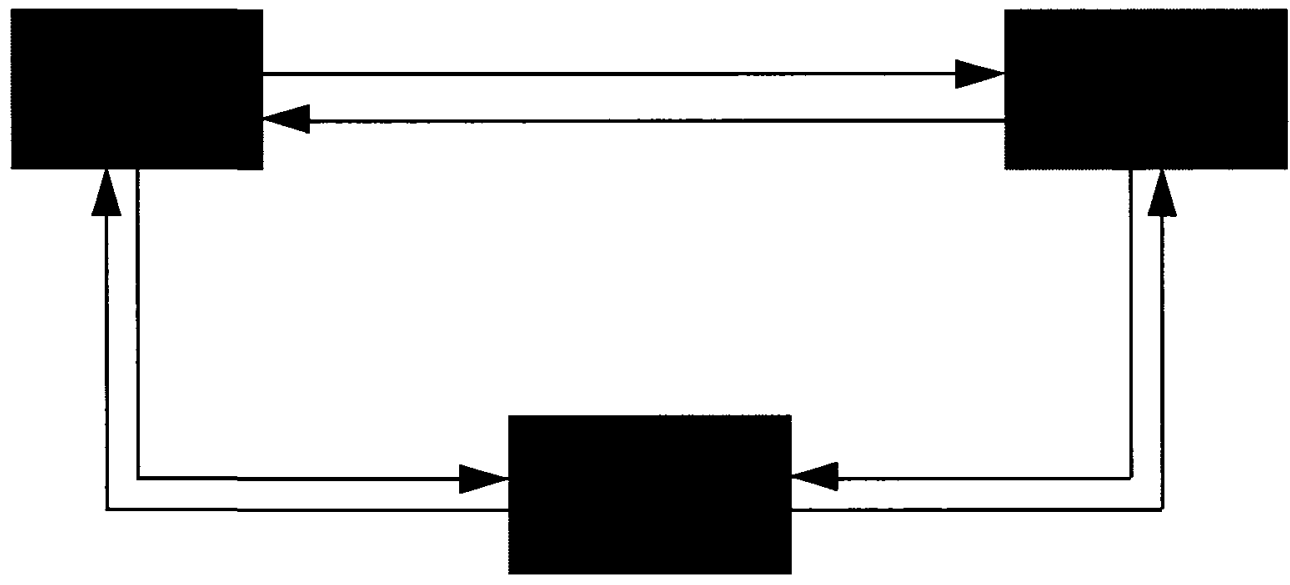

FIGURE 2.2 Non-Triangulation Technique For Individual Control 


\subsubsection{Self-Shutdown}

Several network nodes may remain idle for a long period of time with no data to receive and send, self-shutdown allows them to save their available energy resources, whether in the form of batteries or energy-harvesting. However, large variations in the operating status of nodes creates significant variations in connectivity and thus makes the network more vulnerable to harsh environmental conditions.

\subsection{Implementation Challenges}

Deployment of a sensor network faces many implementation challenges. Most critical among them are lower power consumption and efficient algorithmic realization for localization and communication between nodes.

\subsubsection{Algorithmic}

Proficient routing techniques, data exchange protocols and at-node processing capabilities forms the backbone of a sensor network. Concentrated work is being done in these areas for improved performance. Localization techniques enables each node to acquire its position in a sensor network and ensures the optimum routing path existence for data exchange. At-node processing is also important since the most power consuming operation in a sensor network is information exchange therefore, instead of transferring raw 
data, certain processing of data took place at the node before it is send forward to the destination node.

\subsubsection{Energy}

Energy conservation is no doubt the most important feature of a WSN. The average power consumption of a sensor node must be between $10-100 \mu \mathrm{W}$ with a single $1.5 \mathrm{~V}$ AA alkaline battery to last the node for a lifetime of 1-5 years [4]. Communication is the most power consuming operation that a sensor node performs. Sensor networks minimizes communication between nodes by several methods. Mostly used methods are turning off the radio when no communication is necessary and local processing of data. For example the sensor node can collect the information and only communicate when they detect an alien pattern. However, this approach can only be adopted in intelligent sensor networks. Another way is to employ low power sensors that will trigger high power sensors after collecting a set of data for a specific application for example the environmental conditions of specific area can be monitored for two hours and the results are recorded in a table by the low power sensor. The low power sensor then triggers the high power sensor and passes the complete recorded summary which is then processed at high power sensors. Finally compression and scheduling can also help to reduce power consumption in sensor networks. The radios go up according to a pre-scheduled time and the data can then be transmitted. The radio can then shut-off after a specific timing duration and ignores the unnecessary packets. 
Different applications uses different combination of the above mention techniques since some of them may be conflicting in nature. Wireless sensor network systems require low power implementations and most of the wireless standards does not comply with this criteria. However, since UWB communication systems has to follow a strict power emission limit, it is indeed the most promising approach for successful implementation of low power sensor networks.

\subsection{Introduction To Ultra Wideband Communication}

Ultra wideband technology enables information exchange over a large bandwidth at the same time allowing it to coexist with current radio system with minimal or no interference. According to the modern definition, any wireless communication technology that produces signals with a bandwidth wider than $500 \mathrm{MHz}$ or a fractional bandwidth greater than $20 \%$ can be considered as UWB. Ultra wideband technology in the beginning was only used for military application in the form of impulse radars.

As interest in the commercialization of UWB has increased over the past several years, developers of UWB systems began pressuring the FCC to approve UWB for commercial use. In February 2002, the FCC approved the First Report and Order (R\&O) for commercial use of UWB technology under strict power emission limits of $-41 \mathrm{dBm} / \mathrm{MHz}$ for various devices.

UWB Ultra-Low-Power Short Distance FIR Spectrally Shaped NRZ Transmission 


\subsubsection{Basic Concept}

Impulse radio based ultra wideband communication is the most commonly adopted technique for low power operations. The impulse radio based technique uses short duration ultra wideband pulses for transfer of data with very small duty cycle. Duty cycle can be defined as the ratio between the time when the pulse is present to total transmission time. Equation 2.1 presents this relationship which is further explained with the help of Figure $2.3[5]$.

$$
D=\frac{T_{O N}}{T_{O N}+T_{O F F}}
$$

Where Duty Cycle

$T_{O N}=$ The time when pulse is present in nsec

and $\quad T_{O F F}=$ The total transmission time in nsec

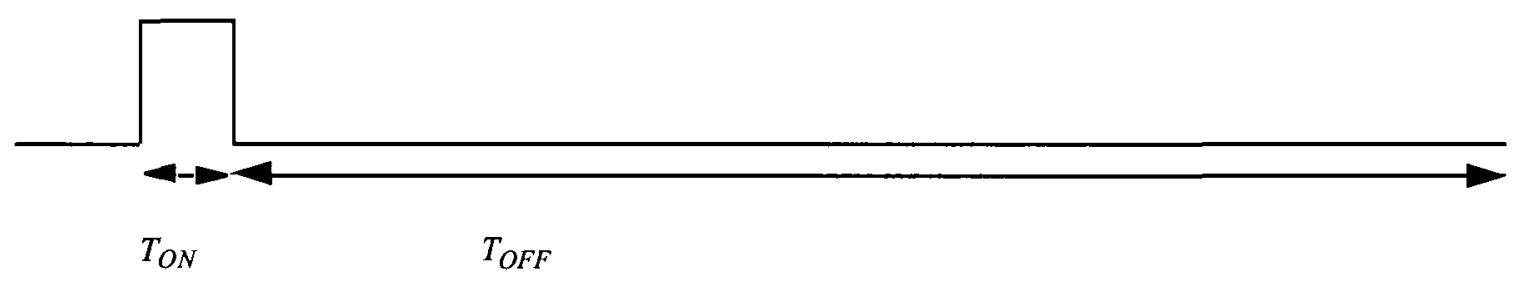

FIGURE 2.3 Ultra Wideband Pulse

UWB Ultra-Low-Power Short Distance FIR Spectrally Shaped NRZ Transmission 
As the pulse is present for a very small duration, it decreases their average power quite significantly. Since frequency is inversely related to time, the power is distributed along a wide bandwidth in the order of several gigahertz $(\mathrm{GHz})$ resulting in very low power spectral density (PSD). Figure 2.4 shows the UWB pulses in time domain and frequency domain.

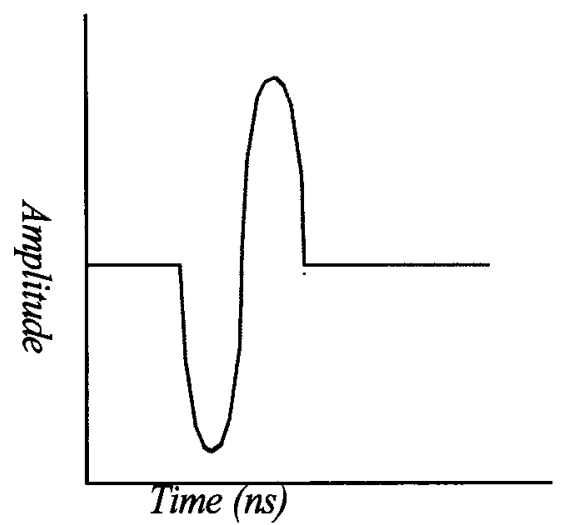

(A)

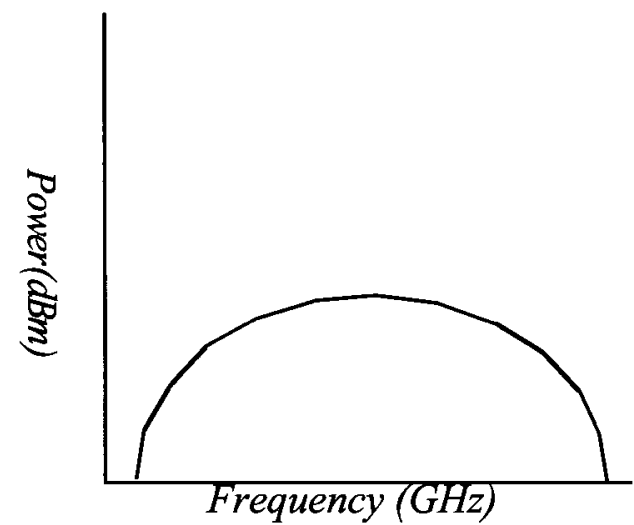

(B)

FIGURE 2.4 A Typical Mono-Gaussian Pulse And Its Power Spectrum 


\subsubsection{UWB Signals}

According to FCC, UWB signals must have bandwidths of greater than $500 \mathrm{MHz}$ or a fractional bandwidth larger than 20 percent at all times of transmission. Fractional bandwidth is defined by the ratio of bandwidth at $-10 \mathrm{~dB}$ points to center frequency [1].

$$
B_{F}=\frac{F_{H}-F_{L}}{F_{C}} \times 100 \%
$$

Where,

$$
\begin{aligned}
& B_{F}=\text { Fractional Bandwidth } \\
& F_{H}=\text { Higher cut-off frequency } \\
& F_{L}=\text { Lower cur-off frequency } \\
& F_{C}=\text { Centre frequency }
\end{aligned}
$$

and

\subsection{Advantages Of Ultra Wideband Communication Systems}

The deployment of ultra wideband system is a challenging task due to some of the major limitations that are discussed in next section, however, it is an evolving area for wireless communication systems. This section discusses, the advantages of using ultra wideband for wireless communications [5] and [6]. 


\subsubsection{Frequency Spectrum Sharing}

The emission power requirement for an ultra wideband is substantially low, that is, -41.3 $\mathrm{dBm} / \mathrm{MHz}$ as prescribed by the FCC. This power constraint has given UWB a unique advantage which is its ability to share the frequency spectrum with other standards. Due to such lower power limitation UWB signal resides below the noise level of a typical narrowband or wideband signal. This co-existence occurs with almost no interference from UWB signals in the frequency spectrum. Figure 2.5 shows this concept of spectrum sharing of an ultra wideband signal with narrowband and wideband signals.

\subsubsection{Robustness Against Eavesdropping}

One of the characteristic property of UWB technology is the safety associated with the transmission because of low power limitation. These signals are almost immune to detection and interception, for the unauthorized detection of such a low power signal is not possible from large distance. UWB signals are pre-coded according to a pattern specific to the transmitter / receiver pair, matched filters are used for regenerating the signal at the receiver end. Hence, even if the signal is captured and not discarded as noise, it is almost impossible to decode the information hidden in it in the absence of some pattern matching technique. This property makes UWB a highly secure network and it is one of the prime reason why UWB is extensively used for military operations.

UWB Ultra-Low-Power Short Distance FIR Spectrally Shaped NRZ Transmission 


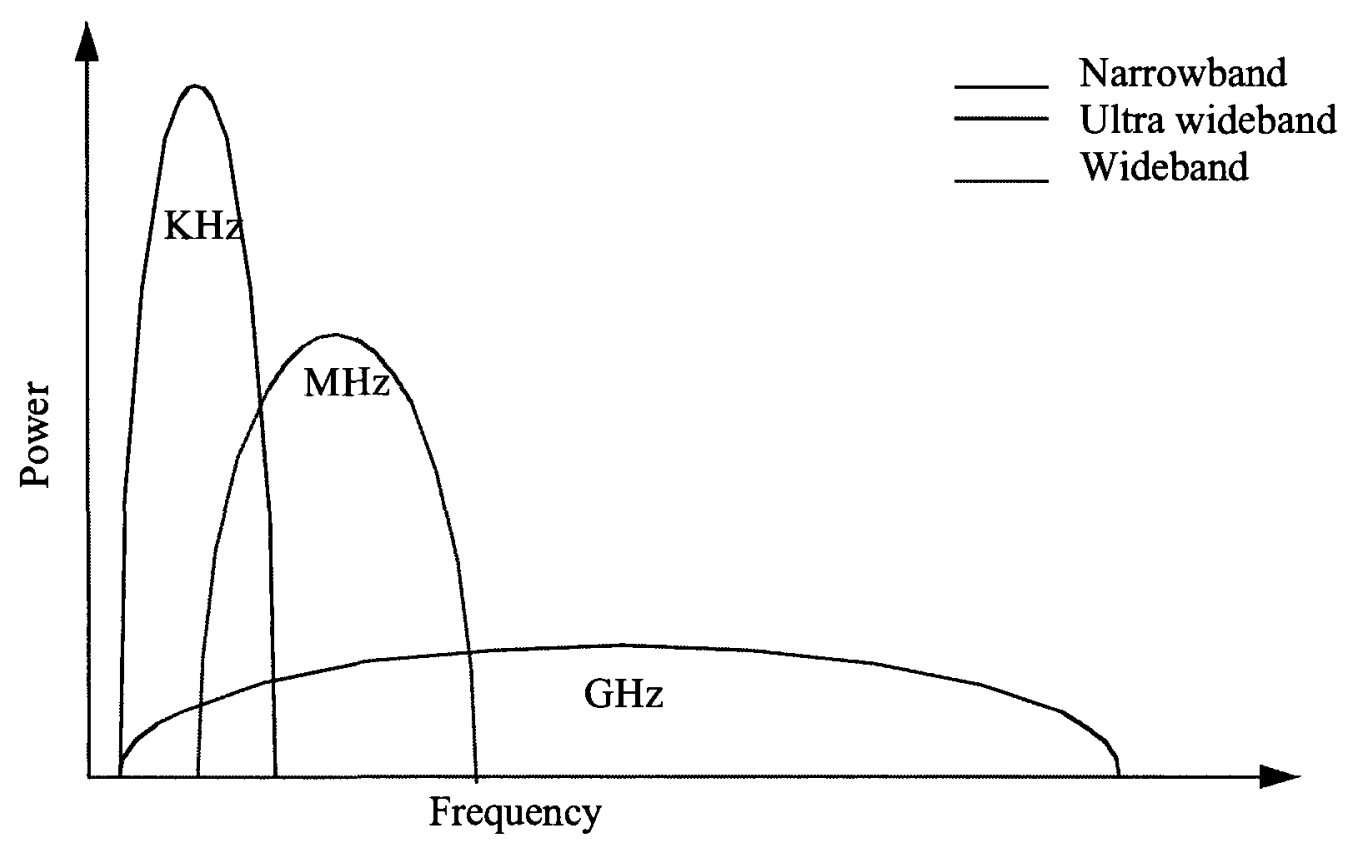

FIGURE 2.5 Spectrum Sharing Of UItra Wideband Signal

\subsubsection{Penetration Properties}

UWB signals are capable of penetrating through walls and different materials. The low frequency component of the UWB frequency spectrum have longer wavelengths and thus are capable to penetrate through walls or ground.

\subsubsection{Robustness Against Multipath Fading}

The high temporal resolution of UWB signals make them somewhat immune to multipath fading. Since the pulses are of really short duration, it is highly unlikely that they will reflect and distort the line-of-sight signals. However, multipath fading is of significant 
importance in indoor channels where the scattering surfaces are closer and can extensively distort the signal.

\subsubsection{Flexibility}

UWB technology offers great flexibility in terms of optimizing different performance parameters such as data rate, range, power and quality. Data rate is defined as the maximum amount of data that can be transmitted per second over a channel. According to the Hartley-Shannon's formula [25]:

$$
C=B \log (1+S N R)
$$

$$
\begin{aligned}
& \text { Where, } \\
& \qquad \begin{array}{l}
C=\text { Channel Capacity or Data Rate } \\
B=\text { Bandwidth of the Channel } \\
\text { and }
\end{array}
\end{aligned}
$$

As it is evident from the above formula that the increased bandwidth results in higher data rate for the UWB system of the order of several gigabits per second (Gbps). Due to FCC limitation of low transmission power such a high data rate is possible over short range. SNR is bandwidth dependent but it is possible to keep the transmitted power under limitation by transmitting over short distances. Similarly, data rate and range can be traded of for power, especially for low data rate and short range applications. 


\subsubsection{Robustness Against Channels}

Data rate is logarithmically dependent on SNR as evident from equation 2.3. Therefore, UWB systems are capable of providing a high data rate even in communication channels with relatively low SNR.

\subsubsection{High Processing Gain}

Processing gain of a system is a measure of the system's resistance to jamming. Since UWB system operates over a range of frequencies, it is very difficult to jam every frequency in the band. Processing gain is a signal to jammer ratio where jamming is achieved by deliberate interference to the signal.

\subsection{Implementation Challenges}

Although UWB systems offer unique advantages for wireless communication, its actual implementation is somewhat challenging. There are several fundamental and practical issues that need to be resolved to ensure the success of this technology in the wireless communication market. This section discusses the implementation issues that UWB system faces today. 


\subsubsection{Pulse Shape Distortion}

The distortion of the pulse-shape while passing through the transmission link is one of the most fundamental challenges that lies in the implementation of UWB systems. This distortion can be mathematically shown by the Friis transmission formula [5]:

$$
P_{R}=P_{T} \times G_{T} \times G_{R}\left(\frac{C}{4 \times P_{i} \times D \times f}\right)^{2}
$$

Where, $\quad P_{R}=$ Received Signal Power

$$
\begin{aligned}
& P_{T}=\text { Transmitted Signal Power } \\
& G_{T}=\text { Transmitter Antenna Gain } \\
& G_{R}=\text { Receiver Antenna Gain } \\
& C=\text { Speed of Light } \\
& D=\text { Distance between transmitter and receiver }
\end{aligned}
$$

and $\quad f=$ Signal frequency

As evident from equation 2.3 , the receiver power will decrease proportionally with the increase in frequency. Since the narrow band signals occupy a very small frequency range therefore, the change in signal power with the change of frequency is significantly lesser than UWB signals and can be overlooked. However, UWB pulses are much more low powered and weak and thus can be drastically distorted with the reduced receiver power. Since most UWB-IR receiver uses correlation techniques to identify the pulses and 
retrieve information, the reception of distorted pulses minimizes the chances of pulse detection and hence results in low throughput of the system. Here, it should be noted that the above relationship is for ideal cases with constant antenna gain for both transmission and reception purposes. UWB antenna are designed specifically for wide impedance bandwidth covering the bandwidth where majority of source pulse energy falls in and constant gain at desired directions. Reference [39] discusses the UWB antenna design issues.

However, the practical factors such as impedance mismatch, reflection, absorption along the channel and misalignment or polarization effects contribute to the pulse shape distortion and can be added to the simplified relation above. For strong multipath effects and NLOS channels further modifications are done to the above relationship with gain of antennas taken as of mean effective value.

\subsubsection{Channel Estimation}

Channel estimation is critical for UWB systems since most receivers uses the correlation technique to match the received signal with a pre defined template so it is necessary to estimate the unpredictable behavior of the channel such as the delay, distortion, coefficients and attenuation associated with the transmission path but because of high frequency range the received pulses can be considerably distorted and hence channel estimation becomes very complex.

UWB Ultra-Low-Power Short Distance FIR Spectrally Shaped NRZ Transmission 


\subsubsection{Accurate Synchronization}

Accurate synchronization of the receiver to extremely narrow pulses is yet another challenge that the UWB technology faces. Such short duration pulses of the order of ns makes the system all the more vulnerable to the timing errors such as jitter and drift.

Hence, excellent synchronization between the transmitter/receiver pair is essential for UWB communication.

\subsubsection{High Sampling Rate For Digital Implementation}

With such short duration pulses, very fast (of the order of GHz) Analog-to-Digital Converters (ADCs) are required to facilitate the operation of digital back-end. Using such high sampling rate converter means introducing a significant boast in power consumption and overall cost of the system which may provide hurdle in the use of UWB systems for power efficient and low cost applications such as sensor networks.

\subsubsection{Multiple-Access Interference}

Multiple-Access Systems are those systems where multiple users send information over the same transmission medium at the same time. At the receiver end, the received information is detected, separated and send forward to the user of interest. The interference between the multiple-access information or (MAI) is crucial for UWB signals. Since the

UWB Ultra-Low-Power Short Distance FIR Spectrally Shaped NRZ Transmission 
pulses are so low powered, and already corrupted by channel noise they are severely distorted by MAI, which makes it next to impossible to detect and forward the information appropriately to the user.

\subsubsection{High Performance Transceivers}

It is important that the UWB transceivers should have high performance parameters which includes low power, simple and practical transmitters as well as receivers having powerful processing capability and coherent digital structure.

\subsection{Single band vs Multi-band}

From the 1960 s to the 1990 s UWB technology was restricted to military and Department of Defence (DoD) applications for classified military operation. However, recently Federal Communication Commission (FCC) has allowed unlicensed used of $7.5 \mathrm{GHz}$ of frequency spectrum from $3.1 \mathrm{GHz}$ to $10.6 \mathrm{GHz}$ for UWB communication under the restriction that the transmission power should not exceed $-41.3 \mathrm{dBm} / \mathrm{MHz}$.

UWB emerged as a single band or Impulse Radio (IR) based technology, where information is sent in the form of nanosecond pulses which immediately acquire the whole bandwidth of $7.5 \mathrm{GHz}$. Until February 2002, the UWB term was specifically used in the context of impulse radio modulation. In February 2002, FCC released an amendment (Part 15) [1] that specifies the rules of UWB transmission / reception. According to this release, 
any signal with fractional bandwidth greater than $20 \%$ of the signal or having a bandwidth greater than $500 \mathrm{MHz}$ is considered as an Ultra Wideband signal.

FCC also declared the indoor and outdoor spectral masks restricting the transmission power such that UWB signals do not cause interference with narrowband signals also operating in the same frequency bands. This means that specifically any communication signal which has a bandwidth greater than $500 \mathrm{MHz}$ and obeys the power limitations is an UWB signal. This changed the whole scenario giving rise to the multiband approach.

The concept of multiband OFDM based UWB communication systems is that the entire bandwidth of 3.1-10.6 GHz is divided into $528 \mathrm{MHz}$ bands and employs OFDM in each band. Each band consists of 128 sub channels of $4.125 \mathrm{MHz}$ [7]. The use of OFDM eliminates ISI problem due to multipath, eliminating the need of a complex equalizer.

However, OFDM approach also require relatively large computational power due to Fast Frequency Transform (FFT) [8]. The following section discuss the two candidates for the 802.15.3a standard: Direct-Sequence and Multiband OFDM.

\subsubsection{Direct-Sequence UWB}

Direct Sequence UWB is the single band time-domain signal processing approach that uses the nanosecond pulses combined with the direct sequence spread spectrum (DSSS) techniques for information exchange. Each data symbol is spread into a number of chips using specific spreading code. The advantage of DS-UWB is the propagation of UWB 
pulses which experience no Rayleigh fading. The disadvantage is that inter symbol interference (ISI) can severely degrade the performance of DS-UWB systems. Figure 2.6 shows the concept of DS-UWB pulses.

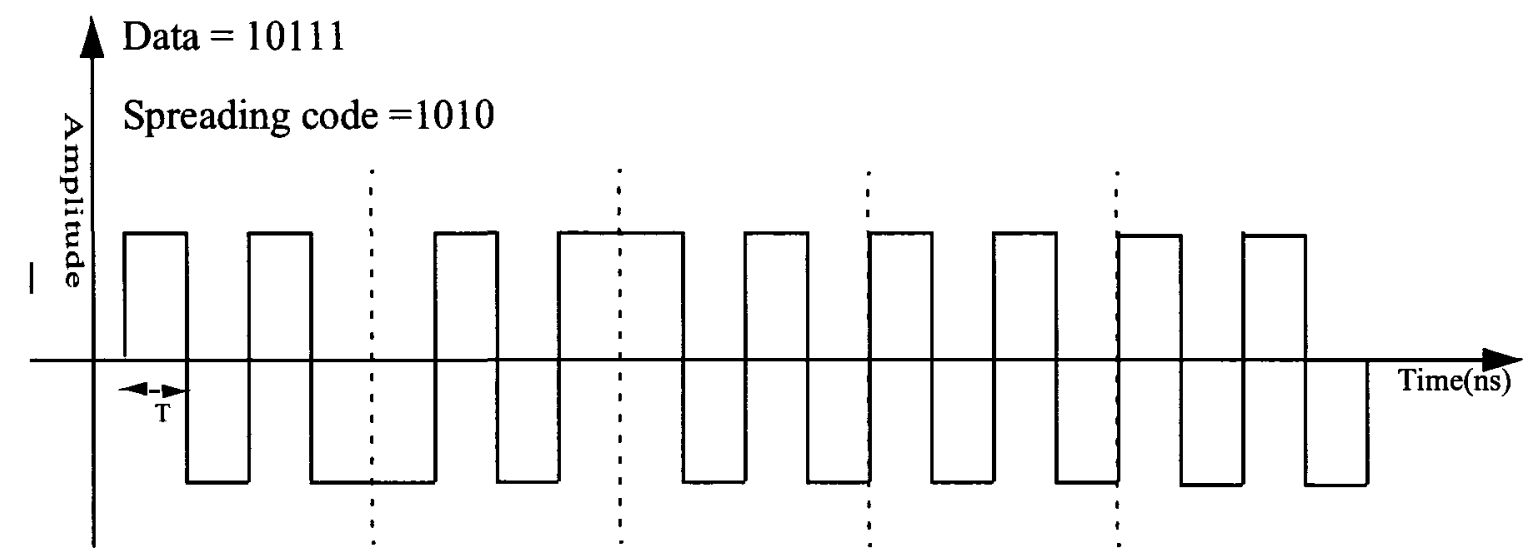

FIGURE 2.6 DSSS Scheme for IR - UWB

\subsubsection{Multi-band OFDM}

The fundamental idea behind the multi-band scheme is to split the entire bandwidth of 7.5 $\mathrm{GHz}$ into multiple frequency bands and efficiently utilizing the entire UWB spectrum by transmitting multiple signal at different frequencies. In the multi-band approach, the pulses are not as narrow as the traditional UWB pulses hence organization is easier. Below is the concept of Multiband OFDM UWB communication. 


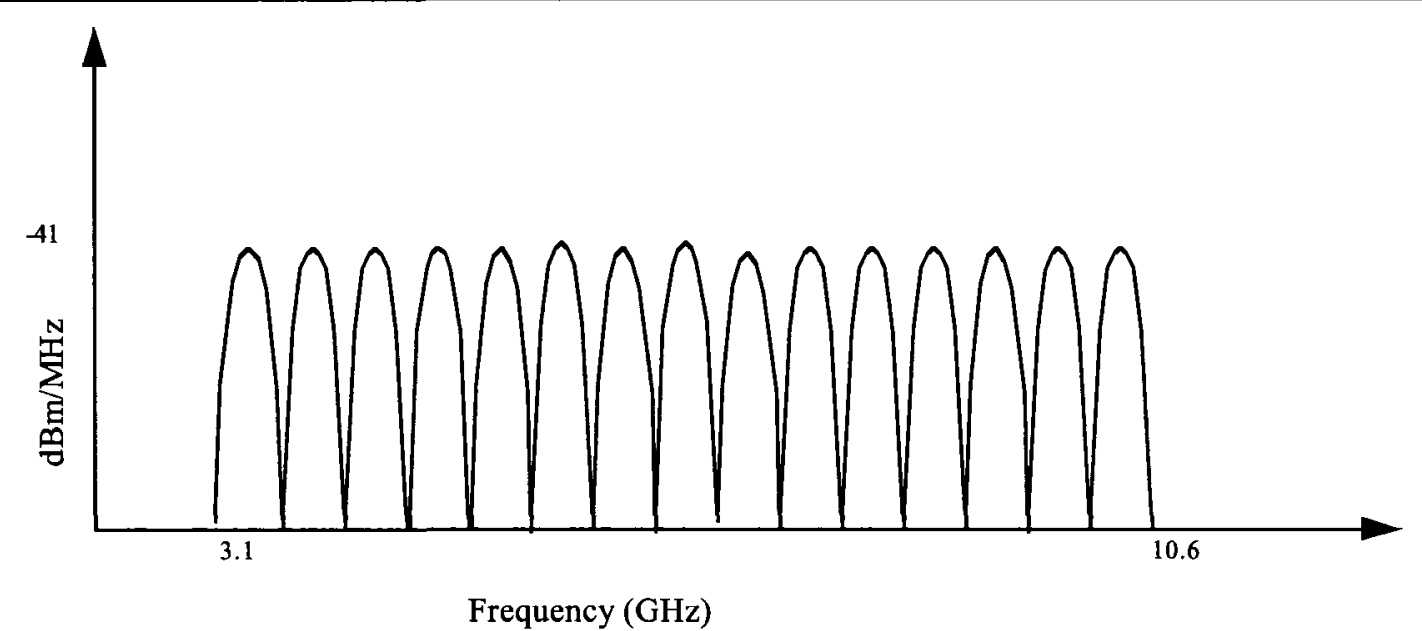

FIGURE 2.7 Multiband OFDM Power Spectrum

\subsection{FCC Emission Limits}

This section discuss the FCC emission limits for UWB devices. Based on FCC regulations, UWB devices are classified into three major categories: communications, imaging and vehicular radar.

\subsubsection{Communication Devices}

The spectral mask for outdoor and indoor communication devices as released by FCC is slightly different as the emission limit for the outdoor devices is $10 \mathrm{db}$ lower than that of an indoor device between 1.6 and $3.1 \mathrm{GHz}$. The figure below shows the two different spectral masks for indoor and outdoor devices. The PSD is expressed in terms of EIRP 
since it allows comparison between different types of emitters regardless of their shapes and sizes and if the antenna gain and link loses are known, it is possible to calculate the real transmitted power.

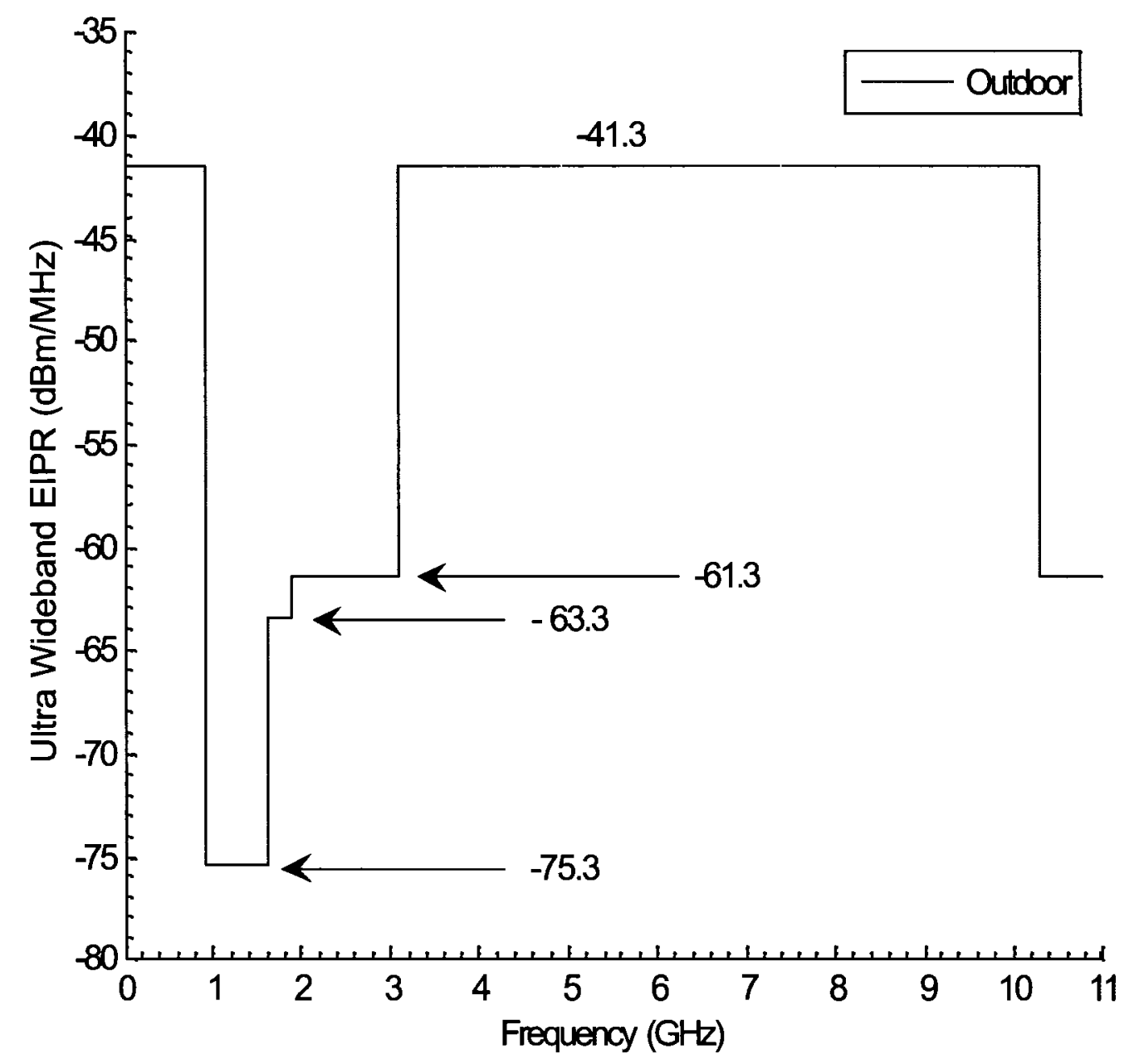

FIGURE 2.8 Ultra Wideband Spectral Mask For Outdoor Communication 


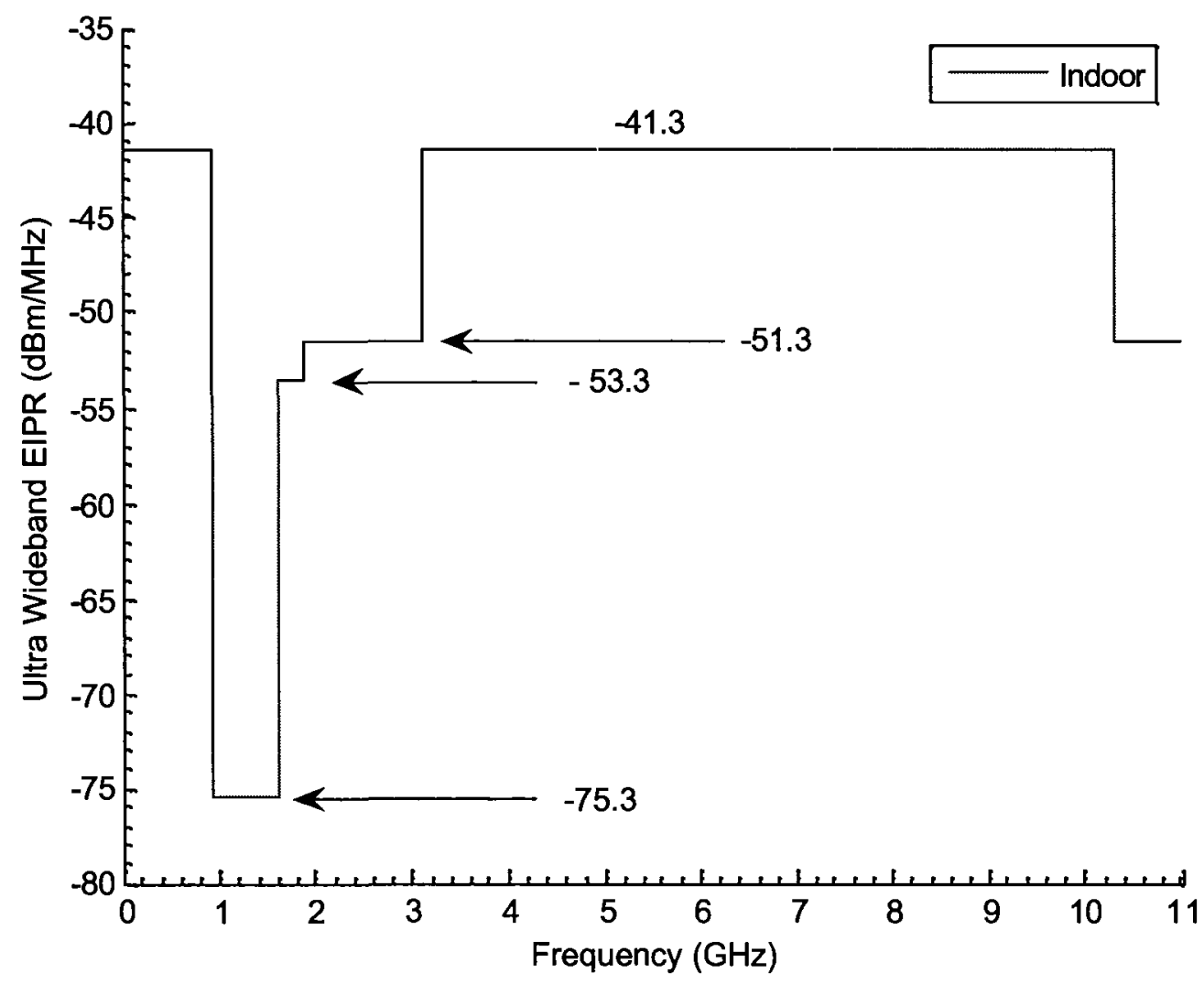

FIGURE 2.9 Ultra Wideband Spectral Mask For Indoor Communication

\subsubsection{Imaging Devices}

Imaging devices have three different spectral masks. The first one is for systems working below $960 \mathrm{MHz}$. The second one is for the imaging devices working above $1.9 \mathrm{GHz}$. The third one is for the imaging systems above $3.1 \mathrm{GHz}$. This spectral mask is shown in figure 2.10 whereas the detailed description of the FCC limitations of the other two systems can be found in Appendix A. 


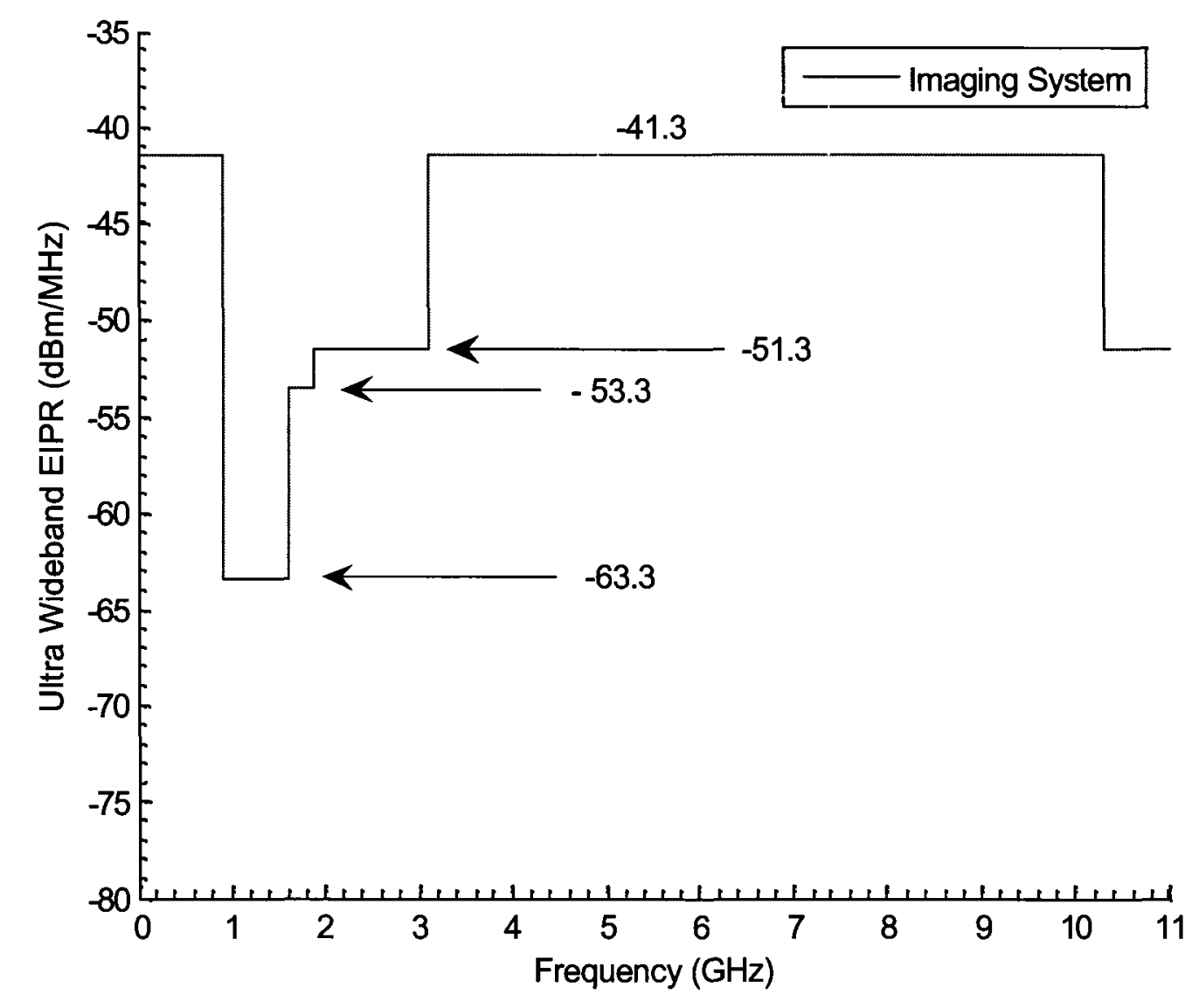

FIGURE 2.10 Ultra Wideband Spectral Mask For Imaging Devices

\subsubsection{Vehicular Radar System}

Vehicular radar system has an entirely different spectral mask as compared to communication devices and imaging devices. The transmission power limitation of $-41.3 \mathrm{dBm} /$ $\mathrm{MHz}$ is applicable to radar systems in the range of $22 \mathrm{GHz}$ to $29 \mathrm{GHz}$ which is much higher frequency range than that used for communication systems and imaging devices. The spectral mask is shown below: 


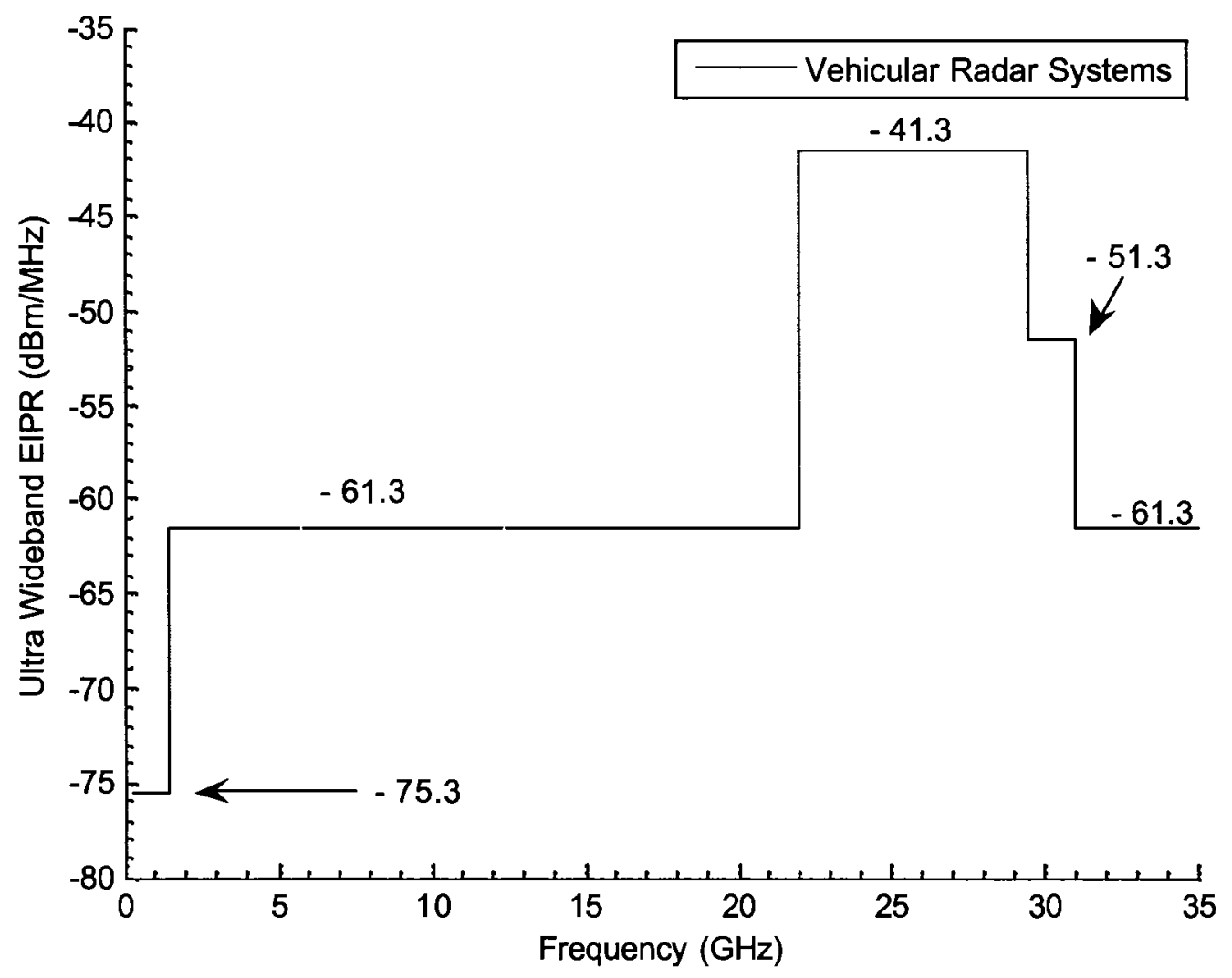

FIGURE 2.11 Ultra Wideband Spectral Mask For Vehicular Radar Systems

The frequency specifications for the above plotted graphs are listed in the tables below:

TABLE 2. 1 FCC Spectral Emission Limits For Indoor Communication Devices

\begin{tabular}{||c|c|}
\hline Frequency $(\mathrm{GHz})$ & E.I.R.P $(\mathrm{dBm} / \mathrm{MHz})$ \\
\hline $0.96-1.6$ & -75.3 \\
\hline $1.6-1.99$ & -53.3 \\
\hline $1.99-3.1$ & -51.3 \\
\hline $3.1-10.6$ & -41.3 \\
\hline Above 10.6 & -51.3 \\
\hline
\end{tabular}


TABLE 2. 2 FCC Spectral Emission Limits For Outdoor Communication Devices

\begin{tabular}{|c|c|}
\hline Frequency $(\mathrm{GHz})$ & E.I.R.P $(\mathrm{dBm} / \mathrm{MHz})$ \\
\hline $0.96-1.6$ & -75.3 \\
\hline $1.6-1.99$ & -63.3 \\
\hline $1.99-3.1$ & -61.3 \\
\hline $3.1-10.6$ & -41.3 \\
\hline Above 10.6 & -61.3 \\
\hline
\end{tabular}

TABLE 2. 3 FCC Spectral Emission Limits For Imaging Systems

\begin{tabular}{|c|c|}
\hline Frequency $(\mathrm{GHz})$ & E.I.R.P $(\mathrm{dBm} / \mathrm{MHz})$ \\
\hline $0.96-1.6$ & -63.3 \\
\hline $1.6-1.99$ & -53.3 \\
\hline $1.99-3.1$ & -51.3 \\
\hline $3.1-10.6$ & -41.3 \\
\hline Above 10.6 & -51.3 \\
\hline
\end{tabular}

TABLE 2. 4 FCC Spectral Emission Limits For Vehicular Radar Systems

\begin{tabular}{|c|c|}
\hline Frequency $(\mathrm{GHz})$ & E.I.R.P $(\mathrm{dBm} / \mathrm{MHz})$ \\
\hline $0.96-1.61$ & -75.3 \\
\hline $1.6-22$ & -61.3 \\
\hline $22-29$ & -41.3 \\
\hline $29-31$ & -51.3 \\
\hline Above 31 & -61.3 \\
\hline
\end{tabular}




\subsection{Applications Of Ultra Wideband}

UWB has fundamental applications in the field of communication systems, imaging systems and vehicular radar systems.

\subsubsection{Radar Systems}

The most powerful application of UWB system is radars. The ultra wide frequency spectrum enables the signal to penetrate various surfaces which makes UWB based ground penetrating radar (GPR) significant form military operations and also for detection of survivors under the rubble in case of earthquakes. Commercially, also same radars can be used on construction sites to detect any electrical or plumbing faults. The precise and accurate time resolution can be used in vehicular radar system for avoiding collisions and providing road side assistance.

\subsubsection{Imaging Systems}

The most fundamental application of UWB imaging system is in the field of medicine. This enables remote monitoring of transplanted or operated organs. Similarly, through wall imaging and area surveillance are critical not only for military operations but also for civilian usage in the form of home security where these imaging system can detect the presence of a human inside the house. Similarly, it can be useful for firefighters in their rescue operations.

UWB Ultra-Low-Power Short Distance FIR Spectrally Shaped NRZ Transmission 


\subsubsection{Communication Systems}

The extensive use of UWB technology in the communication sector can never be overlooked. Because of wide bandwidth, low power consumption and high data transfer rate UWB finds application in a wide area ranging from wireless home networking, UWB wireless mouse, keyboard, wireless speakers, wireless USB, and many more consumer electrical products.

\subsubsection{Wireless Sensor Network Systems}

One extensively emerging area of UWB application is its usage for deployment of sensor network systems. The sensor network systems are crucial for military application such as location monitoring and enemy tracking in battlefields and on country borders. Extended lifetime of sensor networks require low power consumption and hence UWB low power transceivers are excellent candidate for such applications. The sensor networks are also used for habitat monitoring, environmental monitoring and home automations.

The idea of the thesis work evolved from this specific application of UWB systems. Sensor networks works on low data rate and low power consumption however the energy per bit is quite high due to the low data rate. The temptation of the work was to create a low power system with high data rate so as to achieve low energy per bit and then identify how efficiently such a system can be used for wireless data transfer.

UWB Ultra-Low-Power Short Distance FIR Spectrally Shaped NRZ Transmission 


\section{Design Considerations Of A Wireless UWB Transceiver}

\subsection{Chapter Overview}

This chapter provides an in-depth study of design procedures and implementation challenges of ultra wideband wireless transceivers.

\subsection{Pulse Generation}

In single band or impulse radio (IR) based ultra wideband transmission very small pulses of the order of nanoseconds are used for information transfer.Generating such short duration pulses can be a challenge. There are various techniques that are currently used for sub nanosecond pulse generation. One method to generate UWB pulses is fast activation and deactivation of semiconductor switches. However, because of the sophisticated and costly arrangement required for pulse generation by the above method, this technique is seldom used for UWB communication systems. 
Another more reliable method to generate UWB pulses is by using tunnel diodes, step recovery diodes and drift step recovery diodes. The pulses generated by using any of these methods are usually step like or ramp like pulses and do not fit into the spectral mask prescribed by FCC for UWB communication. Hence, proper pulse shaping circuits are employed to rectify the generated pulses to acquire the spectral mask as prescribed by FCC.

The most commonly used pulses for UWB communication are Gaussian pulse and its derivatives. Many circuits are reported in literature for production and appropriate shaping of such pulses. The literature review section of the same chapter provides a more detailed discussion on the circuit architectures reported so far.

\subsection{Modulation Options}

Various modulation options exists for UWB communication. Selecting an appropriate modulation scheme depends largely on the application, design specifications, constraints and range as well as hardware complexity, transmission and reception power, data rate, channel capacity and reliability. The most commonly used modulation schemes for UWB communication are On-Off Keying (OOK), Pulse Amplitude Modulation (PAM), PulsePosition Modulation (PPM), Binary Phase Shift Keying (BPSK), Transmitted- Reference Modulation (TR) \& M-ary PAM. 


\subsubsection{Pulse Amplitude Modulation}

Pulse Amplitude modulation involves changing the amplitude of the pulses based on the data bits to different power levels. In this modulation scheme, a higher power pulse encodes to data bit 1 and a lower power pulse encodes to a data bit 0 . The equation defining PAM is as below [6]:

$$
y(t)=\sum_{K=1}^{N} A_{s K^{x(t-K T)}}
$$

where,

$$
\begin{aligned}
& K=\text { Number of transmitted bit } \\
& T=\text { Pulse repetition period } \\
& s_{K}=\text { Nth bit (high or low) } \\
& x(t)=\text { Ultra wideband pulse }
\end{aligned}
$$

and

$$
A=\text { The voltage level associated with each bit }
$$

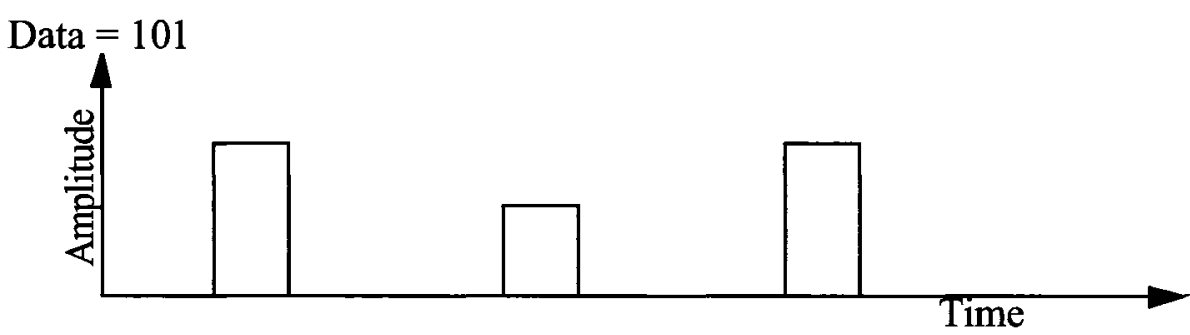

FIGURE 3.1 Pulse Amplitude Modulation 


\subsubsection{On-Off Keying}

On-Off keying is one of the simplest forms of modulation. It is a special case of PAM where the presence of a pulse encodes to data bit 1 and the absence of a pulse encode to data bit 0 . The equation [6] and figure are as follows:

$$
y(t)=\sum_{K=1}^{N} s_{K} \cdot x(t-T)
$$

where,

$$
\begin{aligned}
& K=\text { Number of transmitted bit } \\
& T=\text { Pulse repetition period } \\
& s_{K}=\text { Transmitted bit level (high or low) } \\
& x(t)=\text { Ultra wideband pulse }
\end{aligned}
$$

and

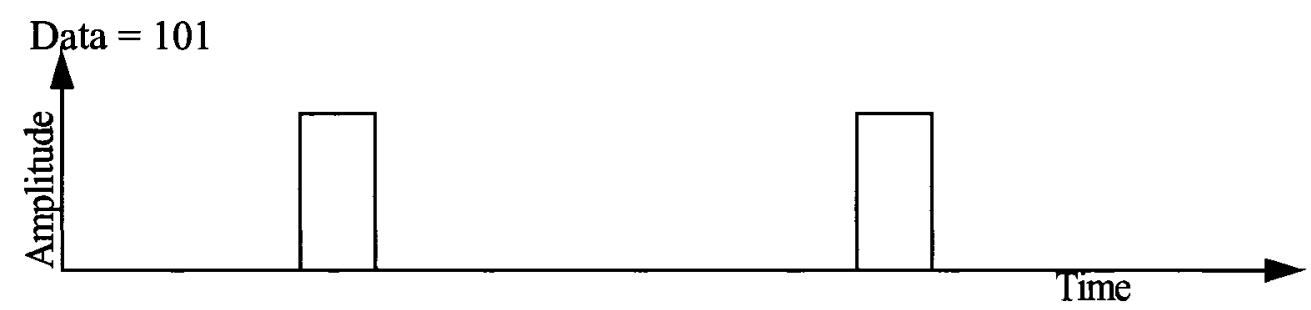

FIGURE 3.2 On - Off Keying

\subsubsection{Binary Phase-Shift Keying (BPSK)}

Binary Phase shift Keying or BPSK modulation is the most popular modulation scheme 
for UWB. It is also a special case of PAM where the change of polarity in the transmitted pulses encodes to data bits 1 or 0 . For instance, a pulse with positive polarity corresponds to data bit 1 whereas a pulse with negative polarity corresponds to data bit 0 . The equation and figure are as follows [6]:

$$
y(t)=\sum_{K=1}^{N} s_{K} \cdot x(t-K T)
$$

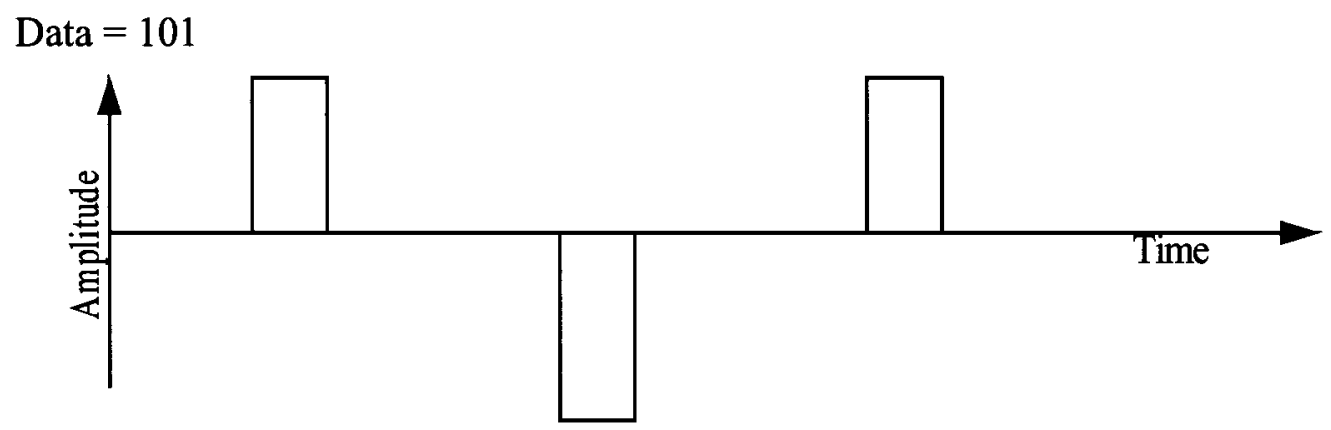

FIGURE 3.3 Binary Phase Shift Keying

\subsubsection{Pulse Position Modulation (PPM)}

The pulse position modulation scheme involves the change in the position of pulse depending on the transmitted data while the amplitude and pulse phase remains the same. The equation [6] and figure are as follows: 


$$
y(t)=\sum_{K=1}^{N} x\left(t-K T-\left(s_{K} \cdot \delta\right)\right)
$$

where,

$$
K=\text { Number of transmitted bit }
$$

$T=$ Pulse repetition period

$s_{K}=$ Transmitted bit level (high or low)

$x(t)=$ Ultra wideband pulse

and

$\delta=$ Modulation index that provides the time shift

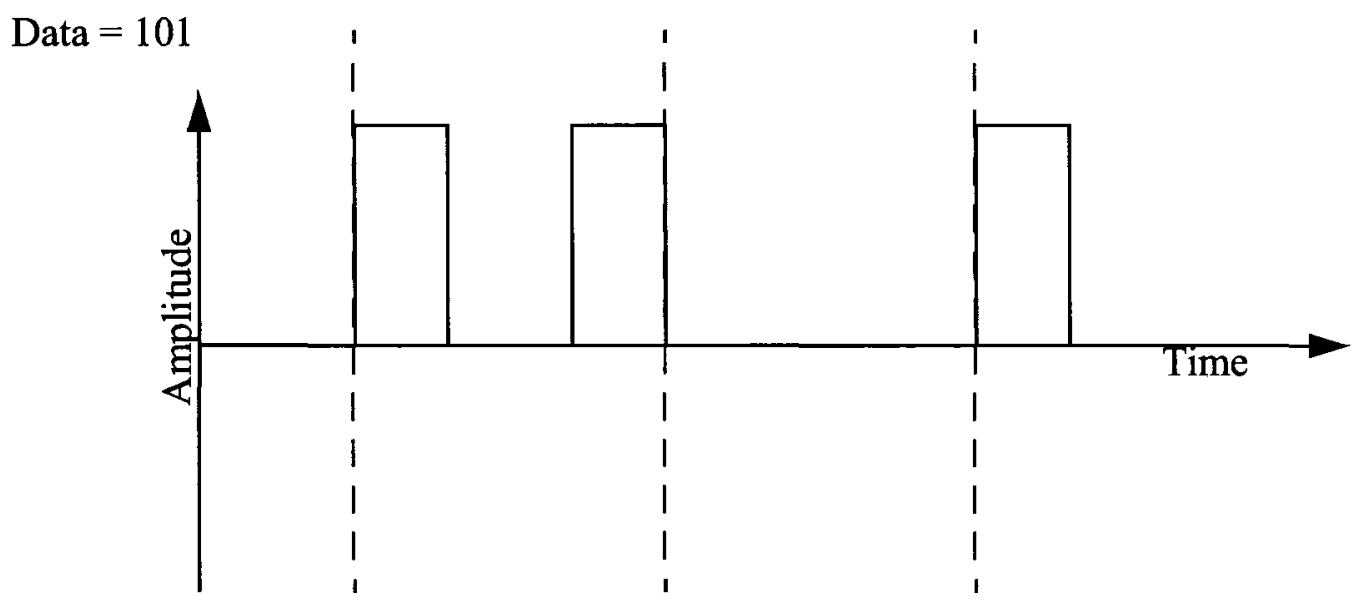

FIGURE 3.4 Pulse Position Modulation 


\subsubsection{Transmitted Reference Modulation}

Transmitted- Reference Modulation scheme is quite new to UWB technology, however due to its simplicity and robust performance in multipath channels it is becoming a popular modulation scheme for UWB. In TR modulation, information is transferred in the form of doublets. The first pulse of the doublet is a reference pulse and does not contain any information. The second pulse which is sent after some delay $\mathrm{D}$ is the modulated pulse and it carries the data information. For instance, a reference and data pulse of the same polarity can be encoded to a binary 0 and that of opposite polarity as binary 1 or vice versa. The equation [6] and figure are as follows:

$$
y(t)=\sum_{K=1}^{N}\left[x(t-T(K-1))+\left(2 s_{K}-1\right) x(t-D-T(K-1))\right]
$$

where,

$$
\begin{aligned}
& \text { where, } \\
& \qquad \begin{array}{l}
\text { K }=\text { Number of transmitted bit } \\
T=\text { Pulse repetition period } \\
s_{K}=\text { Transmitted bit level (high or low) } \\
x(t)=\text { Ultra wideband pulse } \\
D=\text { Delay between the reference and data pulse }
\end{array} \\
& \text { and } \quad
\end{aligned}
$$


Data $=101$

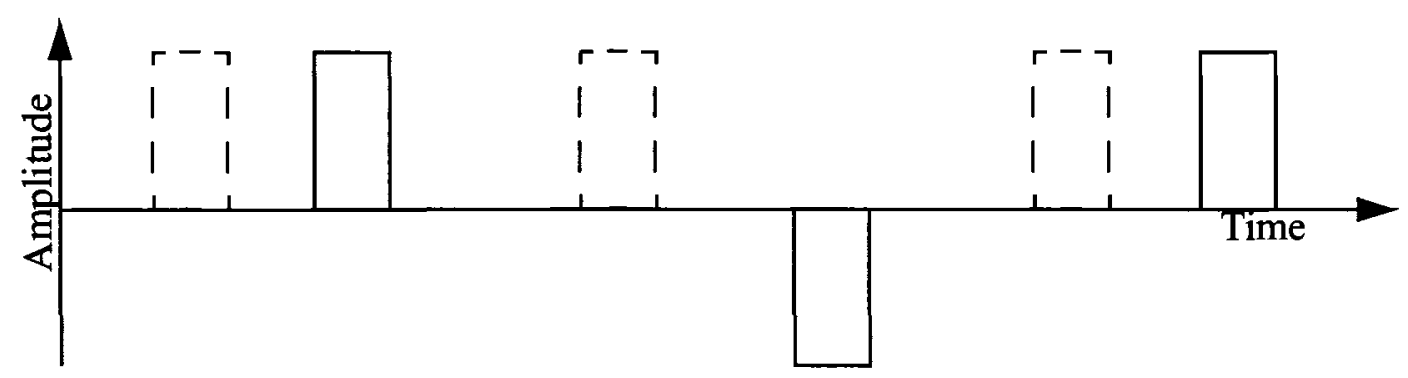

FIGURE 3.5 Transmitted Reference Modulation

\subsubsection{M-ary Modulation}

Higher data rates can be achieved by mapping multiple bits on UWB pulses which leads to M-ary Modulation schemes. M-ary PAM, M-ary PPM and biorthogonal signaling are some of the most common M-ary Modulation methods. The figure below shows the modulation schemes.

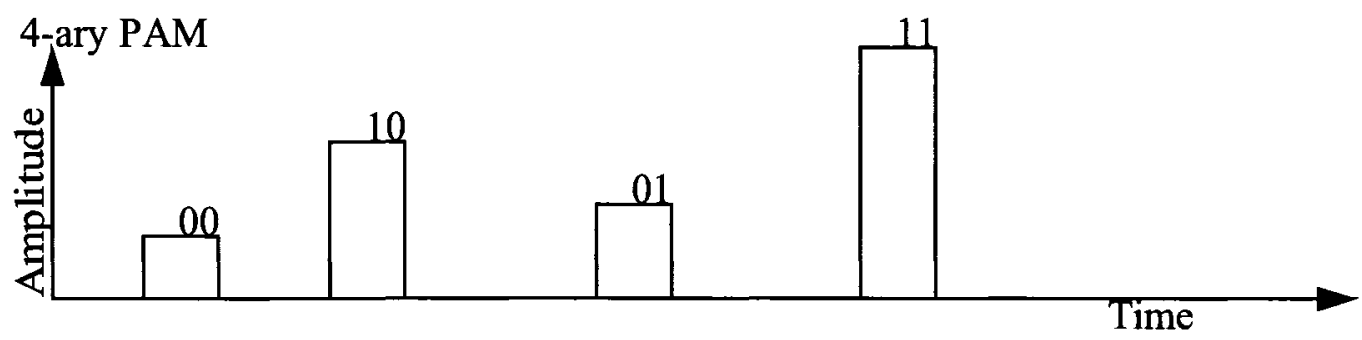

FIGURE 3.6 M-ary Modulation

UWB Ultra-Low-Power Short Distance FIR Spectrally Shaped NRZ Transmission 


\subsection{Antenna Issues}

Antennas are transducers that are designed to transmit and receive electromagnetic radio waves. Selection and design issue of UWB antennas is largely reliant on three main factors: UWB system-whether its single band or multiband, chosen modulation scheme and the targeted application.

In general, for multiband systems it is desirable that along wide impedance bandwidth in the targeted band, the antenna also provides constant gain and constant polarization in the desired direction. Whereas for single band system, linear phase response of the antenna is also critical which provides equal group delay and hence minimum pulse shape distortion.

Another critical factor is the design and implementation specifications. UWB antenna can be broadly classified as omni-directional, directional, miniature (to be used on PCB), array based or can be specifically designed for targeted application such as high and low communication systems, medical imaging systems or vehicular radar systems. Omni directional antennas have low gain, wide field of view and small size whereas directional antennas have high gain, are larger in size and have a narrow field of view. The antenna chosen should compensates for the path loss and must be specifically tailored in both impedance matching as well as spectral response to contribute to overall system performance. Nowadays, much emphasis is being given on small size antennas that can be used for wireless chip-to-chip communication at the same time supporting high data rate transmission.

UWB Ultra-Low-Power Short Distance FIR Spectrally Shaped NRZ Transmission 


\subsection{Impulse Radio Transmitter Design Issues}

A typical impulse radio or IR transmitter is shown in figure 3.7. The main issues that are associated with an impulse radio transmitter are pulse generation and pulse shaping. Mostly band pass filters are used to properly shape the generated pulse. A detailed discussion about various suggested IR based transmitters can be found in the literature review of the same chapter.

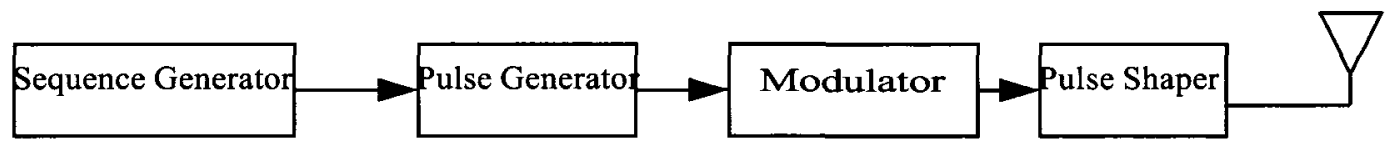

\section{FIGURE 3.7 Typical IR Transmitter}

\subsection{OFDM Transmitter Issues}

OFDM transmission employs the channelized approach for transfer of information on UWB bandwidth. The designing of an efficient OFDM transmitter is quite a challenging task. The main reason being relatively complex and power consuming building blocks then those that are used in IR transmitter. Typical blocks include convolution encoder, bit 
interleaver, IFFT calculating block, DAC and a power amplifier. Figure 3.8 shows the block diagram of an OFDM transmitter [12].

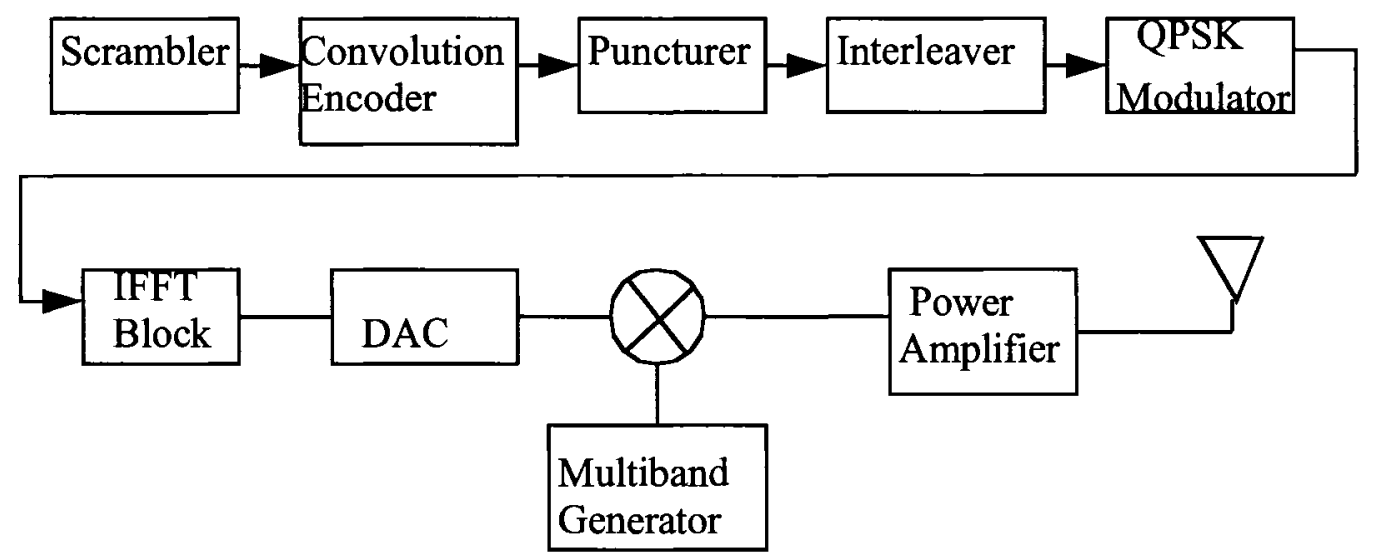

FIGURE 3.8 Typical OFDM Transmitter

\subsection{Receiver Architecture}

Transceivers for UWB communication must be simple, power-efficient, economical, small in size as well as capable of high rate data transmission. To design a transceiver with all these properties is indeed quite a challenging task. Several options for UWB receivers are proposed in the literature. In general, they can be categorized as coherent and noncoherent receivers. 
A coherent receiver for UWB usually employing matched filtering and rake reception has a complicated circuitry and involves a large number of computation that can result in

high power dissipation. For optimal operation, coherent receivers desire several parameters along with the received signal such as channel and interference characteristics, multipath delays, channel coefficients for each path and pulse shape estimation after distortion.

In comparison, non-coherent receivers needs relatively trivial amount of information to fully detect and regenerate the transmitted information. Specific parameters such as synchronization, channel estimation and pulse shape estimation that were compulsory in the case of coherent receivers are not required for information detection in the case of noncoherent receivers. The non-coherent receivers include TR-based receivers, energy detectors and differential detector. All of them work on the basic principle of receiving some reference pulse and the information pulse is sent and detected on the basis of the distance or position from the reference pulse. It can be easily concluded that such receivers are also immune to timing mismatch.

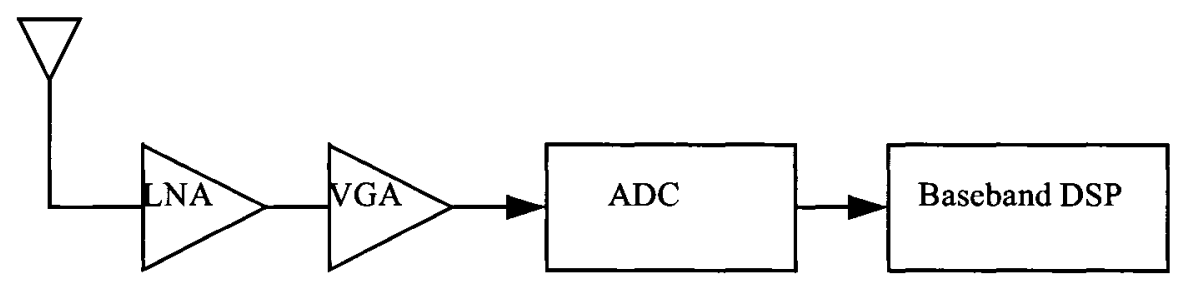

FIGURE 3.9 Typical IR Receiver

UWB Ultra-Low-Power Short Distance FIR Spectrally Shaped NRZ Transmission 


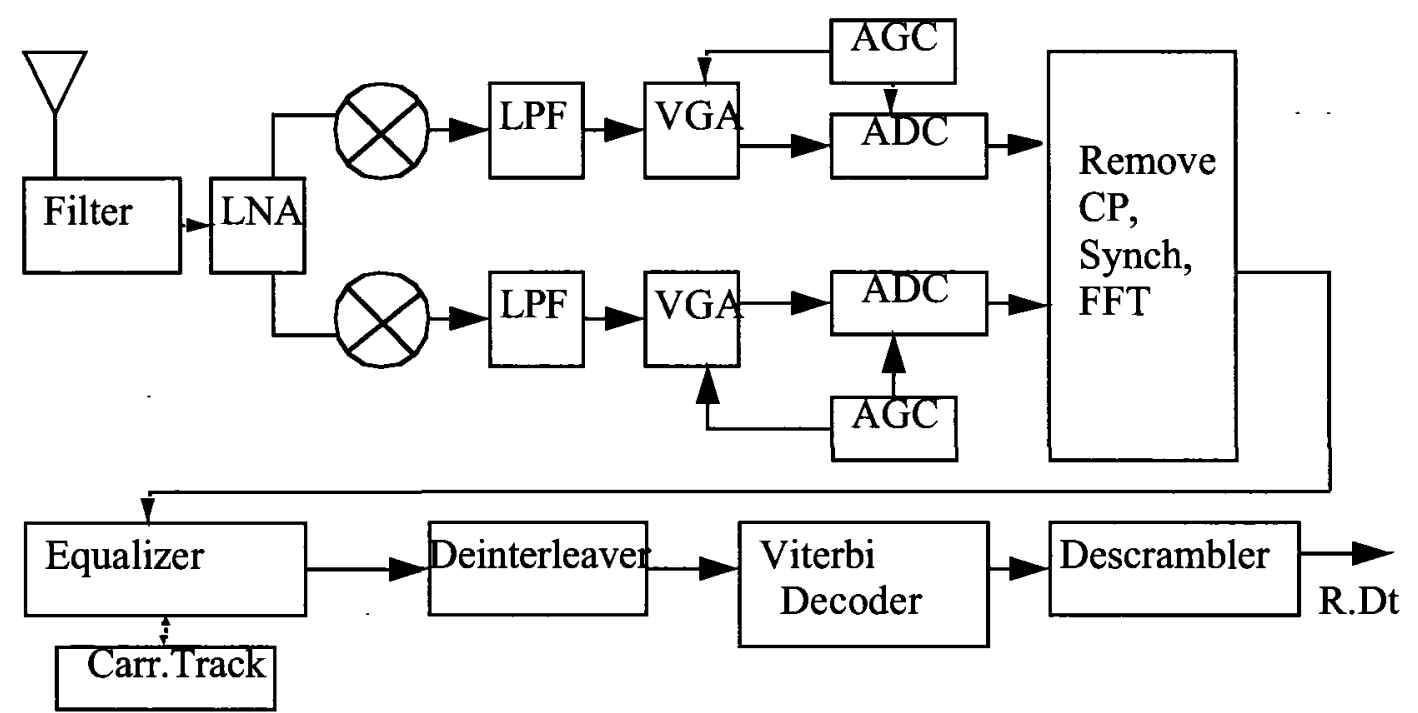

FIGURE 3.10 Typical OFDM Receiver

\subsubsection{Sampling}

Sampling is a critical issue for receiver implementation in UWB. Since absolute detection of the transmitted signal requires a sampling rate of at least twice the Nyquist rate at the receiver end. In addition to such high sampling frequency, the ADC conversion must also support a very large range of frequencies to entirely determine the signal. This also means that a significantly high speed signal processing is required which may result in the usage of costly, high power DSP processors.

\subsubsection{Synchronization}

Synchronization between the transmitted and received pulses is another important issue in receiver design. Synchronization operation can be defined as a search operation where the 
receiver searches for the correct timing of the transmitted signal and locks onto it, without an accurate timing synchronization demodulation and data detection are not possible. Synchronization is more significant in coherent receivers where the channel delay and correct pulse timing are important for fast and accurate acquisition. On the other hand, in noncoherent receivers a slight error in the timing estimation does not degrade the system performance and allows the use of less complex synchronization algorithms.

\subsubsection{Multipath Coefficient Estimation}

The estimation of multipath characteristics such as the delay of each path and the coefficients of each path is a difficult task. Even for coherent receivers, the coefficients for selected taps are estimated. The channel estimation can be avoided for non-coherent receivers.

\subsubsection{Pulse Shape Estimation}

Pulse shape estimation is an important phenomenon for coherent receivers that employs matched filters. In matched filters, the received bits are correlated with the locally generated pulse template to decode the information. For generating the local template, the knowledge of the pulse shape after passing through the channel is important. The is achieved usually by sending a single pulse or a train of pulses through the channel and studying the changes that has been introduced in it while passing through the channel. The non-coherent receivers do not need pulse shape estimation. 


\subsubsection{Interference in Ultra Wideband}

UWB receiver performance can be severely degraded by interference. These interferences can be characterized as Inter symbol interference (ISI), Inter frame interference (IFI), Multiple access Interference (MAI) and Narrowband interference (NBI). Correlation receiver can compensate for such interferences by adding the characteristics in the generated templates, however this means that it will also require prior information about the interference statistics.

\subsubsection{Modulation Options and Application Specific Issues}

Apart from the cost and complexity issue the choice of receiver also depends upon the modulation scheme chosen at the transmitter end. Some modulation schemes such as OOK, PAM, PPM and M-ary PPM enables non coherent demodulation whereas schemes such as BPSK, M-ary PAM and QAM require coherent demodulation. Application specific issues such as data rate, required channel capacity, power dissipation and cost are also important for selection of receivers.

\subsection{Channel Modeling}

Channel Modeling is vital for any wireless communication network. In any wireless system, the signal arriving at the receiver is composed of both line of sight (LOS) and non line of sight (NLOS) components. The NLOS components are formed due to scattering, UWB Ultra-Low-Power Short Distance FIR Spectrally Shaped NRZ Transmission 
diffraction and reflection of the transmitted signal. In UWB communication, the factor of large bandwidth makes channel modeling much more significant. Traditional narrowband channel modeling estimates the signal distortion only due to path loss and hence are not suitable for UWB channels where complete information of all the channel parameters such as path loss, multipath components and delay estimation are needed to utterly model a channel. Although UWB channel modeling is an evolving area of research nowadays, the UWB channel models can be widely divided as deterministic and statistical. Using simulation tools that absolutely defines the propagation properties of a signal crossing a specific geometry creates deterministic models. The major advantage of these simulationbased models is that once the physical properties of a channel are known, it can yield accurate results, whereas the disadvantage is that any change in environment's physical parameters means re-calculating the channel model and characteristics which makes it computational complex procedure. Generating the artificial estimation of the channel responses by setting appropriate channel parameter values forms the statistical modeling of channel. Usually, statistical models are simpler than deterministic models. For an optimized performance of the system, UWB propagation channel estimation is much desirable.

\subsection{Literature Review}

This section discusses the relative issues and design specification for UWB transceivers that are reported in literature. 
The review starts with a study of various pulse generator circuits that are used for IR trans matters. Pulse generation circuits are employed to generate sub-nano second pulses with very low duty cycle. Such circuits are used in extremely low power applications. Most circuits reported in literature for pulse generation are all digital circuits. Reference [9] proposes transmitter module containing an RF clock generator, an UWB pulse generator and an UWB antenna. The RF clock generator is composed of quartz oscillator, a delay locked loop (DLL) and a digital edge combiner for generating the clock signal that drives the pulse generator. The pulse generator is a digital circuit consisting of delay line, two NAND gates and two cross coupled differential pair with $50 \mathrm{Ohms}$ load. The digital circuit is driven by $533 \mathrm{MHz}$ clock signal which passes through four different delay paths. One with one inverter delay, one with four inverter delays, one with three inverter delays and one with six inverters delay. Positive pulses are obtained when one $\&$ four inverter delays are NAND-ed together and negative pulses are obtained when the three and six inverter delays are NAND-ed. The differential pair is used to adjust the width of the pulse. The pulses obtained are mono-Gaussian pulses and a band-pass filter is employed to shape the power spectrum according to FCC mask. The receiver is based on a non-coherent, energy-based approach which is simpler to implement since it doesn't use any correlator bank. It is composed of LNA, VGA Gilbert-based multiplier, integrator, one FLASH ADC and DSP block. The total power consumption of the transmitter is reported to be $20.48 \mathrm{~mW}$ with $3.3 \mathrm{~V}$ power supply. The power consumed by the receiver is $130 \mathrm{~mW}$. The limitation of the above reported architecture is the complex transmitter circuitry and not 
using coherent receiver results in noise also being amplified at the receiver. The circuit was implemented using $0.35 \mu \mathrm{m}$ process.

Reference [10] proposes another pulse generator which generates the 5th derivative of Gaussian pulse. Since the power spectrum of such pulses complies completely with the FCC regulations. The authors propose to use such pulses directly for transmission without the use of any filters. The circuit consists of a digital triangular pulse generator and an output stage. The triangular generator identify the voltage variations at each node to generate the positive and negative triangular pulses which are added at the output stage to realize the desired pulse. The circuit is designed using $0.18 \mu \mathrm{m}$ CMOS technology and the reported power consumption is $15.4 \mathrm{~mW}$ and $675 \mu \mathrm{W}$ at the pulse repetition frequency of 500 and $1 \mathrm{MHz}$ respectively with $1.8 \mathrm{~V}$ power supply. Reference [11] proposes another impulse generator for IR-UWB system. The impulse generator consists of four delay blocks and an XOR gate for pulse generation and a FIR filter for the impulse shaping so that the resultant pulse satisfies the spectral emission mask requirement specified by FCC. However, the entire work is simulated using MATLAB and ADS and no details about the power consumption or the filter components were reported.

Reference [12] studies the design considerations for low-power UWB receivers. The author first compares the three approaches for IR -based architecture. The time interleaved approach, Frequency Domain Channelization and Analog Domain signal processing. High data transmission rate requires high sampling rate $\mathrm{ADC}$ at the receiver end. Time-interleaved architecture usually employ an interleaved configuration of ADCs to relax the sam- 
pling frequency requirement. The analog-to-digital conversion happens right after the LNA and VGA and the complete signal processing from signal detection to correlation is realized in the digital domain. The second approach is to achieve channelization using a bank of mixers operating at equally spaced frequencies to decompose the analog input signal into subbands. This greatly relaxes the $\mathrm{ADC}$ requirements since each $\mathrm{ADC}$ sees only the bandwidth of the subband signal which is a fraction of the whole signal bandwidth. The third method is to move the signal processing in the analog domain where mixers and integrators are employed to correlate the received signal with the generated template. Finally, a generic multi band receiver was also introduced. The reported comparison showed that the power consumption is lowest in the analog configuration whereas, frequency channelization approach consumes highest power.

A mostly-digital ultra wideband transceiver was proposed in [13]. In this architecture, time interleaved configuration employing one bit ADC's was adopted. The backend was all digital and utilizes matched filters. Authors have suggested that use of one bit ADC can significantly reduce the total power consumed by the transceiver hence making it more suitable for sensor network applications.

In [14] and [15]channelized ultra wideband receivers are proposed. The received signal is divided into sub bands by mixers and filters. The digital filters perform matched filtering, noise whitening and equalization to estimate the spread code directly based on the sampled signal. Time domain reconstruction is done by performing inverse fast fourier trans- 
form (IFFT). This approach is the most complex approach resulting in highest power dissipation.

The reported architecture in [3] uses analog domain approach for signal processing. The power consumption of transmitter is reported to be only $1 \mathrm{~mW}$ while the receiver consumes almost $23 \mathrm{~mW}$. The transmitter has only pulse generator. The receiver consists of wideband LNA, multiplier, analog template signal generator, integrator and ADC. The power management block has a wake-up circuit and works in either sleeping mode or working mode. The timing circuit controls the pulse generation and system synchronization (acquisition \& tracking). 1-bit ADC is used which is actually a regenerative latch. No description of baseband block was given. Reference [16] proposes a direct-conversion UWB transceiver for OFDM applications employing three resonant circuits and three phase locked loops. Because of a common gate input stage, the receiver allows direct sharing of the antenna with the transmitter. The reported power dissipation is $105 \mathrm{~mW}$ from a $1.5 \mathrm{~V}$ supply. A time interleaved approach for Analog to digital conversion is adopted in [17]. It employs 4-bit resolution FLASH ADC's sampling at $1.2 \mathrm{Gsa} / \mathrm{s}$. Each ADC works at $300 \mathrm{MHz}$. The digital backend adds group of four consecutive samples and treats them as a single sample. Since the backend has employed 10 parallel correlators, the backend works at a reduced data rate of $30 \mathrm{MHz}$. The total power consumption is $275 \mathrm{~mW}$.

An ultra wideband TAG circuit Transceiver was reported in [18]. The receiver is a noncoherent receiver so it is highly independent of the shape of pulse. A 4-bit ADC is used. The circuit is used for the reported data rate of $1 \mathrm{kbps}-100 \mathrm{kbps}$. Integrators are used for UWB Ultra-Low-Power Short Distance FIR Spectrally Shaped NRZ Transmission 
energy collection and the control circuitry for accurate turn-on and turn-off operation of the integrator is all digital based on eight three-bit counters. After the integrator selection is done by automatic gain control, two energy collection values out of integrator are feed into detection / bit decision logic block of comparators. The baseband DSP logic implements the MASTER / SLAVE functionality of the UWB TAG. The transceiver has not yet been fabricated and no details about the power consumption were provided. A CMOS UWB IR Transceiver for $1 \mathrm{Mb} / \mathrm{s}$ Data Communication and $+/-2.5 \mathrm{~cm}$ range finding is reported in [19]. The power consumption of the transmitter is $0.7 \mathrm{~mW} @ 2.5$ Volts and $0.3 \mathrm{~mW}$ at 1.8 volts, whereas the total power consumed by receiver without LNA bias switching is $4.0 \mathrm{~mW}$ and with LNA bias switching is $0.3 \mathrm{~mW}$.The transmitter is all digital. The building blocks of transmitter are pulse generator and antenna. The author proposes that the use of a CMOS output buffer drives the antenna directly hence eliminating the need of an analog power amplifier. OOK is the chosen modulation scheme. The receiver is composed of LNA and clocked correlator. The clocked correlator saves the area and power required for an over $1 \mathrm{GHz} \mathrm{ADC}$. The synchronization and data acquisition is done by delay controller block embedded inside clocked correlator. Reference [20] proposes another non-coherent analog domain signal processing transceiver structure. The transmitter consumes $41.4 \mathrm{~mW}$ average power whereas the receiver consumes $29 \mathrm{~mW}$ average power. The entire signal processing for the receiver is done in analog domain. The receiver does not have analog correlators, instead it is composed of LNA, multiplier, a demodulation driving amplifier (DDA) and a LPF. PAM is used for modulation.

UWB Ultra-Low-Power Short Distance FIR Spectrally Shaped NRZ Transmission 
In [21], the author discusses two different algorithms for arrival time estimation of data.

The algorithms are threshold crossing and the maximum selection algorithm. The proposed structure is non-coherent structure. The processing is accomplished in analog domain. The drawback in energy-collection receiver is that interference and noise also gets amplified. Synchronization is achieved in two steps, first coarse synchronization is done to detect the range and then fine synchronization is done to detect the peak. Both uses same set of integrators. The reported power consumed in the receiver is $48 \mathrm{~mW}$.

Another Multi-band UWB transceiver design was presented in [22]. The adopted modulation scheme is spectral-keying modulation. For the MB-UWB system, a block-wise multipath channel model was developed using FIR filter block. At the transmitter end, a modulated symbol is sent through sub symbol pulses that have distinct frequencies. At the receiver, a minimum mean square error (MMSE) receiver bank followed by a simple mapping-based decision block is used to detect the received signals. The details about the receiver architecture were given. The discussion dwells largely upon modulation and demodulation of the signal. Reference [23] reports a low complexity diversity receiver for either single or multiband operation. The diversity receiver is capable of collecting multipath diversity by sampling at fraction of Nyquist rate. The receiver is operational with single band or multiband UWB transmitter. The paper has adopted the asymmetric single band UWB transceiver designs with different ADC / DAC rates. In addition, a low sampling rate single band receiver was deployed in conjunction with the Multi band transmitter. Correlation operation was carried out in analog domain. 
A sub-sampling UWB radio has also been reported [24] where the receiver front end down converts the signal frequency by sub-sampling, thus requiring less hardware. Analytical signaling is used. The digital backend consists of analytic signal transform, correlators, analytic matched filters and pulse estimator. The signal is detected through matched filters and the correlation block is used to provide additional gain or despread any coding.

TABLE 3. 1 Comparison With other Reported Work

\begin{tabular}{|c|c|c|c|c|c|}
\hline References & Methodology & Data Rate & Power $(m W)$ & Energy/bit & Area $\left(\mathrm{mm}^{2}\right)$ \\
\hline [9] & $I R$ & $4 \mathrm{Mb} / \mathrm{s}$ & $20.48 @ 3.3 V$ & $5.12 n J$ & 0.16 \\
\hline$[10]$ & $I R$ & $\begin{array}{l}500 \mathrm{MHz}(P R F) \\
1 \mathrm{MHz}(P R F)\end{array}$ & $\begin{array}{l}15.4 @ 1.8 \mathrm{~V} \\
0.675 @ 1.8 \mathrm{~V}\end{array}$ & - & - \\
\hline [16] & $O F D M$ & $480 \mathrm{Mb} / \mathrm{s}$ & 105@1.5V & $218 p J$ & 1 \\
\hline [19] & $I R$ & $1 \mathrm{Mb} / \mathrm{s}$ & $1 @ 1.8 \mathrm{~V}$ & $\ln J$ & 0.04 \\
\hline$[20]$ & $\begin{array}{l}\text { Analog } \\
\text { signal } \\
\text { processing }\end{array}$ & $50 \mathrm{Mb} / \mathrm{s}$ & $41.4 @ 1.8 \mathrm{~V}$ & $828 p J$ & $\begin{array}{l}1.085 \mathrm{x} \\
0.79\end{array}$ \\
\hline$[31]$ & $802.15 .4 a$ & $\begin{array}{l}1 \mathrm{Mb} / \mathrm{s} @ 3.1 \mathrm{G} \\
1 \mathrm{Mb} / \mathrm{s} @ 10 \mathrm{G}\end{array}$ & $\begin{array}{l}0.65 @ 1 V \\
1.4 @ 1 V\end{array}$ & $\begin{array}{l}0.65 n J \\
1.4 n J\end{array}$ & - \\
\hline [32] & $I R$ & $1.16 \mathrm{~Gb} / \mathrm{s}$ & $12.6 @ 1.8 \mathrm{~V}$ & $10.86 \mathrm{pJ}$ & 0.1387 \\
\hline [33] & $I R$ & $1 \mathrm{~Gb} / \mathrm{s}$ & $108 @ 1.8 \mathrm{~V}$ & $108 \mathrm{pJ}$ & - \\
\hline$[34]$ & $I R$ & $160 \mathrm{Mb} / \mathrm{s}$ & $10 @ 1.2 \mathrm{~V}$ & $62.5 \mathrm{pJ}$ & 1.56 \\
\hline [35] & $I R$ & $100 \mathrm{Mb} / \mathrm{s}$ & $180 @ 3.3$ & $1.8 \mathrm{~nJ}$ & $1.5 \times 1.3$ \\
\hline$[36]$ & $\begin{array}{l}\text { Phased } \\
\text { Array }\end{array}$ & $480 \mathrm{Mb} / \mathrm{s}$ & $\begin{array}{l}\text { Tx sing. } 397 \\
\text { Tx dual. } 435\end{array}$ & $\begin{array}{l}0.827 \mathrm{~nJ} \\
0.906 \mathrm{~nJ}\end{array}$ & $4.2 \times 3.8$ \\
\hline$[37]$ & $I R$ & $\begin{array}{l}10 \mathrm{Mb} / \mathrm{s} @ 10 \mathrm{~m} \\
250 \mathrm{~kb} / \mathrm{s} @ 30 \mathrm{~m}\end{array}$ & 29.7 & $\begin{array}{l}2.97 n J \\
118 n J\end{array}$ & 0.4 \\
\hline$[38]$ & $I R$ & 1.8 Gpulses $/ \mathrm{s}$ & $227 @ 1$ & $126 \mathrm{pJ} / \mathrm{pulse}$ & 2.83 \\
\hline This work & Serial Data & $10 \mathrm{~Gb} / \mathrm{s}$ & $53.6 @ 1.2 \mathrm{~V}$ & $5.36 \mathrm{pJ}$ & $0.1 \times 0.13$ \\
\hline
\end{tabular}


The table above provides a comparison of this work with other reported work. It can be seen that the work carried out in the thesis is most efficient in terms of energy per bit and data rate.

\subsection{Proposed Approach For Serial Data Transmission in UWB}

\section{Spectrum}

As gathered from the literature review, almost all of the pulse based transmission of UWB signals employs some sort of pulse shaping circuitry to tailor the pulse shape in order for it to justify the UWB spectral mask. Reference [9] and [11] have both used a FIR filter to shape the final pulse so that the resultant frequency spectrum is in accordance with the emission limits. It should however be noted that some of the reported work like [10] utilizes the direct transmission of pulses over UWB channel. The generated pulses in this case already fits in the required spectrum mask.

The proposed work in this thesis dwells upon the data transmission making use of a band pass FIR filter to accomplish the bandwidth specifications. The proposed structure serves as a bridge between serial data transmission and ultra wideband communication. The block diagram of the proposed interface is shown in figure 3.11. The transmitter end has a sequence generator and a FIR filter block consisting of a shift register, XOR gates and transmitter driver. The wireless communication is achieved through sacked inductors 
which are electromagnetically coupled together and the receiver end is established by using a CDR circuit for data recovery.

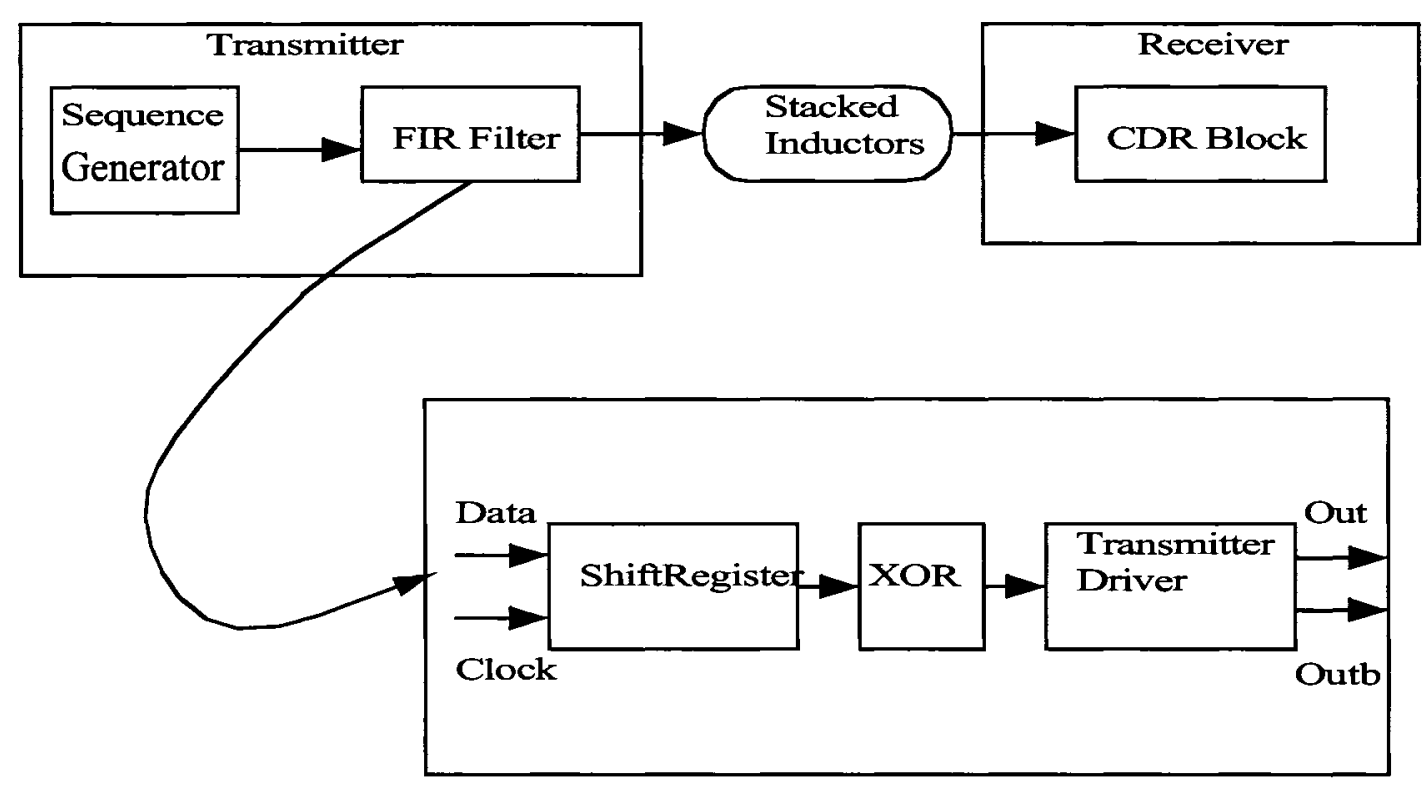

FIGURE 3.11 Serial Data Transceiver Interface For UWB Communication

\subsection{Design Aspects For FIR filter}

The typical structure chosen for implementing FIR bandpass filter consists of widely used communication blocks and is pretty similar to what has been reported in the literature repeatedly. To establish FIR filtering one has to accomplish three basic operations data shifting or creating a delay, multiplication and summation. The structure to achieve this purpose is derived from [26], [27] \& [28]. The driver structure is similar however, the 
specifications such as filter rate, number of tap and clocking techniques are modified according to the circuit requirements.

\subsubsection{Sampling Frequency}

According to the sampling theorem [29], in order to reconstruct a signal without distortion it should be sampled at a rate which is twice the maximum frequency of the signal. The filter bandwidth for ultra wideband signal is almost $7.5 \mathrm{GHz}$ with high and low cut-off frequencies of 3.1 and $10.6 \mathrm{GHz}$. The required sampling frequency is therefore, $20 \mathrm{GHz}$ implementing which is quite a challenge in itself. The clock is externally provided for generating high sampling frequency.

\subsubsection{Order of Filter}

A high order filter is essential for sharp transition at high and low cut-off frequencies so that the spectrum is closer to the desired spectral mask. To accomplish this task for such wide bandwidth, a high order filter consisting of 20 taps was designed. The resulting filter response is quite in accordance with the desired mask. The increased number of taps however, have boasted the required lay out area but choice of optimum quantized coefficients has kept the power dissipation a minimal of $8 \mathrm{~mW}$ for the entire structure.

UWB Ultra-Low-Power Short Distance FIR Spectrally Shaped NRZ Transmission 


\subsubsection{Quantized Optimum Filter Coefficients}

MATLAB filter design and analysis tool call FDA tool was used to generate the optimum coefficients so that the resultant filter displays optimum response. The generated coefficients were further quantized and normalized for transistor level implementation. Another filtering structure reported in [27] is used for serial data on-board and board-to-board transmission with the difference that the coefficients were adaptive in the reported case, whereas in order to follow the spectral mask, the coefficients of the designed filter are fixed.

\subsubsection{Flexibility}

The employment of XOR gates in the filter structure has introduced some implementation flexibility. The XOR gates are used for polarity control of the data passing through the filter. Polarity control has enabled the same filter to be used for a different application or can be used to establish the same filter with a different set of coefficients in case a need to alter them arises.

\subsection{Design Aspects Of Stacked Inductors}

Stacked inductors are designed and extracted by using the software called ASITIC. The inductors can be used for chip-to chip or same board wireless communication. The chosen methodology was to design and use stacked inductors that are embedded in different metal UWB Ultra-Low-Power Short Distance FIR Spectrally Shaped NRZ Transmission 
layers of the same technology. The specifications and design considerations are discussed below:

\subsubsection{Layout Area}

The most significant feature for the inductors is the layout area it covers. The goal is to accomplish the wireless communication with minimal inductive area. Typically, the metal thickness and the minimum spacing are used to determine the quality factor of the inductor whereas the hollow area enhances the quality factor. For achieving a balance between all these aspects, inductors with the metal thickness of $12 \mu \mathrm{m}$ are used with inner spacing only of $1 \mu \mathrm{m}$ and a hollow area of $50 \times 50 \mu \mathrm{m}^{2}$. The inductor has 5 turns and the total layout area is found to be $218 \times 218 \mu \mathrm{m}^{2}$.

\subsubsection{Inductive Coupling}

Excellent inductive coupling is very significant for transmission and reception of data with least number of corrupted bits. The alignment of inductors is another important factor to get optimum coupling. Generally, a coupling coefficient closer to 1 indicates excellent mutual coupling. 


\subsubsection{Quality Factor}

Quality factor of a system is defined as the ratio between the frequency at which the system is oscillating and the rate of energy dissipation by the system. A high quality factor indicates that the system is dissipating energy slowly and is desirable.

\subsubsection{Alignment}

According to [30], alignment is important for wireless chip-to-chip transmission. In an aligned system, each transmitting plate lines up perfectly with a receiving plate, maximizing the signal coupled between the two. With misaligned chips, a transmitter pad will couple into other receiver pads, simultaneously reducing the intended signal and increasing cross talk to the other receiver pads. 


\section{Implementation \& Evaluation of UWB \\ Channel}

\subsection{Chapter Overview}

This chapter introduces the fundamental building blocks and the test bench set up. In ASIC design, transistor level designing and simulation results are expressed as implemented whereas manufactured circuit and measured results are termed as fabricated. Therefore, these terms will be used hereafter for circuit description as appropriate. Performance analysis of the complete circuit structure concludes the chapter.

\subsection{Implementation Challenges}

High speed filtered wireless serial data transmission targeting the ultra wideband signal bandwidth of $7 \mathrm{GHz}$ from $3.1 \mathrm{GHz}$ to $10 \mathrm{GHz}$ in accordance with the FCC approved power spectral mask and to efficiently demonstrate the chip-to-chip transmission feasibility at such high data rate is a challenging task. Some implementation challenges that were encountered and rectified are explained in the section below. 


\subsubsection{Meeting Filter Design Specifications and Chip-to-Chip}

\section{Communication Feasibility in CDE}

MATLAB simulation environment was used to calculate the optimal tap coefficient for efficient modeling of 3.1-10 GHz UWB channel. The length of the filter was chosen to be 20 taking into consideration both symmetry of coefficients and cutoff precision. The sampling frequency of the filter was $20 \mathrm{GHz}$. The power spectral density of the filter was found to be in good accordance with the required spectral mask.

However, the transistor level implementation of such a high rate system has some limitations. One of the key requirements of the system is to achieve low energy per bit transmission at a high data rate. In order to achieve the filtering of the serial data bit stream it is essential that the sampling should be done at $20 \mathrm{GHz}$, which will result in the usage of extremely high power buffers and transmitter drivers to properly filter and transmit the data according to the system specifications. This will result in very high energy consumed per bit and also a significant increase in some of the transistor sizes which will enhance the parasitic capacitance hence making the circuit extremely power and area inefficient.

Also, this would have required bigger size inductors to be used for chip-to-chip transmission at $20 \mathrm{GHz}$ which will acquire more area on chip once fabricated.

In order to overcome these limitations, a new approach was adopted which involved scaling down the bandwidth to half of its original value so that the FCC approved spectral 
mask was implemented in the range of $1.55 \mathrm{GHz}$ to $5 \mathrm{GHz}$. The entire bandpass filter was successfully implemented in CMOS 90nm technology.

\subsection{Channel Modeling in MATLAB}

The new channel has a bandwidth of $3.5 \mathrm{GHz}$ with cut off frequencies of $1.55 \mathrm{GHz}$ and 5 GHz. This channel is also modeled using MATLAB. The filter has 20 taps with sampling frequency of $10 \mathrm{GHz}$.

The optimized filter coefficients and the respective frequency spectrum is shown in figure

\section{1.}

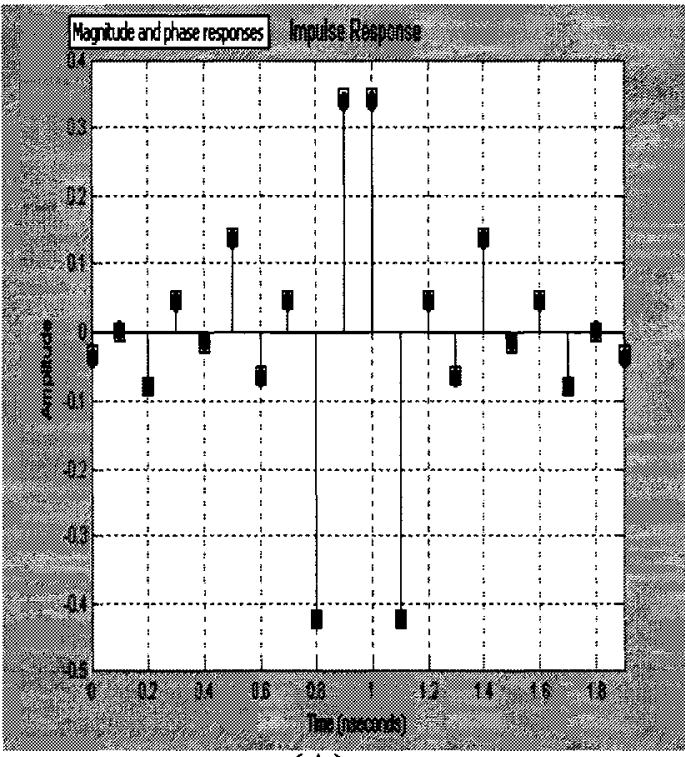

(A)

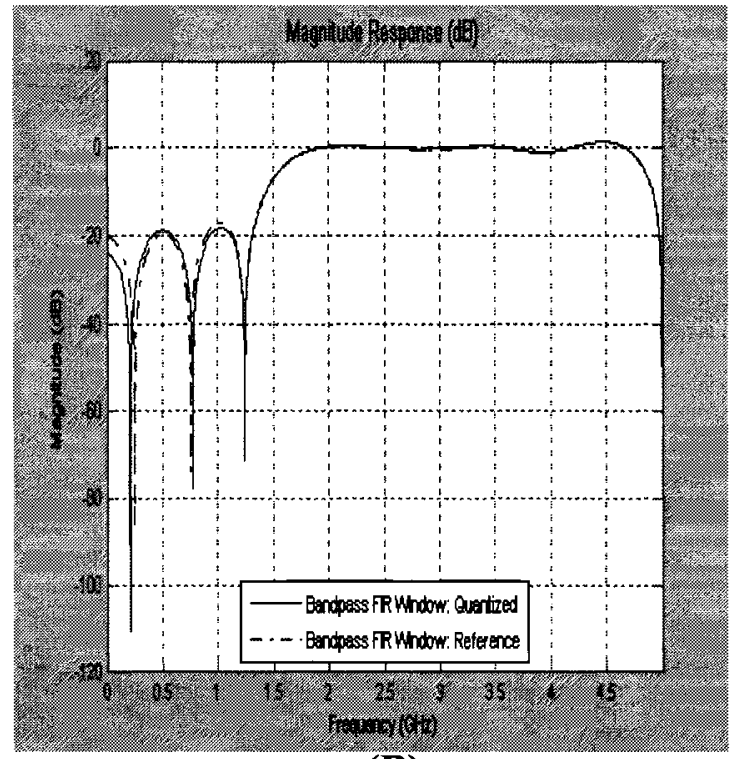

(B)

FIGURE 4.1 UWB Channel Modeling In MATLAB

UWB Ultra-Low-Power Short Distance FIR Spectrally Shaped NRZ Transmission 


\subsection{UWB Filter Implementation in $C D E$}

The simple FIR filter implementation requires basic building blocks: a delay module, multiplier unit and a summing node.The filter was implemented in CMOS 90nm technology using shift register, XOR gates and transmitter driver. All of the above filter blocks were implemented using CML structure because they are proven to be most efficient in both power and speed requirements according to many research papers.

\subsubsection{Shift Register}

A shift register consisting of a chain of $\mathrm{D}$ flip flops is used for introducing the required delay in the FIR filter. Each D flip flop consists of two D- latches arranged in a masterslave configuration and has CML structure. Figure 4.2 shows the D-Flip flop structure. The shift register has $20 \mathrm{D}$ flip flops and the clock rate is $10 \mathrm{GHz}$. The clock signal is applied after passing through a series of buffers. This shift register is shown in figure 4.3. 


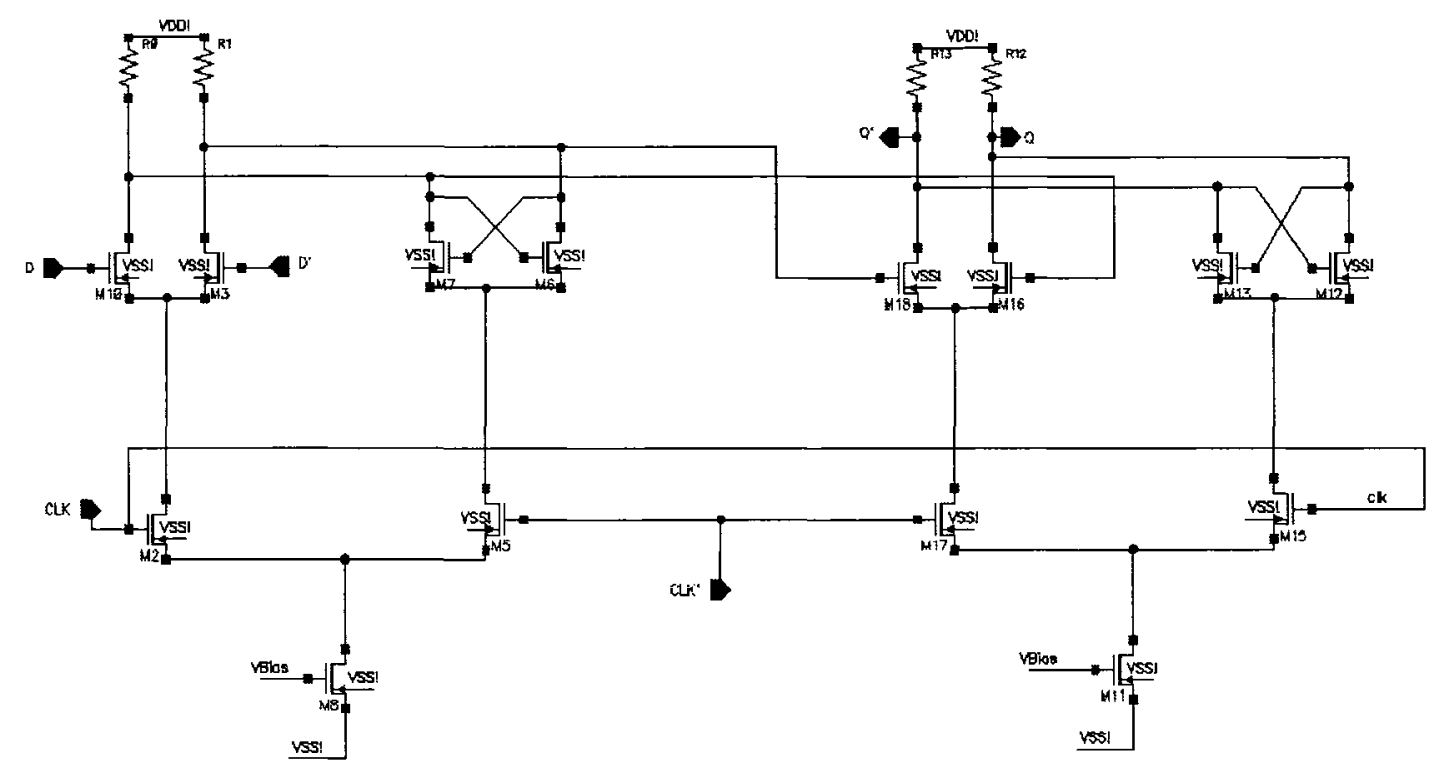

FIGURE 4.2 Schematic OF D Flip Flop
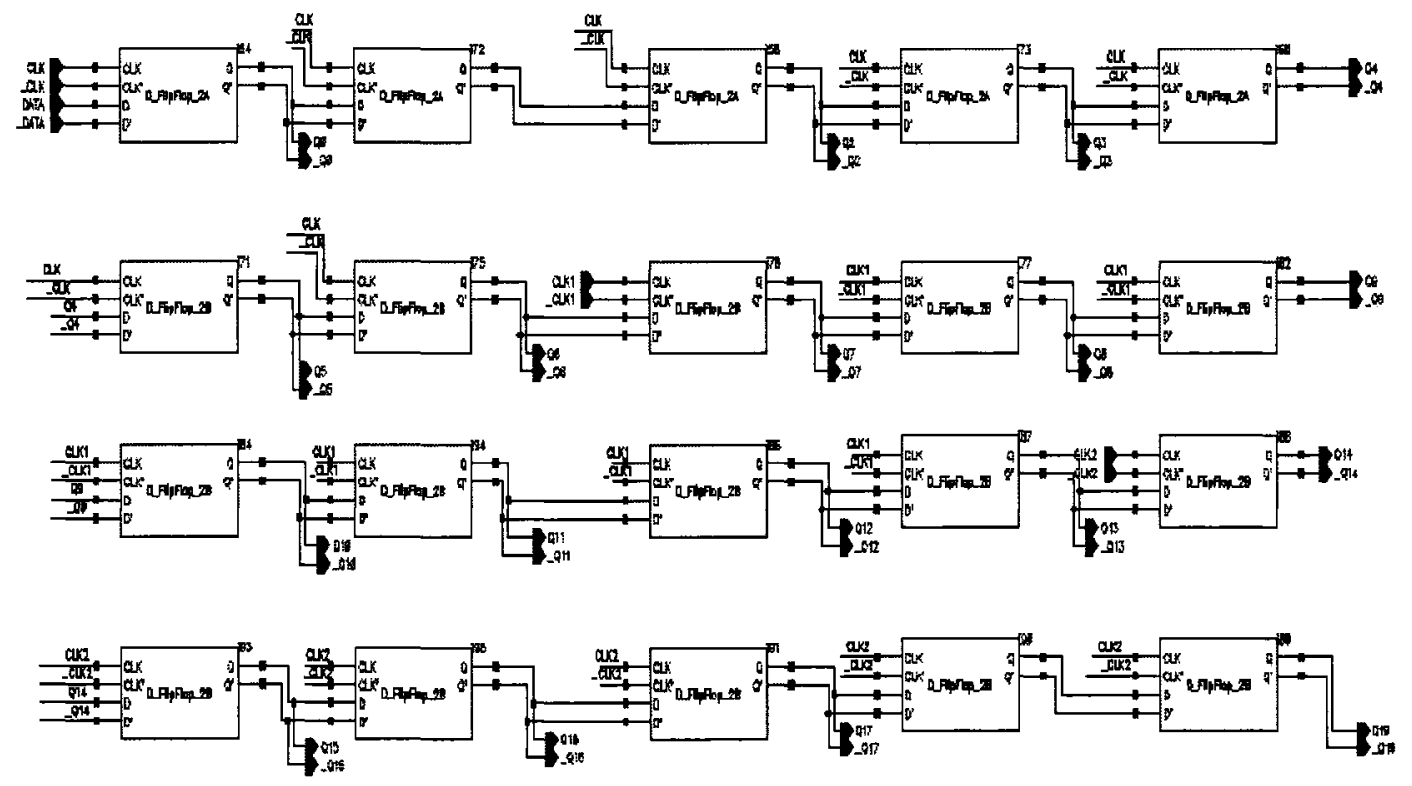

FIGURE 4.3 Shift Register - Length 20 
The output of each DFF is directly applied to the input of next DFF and is also applied to XOR gate. A pseudo random bit sequence (PRBS) consisting of 16 bits is generated at 10 Gbits/sec. Figure 4.4 shows the bit sequence passing through the shift register.

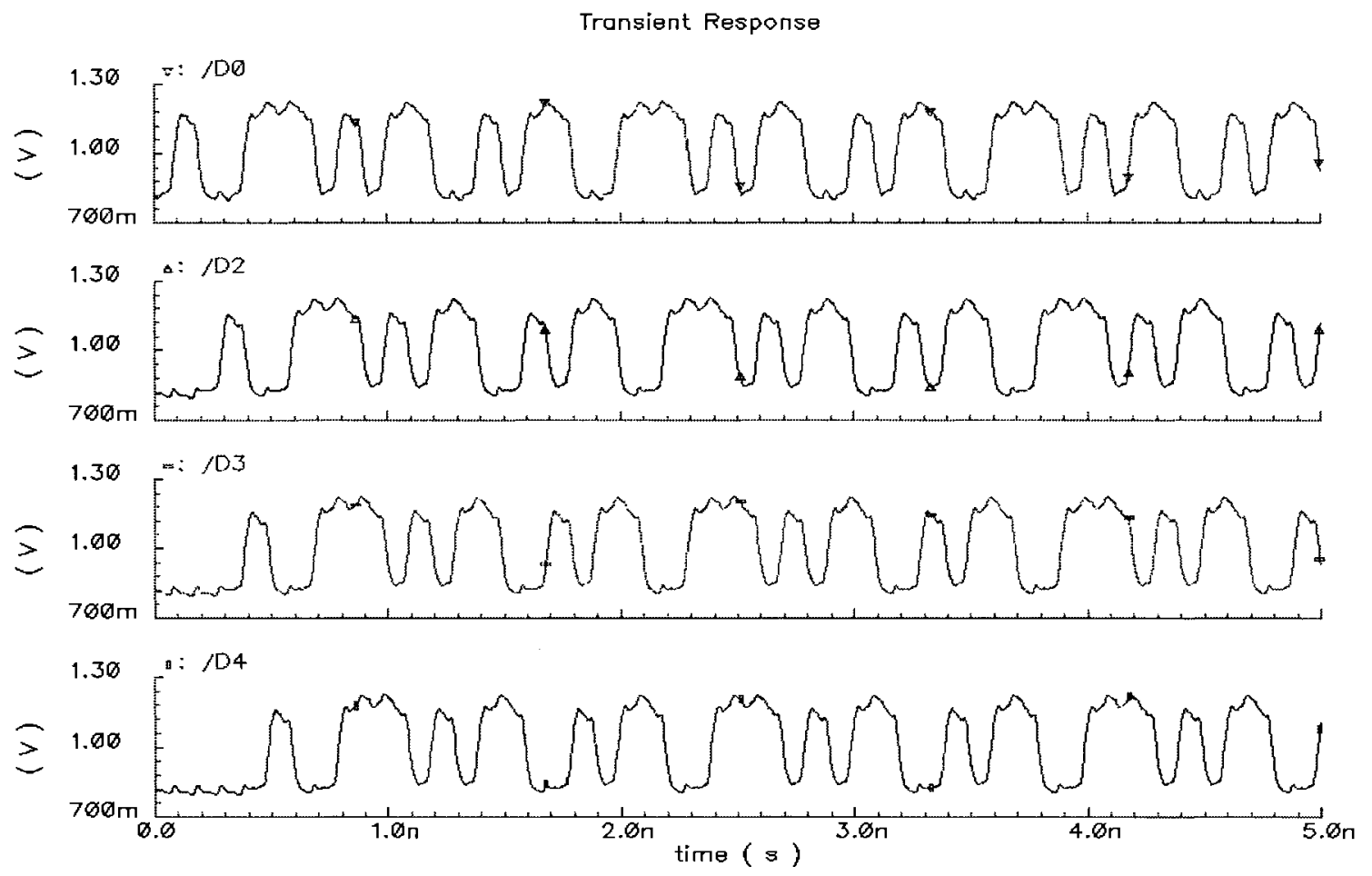

FIGURE 4.4 Data Sequence Through Shift Register

Table 4.1 lists all the specifications of the shift register.

TABLE 4. 1 Shift register specifications

\begin{tabular}{||l|c|}
\hline Width of clock transistors & $10 \mu \mathrm{m}$ \\
\hline Width of data transistors & $10 \mu \mathrm{m}$ \\
\hline Biasing current & $1 \mathrm{mAmps}$ \\
\hline Data swing & $400 \mathrm{mV}$ \\
\hline Data rise/fall time & $10 \mathrm{psec}$ \\
\hline
\end{tabular}




\subsubsection{XOR Gates}

XOR gates are used for introducing sign control in the circuit. Each data bit and its complement after passing through the DFF goes through two input XOR gate. The other input is fed by a fixed polarity signal depending upon the coefficient of that tap. The XOR gates are included for system flexibility, at any time a change in coefficient value and accordingly the change in coefficient polarity can be introduced in the system easily. Depending upon the combination of signs, the XOR gate generates a positive and negative output which is then forwarded to the driver stage. If $A$ is one input of a XOR gate and B is another input and Q is the output, then the truth table of XOR gate will be as shown in table 4.2.

TABLE 4. 2 Truth Table of XOR gate

\begin{tabular}{|c|c|c|}
\hline $\boldsymbol{A}$ & $\boldsymbol{B}$ & $\boldsymbol{Q}$ \\
\hline 0 & 0 & 0 \\
\hline 0 & 1 & 1 \\
\hline 1 & 0 & 1 \\
\hline 1 & 1 & 0 \\
\hline
\end{tabular}

As evident from the truth table above, any negative polarity whether it belongs to the data or the coefficient will generate a negative signal at the output. The CML based XOR gate is shown in figure 4.5 and the specifications are listed in table 4.3. 


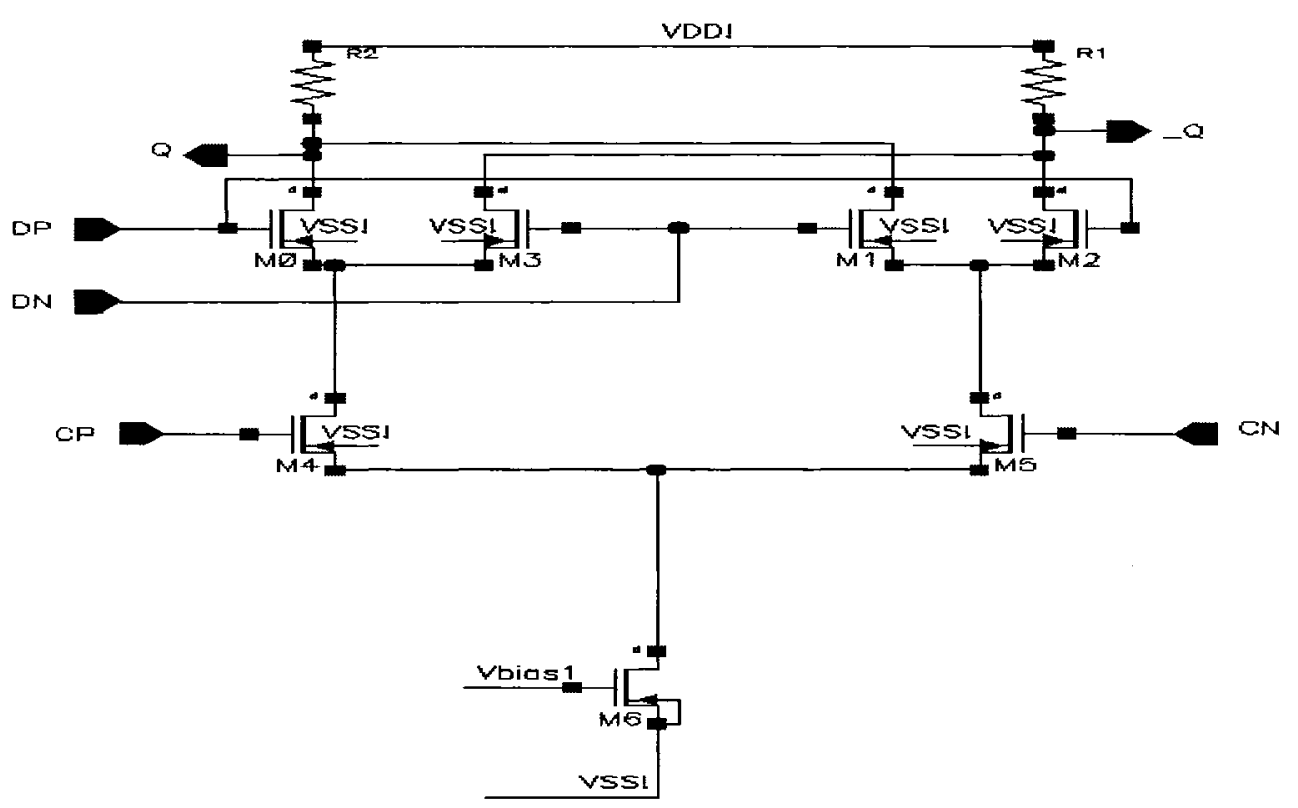

FIGURE 4.5 XOR Gate For Polarity Control

TABLE 4. 3 XOR gate specifications

\begin{tabular}{||c|c|}
\hline \hline Width of coefficient transistors & $10 \mu \mathrm{m}$ \\
\hline Width of data transistors & $10 \mu \mathrm{m}$ \\
\hline Biasing current & $1 \mathrm{mAmps}$ \\
\hline Data swing & $400 \mathrm{mV}$ \\
\hline Data rise/fall time & $10 \mathrm{psec}$ \\
\hline
\end{tabular}

4.4.3 Transmitter Driver

The transmitter driver is a CML based structure which is most significant structure for filter implementation. It executes the desired multiplication and summing functions. The mathematical expression for a FIR filter can be written as: 


$$
Y(n)=\sum_{K=-N}^{N} C_{K} \cdot X(n-K)
$$

where,

$$
\begin{aligned}
& \text { where, } \\
& \qquad \begin{array}{l}
C_{K}=\text { Coefficient of each tap } \\
K=\text { Tap number } \\
\text { and } \\
n=\text { Time instant }
\end{array}
\end{aligned}
$$

Each driver stage has two inputs for data and its complement. The outputs from XOR gates are applied on these inputs and depending on the polarity, a relevant current according to the tap value is added or subtracted at the summing nodes. Two load resistors are attached on the summing node to provide the necessary voltage drop. The tap coefficients are the normalized current values defined by using external current mirrors. Each node is normalized according to the following equation:

$$
C_{K}=\frac{I_{K}}{I_{o}}
$$

where,

$$
\begin{aligned}
& C_{K}=\text { Tap Coefficient } \\
& I_{K}=\text { Current through the specified tap } \\
& I_{o}=\text { Current through the main tap }
\end{aligned}
$$$$
\text { and }
$$

Figure 4.6 shows a three tap CML filter structure. 


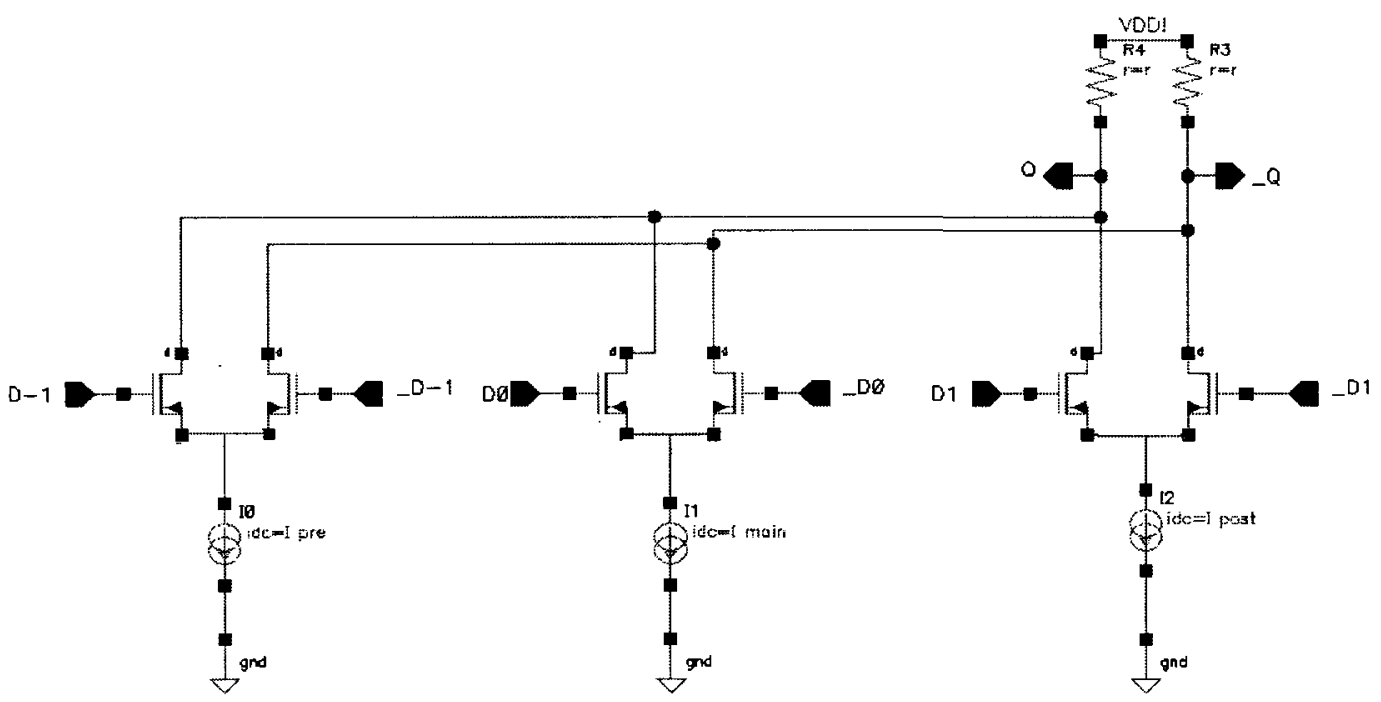

FIGURE 4.6 Three Tap Filter Shown With Pre, Main \& Post Tap Currents

The above CML structure is also referred to as a digital-to-analog converter or DAC in literature since it accepts serial data bits and at each time instant produces a voltage level analogous to the sum of the total current flowing in the circuit. The driver used in this work is shown in figure 4.7 and specifications can be found in table 4.4

TABLE 4. 4 FIR filter specifications

\begin{tabular}{|c|c|}
\hline Width of switching transistors & $7 \mu \mathrm{m}$ \\
\hline Width of biasing transistors & $27 \mu \mathrm{m}$ \\
\hline Total biasing current & $6.744 \mathrm{mAmps}$ \\
\hline Voltage swing & $400 \mathrm{mV}$ \\
\hline
\end{tabular}




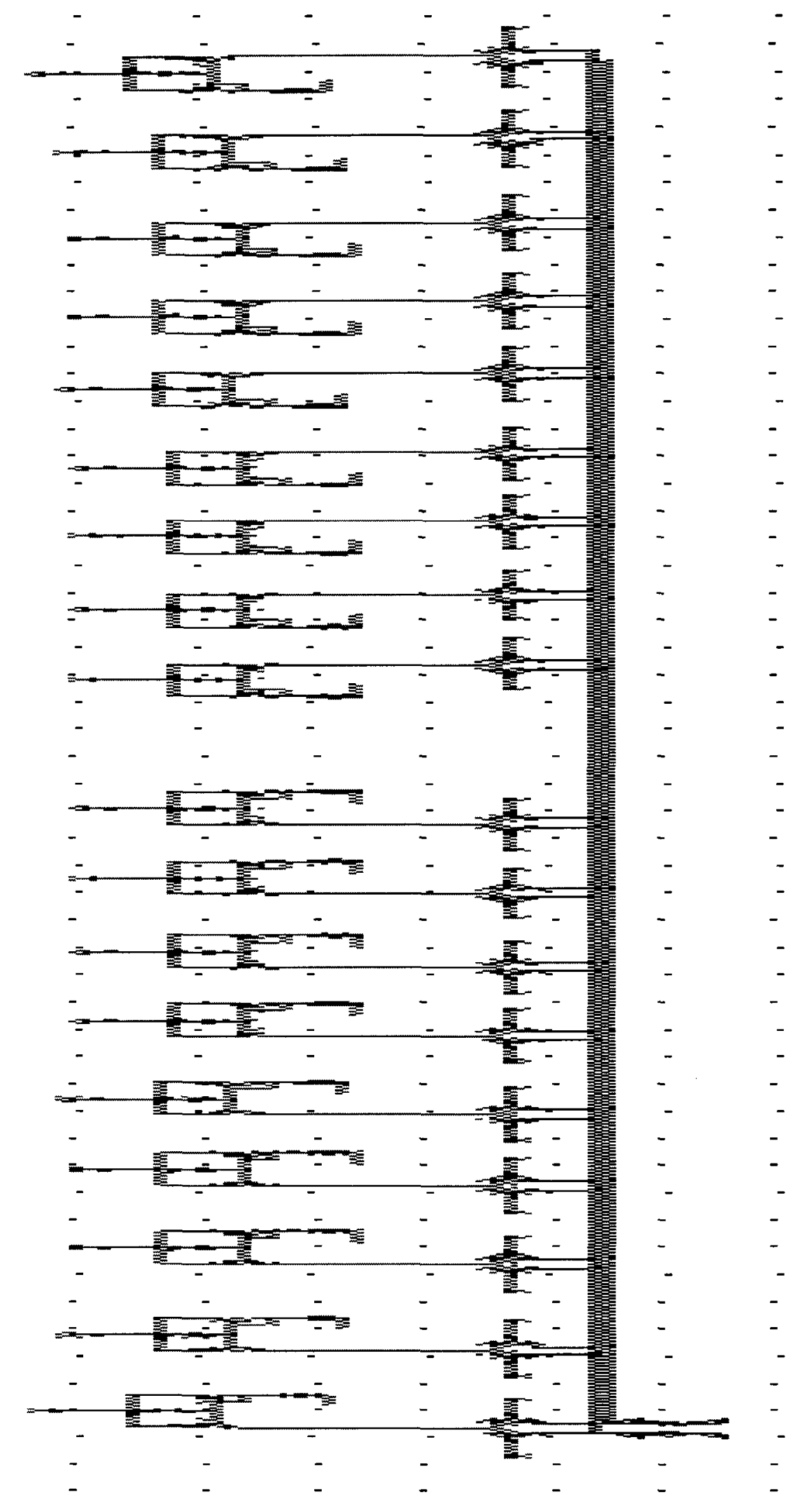

FIGURE 4.7 Transmitter Driver Consisting Of 18 Stages 


\subsection{Antenna}

Two single layer square spiral stacked inductors were used for wireless transmission and reception of data at $10 \mathrm{GHz}$. ASITIC was used for designing and extraction of results based on required specifications. Figure 4.4 shows the layout of this stacked inductor configuration. The typical spacing between the spiral inductors are $10-20 \mu \mathrm{m}$ with minimum spacing and the hollow area in the center of inductor improves the quality factor of the inductor.

The inductors are perfectly aligned with each other since it is essential for flawless data transmission. The thickness of inductors are $12 \mu \mathrm{m}$ with inner spacing of $50 \mu \mathrm{m}$ with each inductor having $3 \mathrm{nH}$ inductance. The top most metal layer was chosen for the transmitting inductor whereas the receiving inductor was designed on the 6 th metal layer. The coupling coefficient was found to be 0.95 which defines excellent mutual coupling. Figure 4.8 shows the stacked inductors.

\subsection{Receiver}

The receiver structure is shown in figure 4.9. The output from the mutually coupled biased receiver inductor is fed into a buffer / amplifier stage from which the received bit stream is recovered using the behavior CDR circuit. 


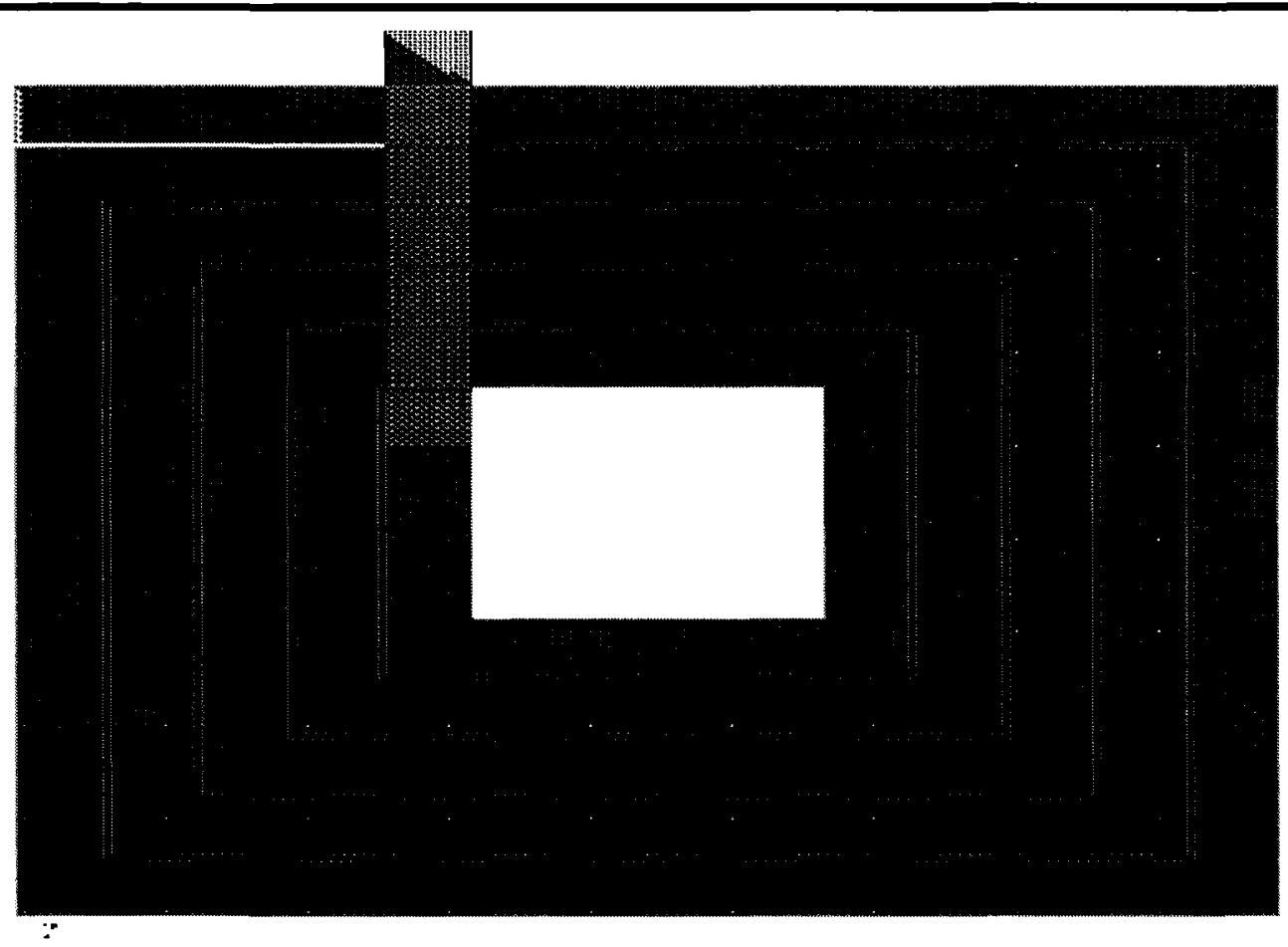

FIGURE 4.8 Stacked Inductors For Wireless Communication

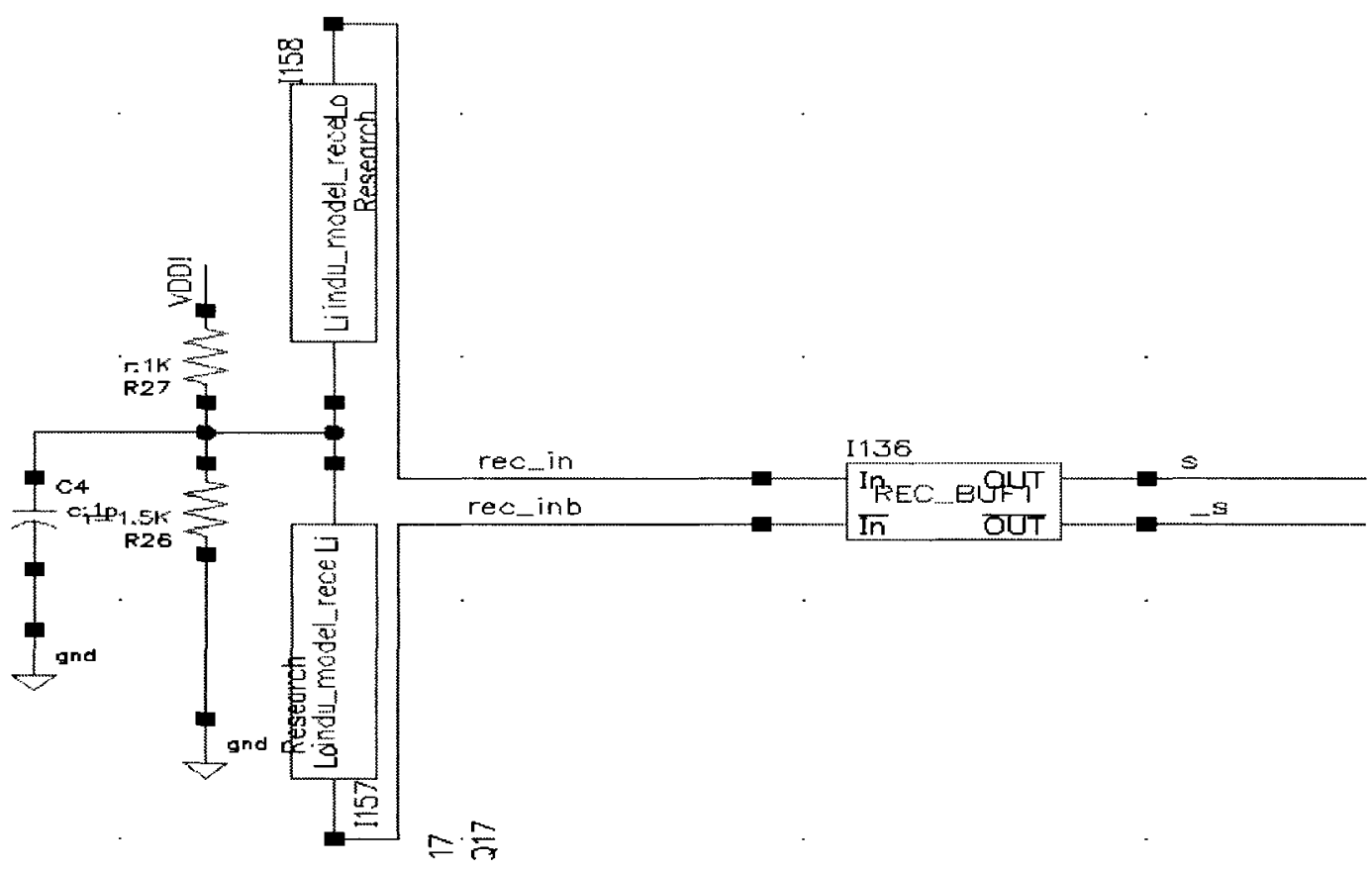

FIGURE 4.9 Receiver Structure

UWB Ultra-Low-Power Short Distance FIR Spectrally Shaped NRZ Transmission 


\subsection{Test Bench}

Both MATLAB simulation environment and CADENCE Analog SPECTRE environment were used in conjunction for entire circuit simulation. Firstly, for the spectrum verification in MATLAB noise is generated and passed through the BPF to study the received spectrum which is found to be quite in accordance with the required FCC spectral mask After that the transistor level schematic was designed in CADENCE. Figure 4.10 shows the test signals that are applied to the circuit. The test set-up consists of the basic FIR filter building block on the transmitter side that is shift register, XOR gates and the transmitter driver. Two stacked inductors that are to be fabricated on the same chip but different metal layers provide the necessary wireless communication channel between transmitter and receiver side. On receiver side, the received signal is firstly boasted by passing it through a series of buffers and then recovered by a behavior CDR circuit.

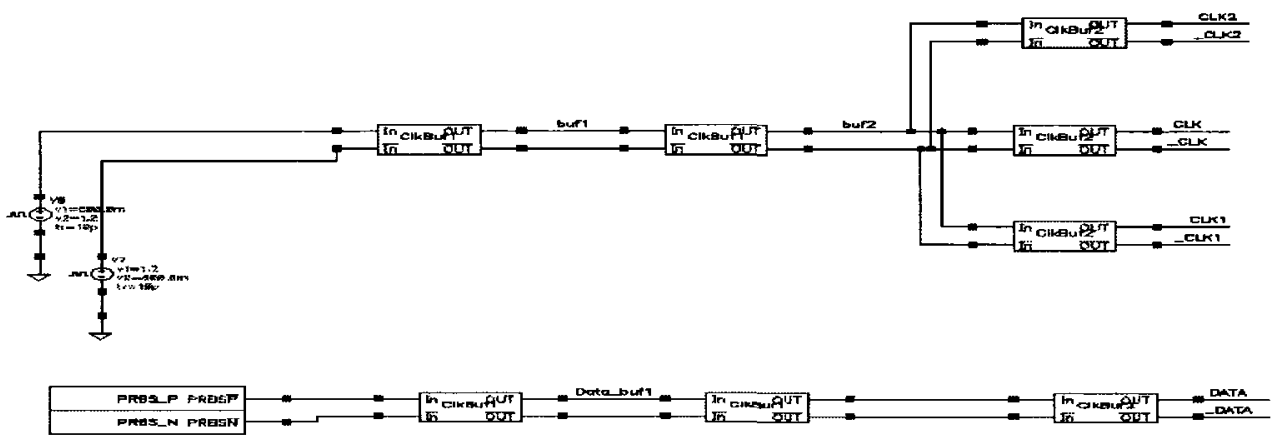

FIGURE 4.10 Data And Clock Test Signals

As evident from the above figure, a pseudorandom data sequence (PRBS) is generated at $10 \mathrm{~Gb} / \mathrm{s}$ with $10 \mathrm{ps}$ rise and fall time and is passed through a series of buffers before being 
applied to the shift register. Similarly differential clock signals are generated and passed through buffers before being applied to the circuit. Data and clock signals are then applied to the shift register.The clock is working at $10 \mathrm{GHz}$. At each clock cycle, the data bit hops to the input of next DFF and also the input of corresponding XOR gate. The other input of the XOR gate is fed through a constant voltage level high or low, depending upon the relevant coefficient. When the data bit is received on the input, the polarity multiplication function is carried out and two differential output signals (high and low) are generated at the XOR output according to the result. These signals are then applied to the switching transistor of the driver stage. The switching transistors turn on or off depending upon the applied signal on their input and a relative current flows in the circuit which is either added or subtracted on the summing nodes. At each time instant a voltage signal appears at the summing nodes, the inductors on the summing nodes transfer this voltage level to the receiver side through electromagnetic coupling. The signal on the receiver side has a very small swing and cannot be directly used for transmitted bit sequence recovery, hence it is passed through a series of buffers and then applied to a behavior CDR circuit. This circuit samples each data bit in the centre of the eye and data is recovered effectively. Figure 4.11 shows the simplified transmitter block diagram (original has differential gate structure and extracted model of inductor is used).

Figure 4.12 explains the adopted design and implementation steps with the help of a flow chart. 


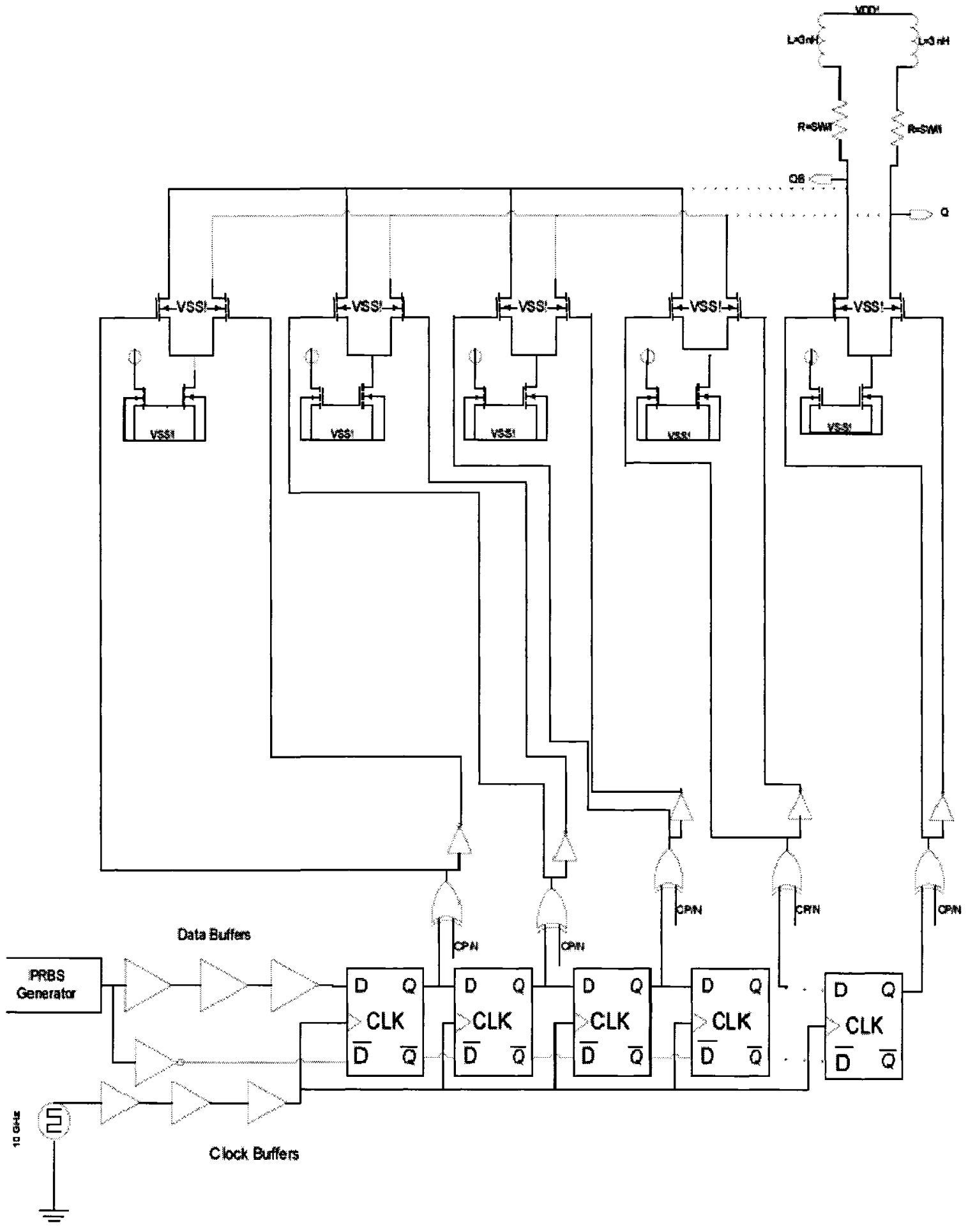

FIGURE 4.11 Transmitter Block Diagram 


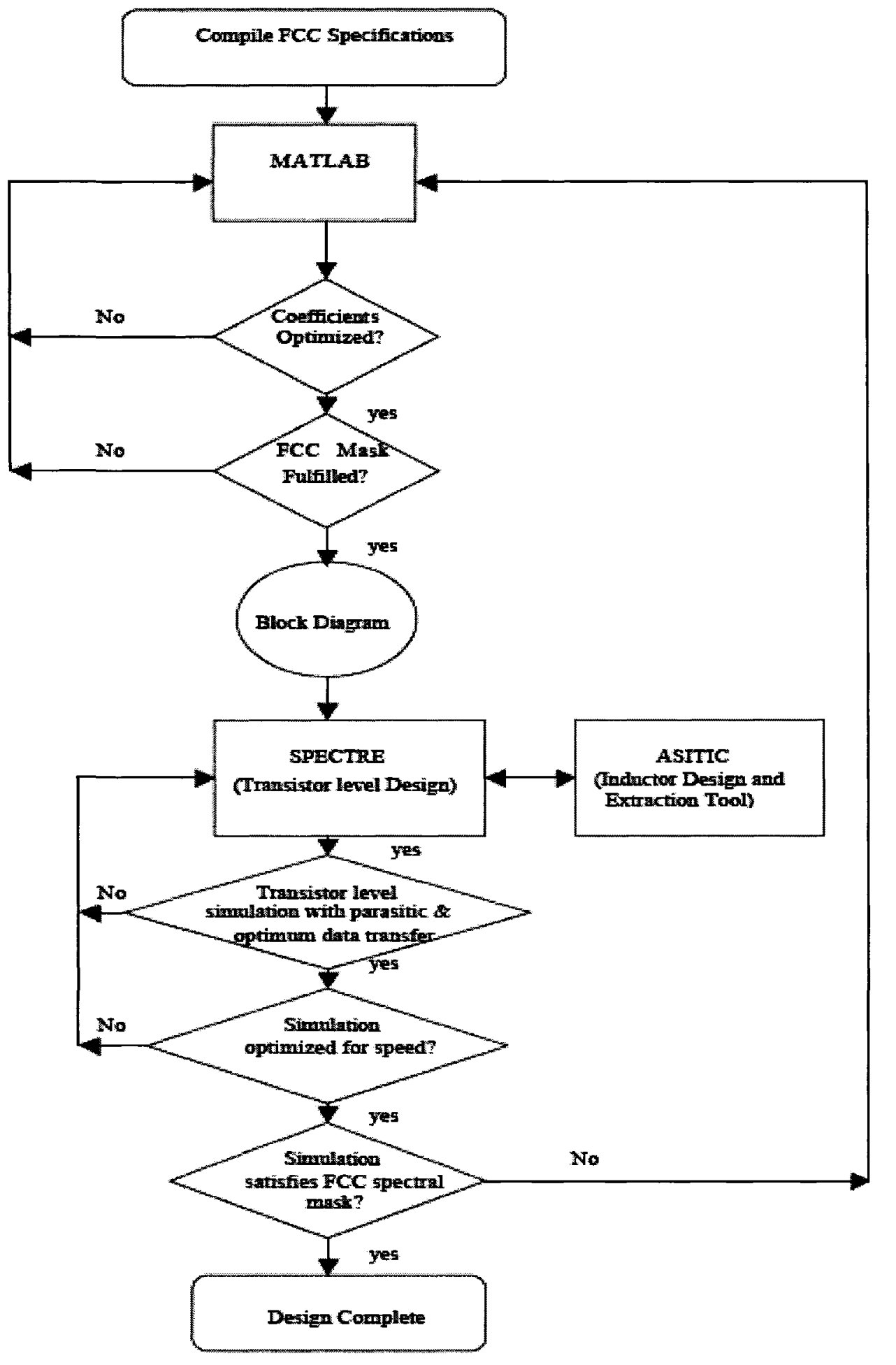

FIGURE 4.12 Flow Chart Of Design Sequence

UWB Ultra-Low-Power Short Distance FIR Spectrally Shaped NRZ Transmission 


\subsection{Performance Evaluation}

The important parameters for evaluating the performance of this work are power and energy consumption and speed of the circuit. Table 4.5 evaluates the total power consumed in the proposed transmitter excluding clock and data buffers whereas, table 4.6 compares this work to the other reported work.

\section{TABLE 4. 5 Total Power consumed in the transmitter}

\begin{tabular}{||l|l|l||}
\hline Blocks & Current $(\mathbf{m}$ Amps) & Power $(\boldsymbol{m} \boldsymbol{W})$ \\
\hline Shift register & 20 & 24 \\
\hline XOR gate & 18 & 21.6 \\
\hline Filter & 6.744 & 8 \\
\hline Total & 44.744 & 53.6 \\
\hline \hline
\end{tabular}

TABLE 4. 6 Comparison With other Reported Work

\begin{tabular}{|c|c|c|c|c|c|c|}
\hline References & Methodology & Data Rate & $\begin{array}{l}\text { Power } \\
(m W)\end{array}$ & Energy/bit & $\operatorname{Area}\left(\mathrm{mm}^{2}\right)$ & Technology \\
\hline$[9]^{(b)}$ & $I R$ & $4 \mathrm{Mb} / \mathrm{s}$ & $\begin{array}{l}20.48 @ \\
3.3 \mathrm{~V}\end{array}$ & $5.12 \mathrm{~nJ}$ & 0.16 & $0.35 \mu \mathrm{m}$ \\
\hline$[10]^{(b)}$ & $I R$ & $\begin{array}{l}500 \mathrm{MHz} \\
(P R F) \\
1 \mathrm{MHz} \\
(P R F)\end{array}$ & $\begin{array}{l}15.4 @ \\
1.8 \mathrm{~V} \\
0.675 @ \\
1.8 \mathrm{~V} \\
\end{array}$ & - & - & $0.18 \mu \mathrm{m}$ \\
\hline$[16]^{(a)}$ & $O F D M$ & $480 \mathrm{Mb} / \mathrm{s}$ & $\begin{array}{l}105 @ \\
1.5 \mathrm{~V}\end{array}$ & $218 \mathrm{pJ}$ & 1 & $130 \mathrm{~nm}$ \\
\hline$[19]^{(a)}$ & $I R$ & $1 \mathrm{Mb} / \mathrm{s}$ & $\begin{array}{l}1 @ \\
1.8 \mathrm{~V}\end{array}$ & $\ln J$ & 0.04 & $0.18 \mu \mathrm{m}$ \\
\hline$[20]^{(a)}$ & $\begin{array}{l}\text { Analog } \\
\text { signal } \\
\text { processing }\end{array}$ & $50 \mathrm{Mb} / \mathrm{s}$ & $\begin{array}{l}41.4 @ 19 \\
1.8 \mathrm{~V}\end{array}$ & $828 \mathrm{pJ}$ & $\begin{array}{l}1.085 \mathrm{x} \\
0.79\end{array}$ & $0.18 \mu \mathrm{m}$ \\
\hline
\end{tabular}


TABLE 4. 6 Comparison With other Reported Work

\begin{tabular}{|c|c|c|c|c|c|c|}
\hline References & Methodology & Data Rate & $\begin{array}{l}\text { Power } \\
(m W)\end{array}$ & Energy/bit & $\operatorname{Area}\left(\mathrm{mm}^{2}\right)$ & Technology \\
\hline$[31]^{(a)}$ & $802.15 .4 a$ & $\begin{array}{l}1 \mathrm{Mb} / \mathrm{s} @ \\
3.1 \mathrm{G} \\
1 \mathrm{Mb} / \mathrm{s} @ \\
10 G\end{array}$ & $\begin{array}{l}0.65 @ \\
1 V \\
1.4 @ \\
1 V\end{array}$ & $\begin{array}{l}0.65 \mathrm{~nJ} \\
1.4 \mathrm{~nJ}\end{array}$ & - & $90 \mathrm{~nm}$ \\
\hline$[32]^{(a)}$ & $I R$ & $1.16 \mathrm{~Gb} / \mathrm{s}$ & $\begin{array}{l}12.6 @ \\
1.8 \mathrm{~V}\end{array}$ & $10.86 p J$ & 0.1387 & $0.18 \mu \mathrm{m}$ \\
\hline$[33]^{(a)}$ & $I R$ & $1 \mathrm{~Gb} / \mathrm{s}$ & $\begin{array}{l}108 @ \\
1.8 \mathrm{~V}\end{array}$ & $108 p J$ & $4.3 \times 3.5$ & $0.18 \mu \mathrm{m}$ \\
\hline$[34]^{(a)}$ & $I R$ & $160 \mathrm{Mb} / \mathrm{s}$ & $\begin{array}{l}10 @ \\
1.2 \mathrm{~V}\end{array}$ & $62.5 p J$ & 1.56 & $130 \mathrm{~nm}$ \\
\hline$[35]^{(a)}$ & $I R$ & $100 \mathrm{Mb} / \mathrm{s}$ & $\begin{array}{l}180 @ \\
3.3 \mathrm{~V}\end{array}$ & $1.8 n J$ & $1.5 \times 1.3$ & $0.35 \mu \mathrm{m}$ \\
\hline$[36]^{(a)}$ & $\begin{array}{l}\text { Phased } \\
\text { Array }\end{array}$ & $480 \mathrm{Mb} / \mathrm{s}$ & $\begin{array}{l}\text { Tx sing. } \\
397 \\
T x \text { dual. } \\
435\end{array}$ & $\begin{array}{l}0.827 n J \\
0.906 n J\end{array}$ & $4.2 \times 3.8$ & $0.18 \mu \mathrm{m}$ \\
\hline$[37]^{(a)}$ & $I R$ & $\begin{array}{l}10 \mathrm{Mb} / \mathrm{s} \\
@ 10 \mathrm{~m} \\
250 \mathrm{~kb} / \mathrm{s} \\
@ 30 \mathrm{~m}\end{array}$ & $\begin{array}{l}29.7 @ \\
2.2 \mathrm{~V}\end{array}$ & $\begin{array}{l}2.97 n J \\
118 n J\end{array}$ & 0.4 & $0.18 \mu \mathrm{m}$ \\
\hline$[38]^{(a)}$ & $I R$ & $\begin{array}{l}1.8 \\
\text { Gpulses/s }\end{array}$ & $\begin{array}{l}227 @ \\
1 V\end{array}$ & $\begin{array}{l}126 \mathrm{pJ} \\
\text { pulse }\end{array}$ & 2.83 & $90 \mathrm{~nm}$ \\
\hline $\begin{array}{l}\text { This } \\
\text { work }^{(b)}\end{array}$ & Serial Data & $10 \mathrm{~Gb} / \mathrm{s}$ & $\begin{array}{l}53.6 @ \\
1.2 \mathrm{~V}\end{array}$ & $5.36 p J$ & $0.1 \times 0.13$ & $90 \mathrm{~nm}$ \\
\hline
\end{tabular}

Only CMOS Technologies are discussed

The above table lists the comparison between various simulated and measured works. It can be gathered from the table that this work reports lowest energy / bit with highest data rate whereas power consumption and layout area is comparable to the other reported works. 


\subsection{Chapter Overview}

This chapter present the simulation results and performs a thorough analysis of the obtained results.

\subsection{Optimal Tap Coefficients and Modeled UWB Channel}

The optimal tap coefficients for the bandpass filter were obtained through MATLAB. Figure 5.1 shows the optimized tap coefficients and the relative signal bandwidth (repeated from section 4.3). The half scale bandwidth of the bandpass filter is from $1.55 \mathrm{GHz}$ to 5 $\mathrm{GHz}$ and the coefficients also correspond to the half scale filter. If the full scale filter is to be employed, new optimized filter coefficients will have to be calculated using MATLAB. In transistor level circuit implementation, the use of XOR gates provide the necessary flexibility to adapt to a different set of filter coefficients. 

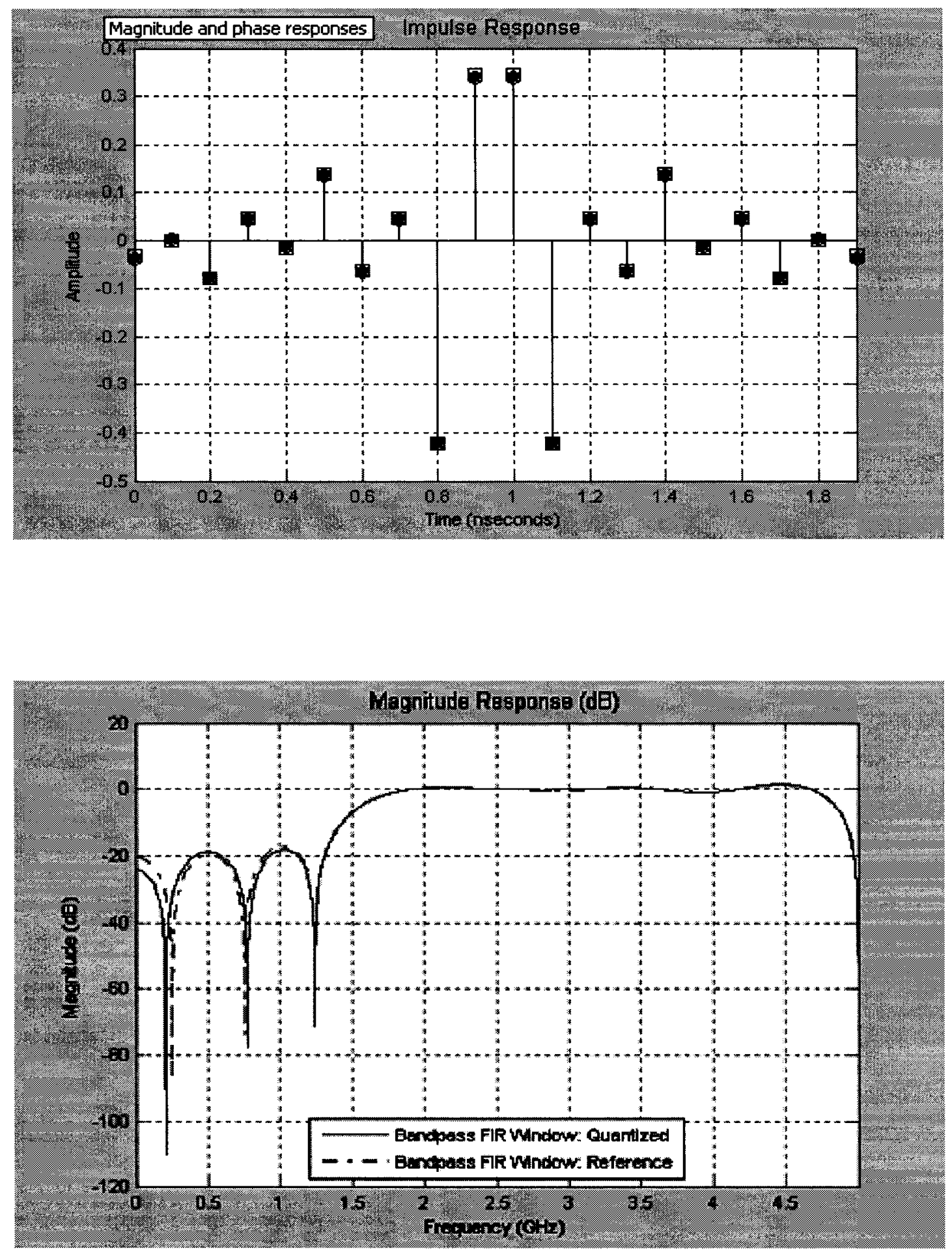

FIGURE 5.1 UWB Channel Modeling in MATLAB 
Figure $5.2 \& 5.3$ shows the power spectrum of the designed filter obtained by passing randomly generated noise signal through the filter, the obtained spectrum almost satisfies the FCC spectral mask regulations.

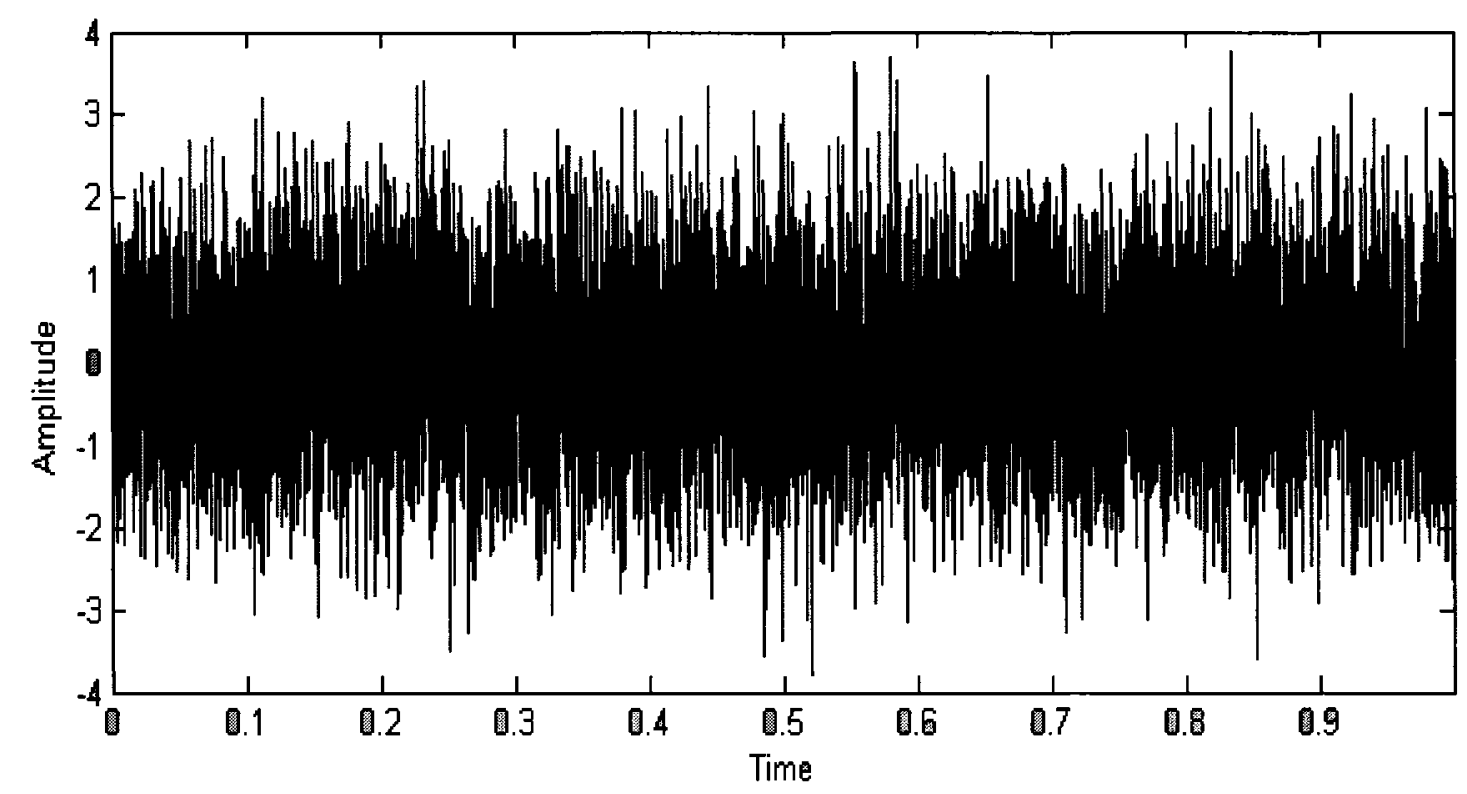

FIGURE 5.2 Generated Random Noise Signal

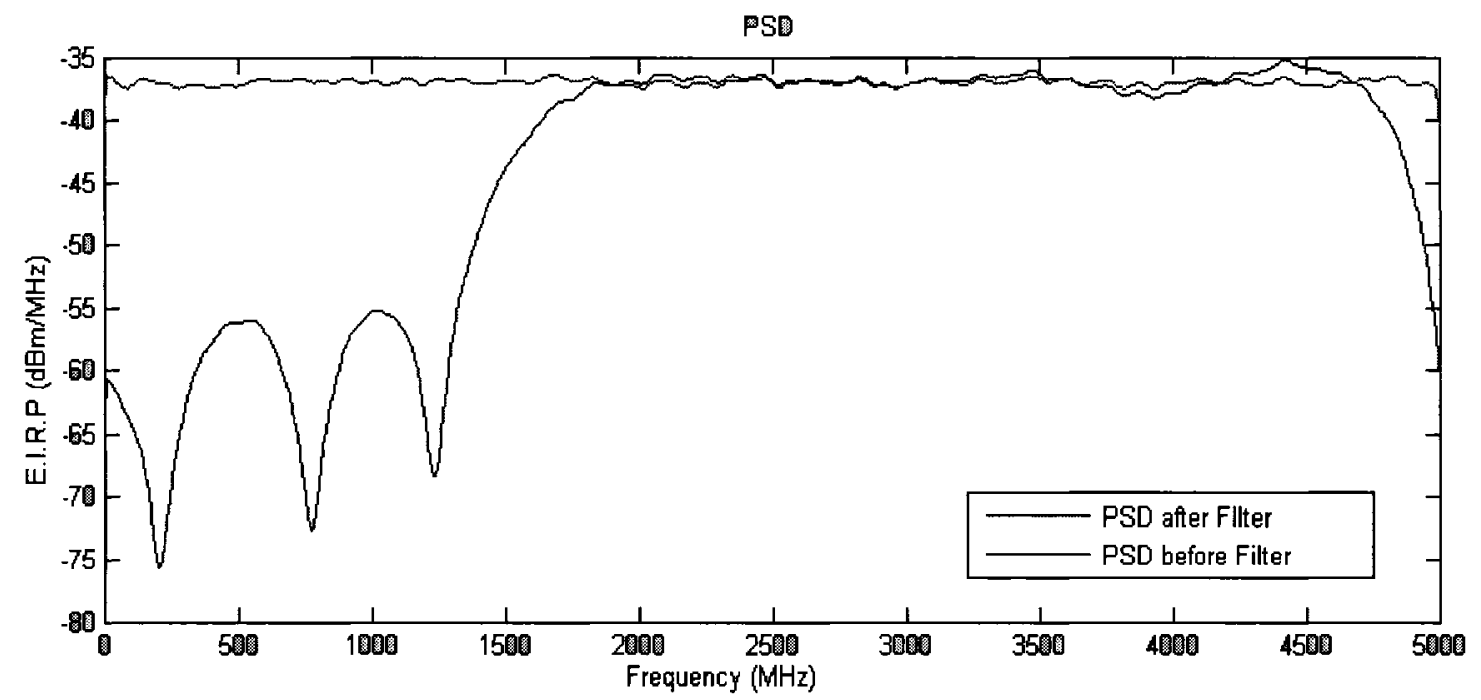

FIGURE 5.3 Power Spectral Density Comparison 


\subsection{Transmitted Data Stream}

A transistor level model for the transmitter was carried out in CMOS 90nm technology. The supply voltage of the transmitter is basically $1.2 \mathrm{~V}$, with all the buffers also operating at the same supply voltage. Figure 5.4 shows the simulated waveform The differential signal was measured at transmitter output. The figure also shows the bit pattern corresponding to each signal. The transmitted signal has a voltage swing of almost $430 \mathrm{mV}$ with signal variation between 1.1 and $600 \mathrm{mV}$. The inductor has a value of $3 \mathrm{nH}$ and produces sharp detectable edge transitions at the end of each data bit. A behavior model of comparator circuit working at $10 \mathrm{GHz}$ was used to decode the transmitted bits. The transmitted bit pattern is 1001110100100100 and is repeated after every 16 bits. The total current through the transmitter is $6.744 \mathrm{~mA}$ and the total power consumed is $8 \mathrm{mWatts}$.

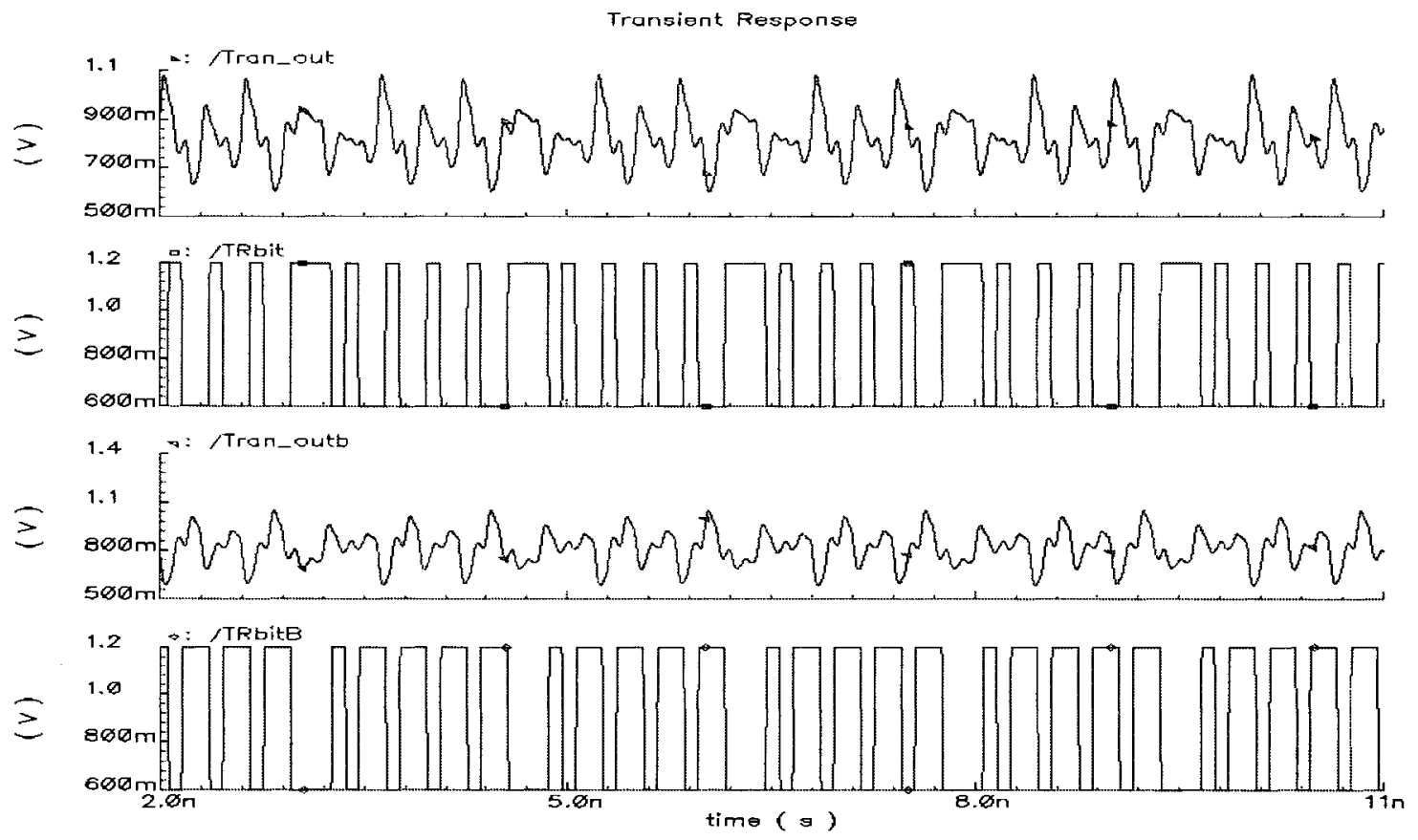

FIGURE 5.4 Transmitted Data Sequence

UWB Ultra-Low-Power Short Distance FIR Spectrally Shaped NRZ Transmission 


\subsection{Received Data Stream}

Figure 5.5 below shows the differential received data stream and the corresponding bit patterns at the output of receiver antenna. The signal swing is almost $250 \mathrm{mV}$ nearly half the value at the transmitter end. The received signal is passed through a series of buffers and then finally sent to the behavior clock and data recovery circuit for detection and decoding. The received bit stream was found to be 1001110110100100 and is repeated after the 16 bit cycle. Since the data bit rate is $10 \mathrm{~Gb} / \mathrm{s}$, the CDR is sampling at $10 \mathrm{GHz}$ and the behavior model is designed so that the data bit is always sampled in the centre.

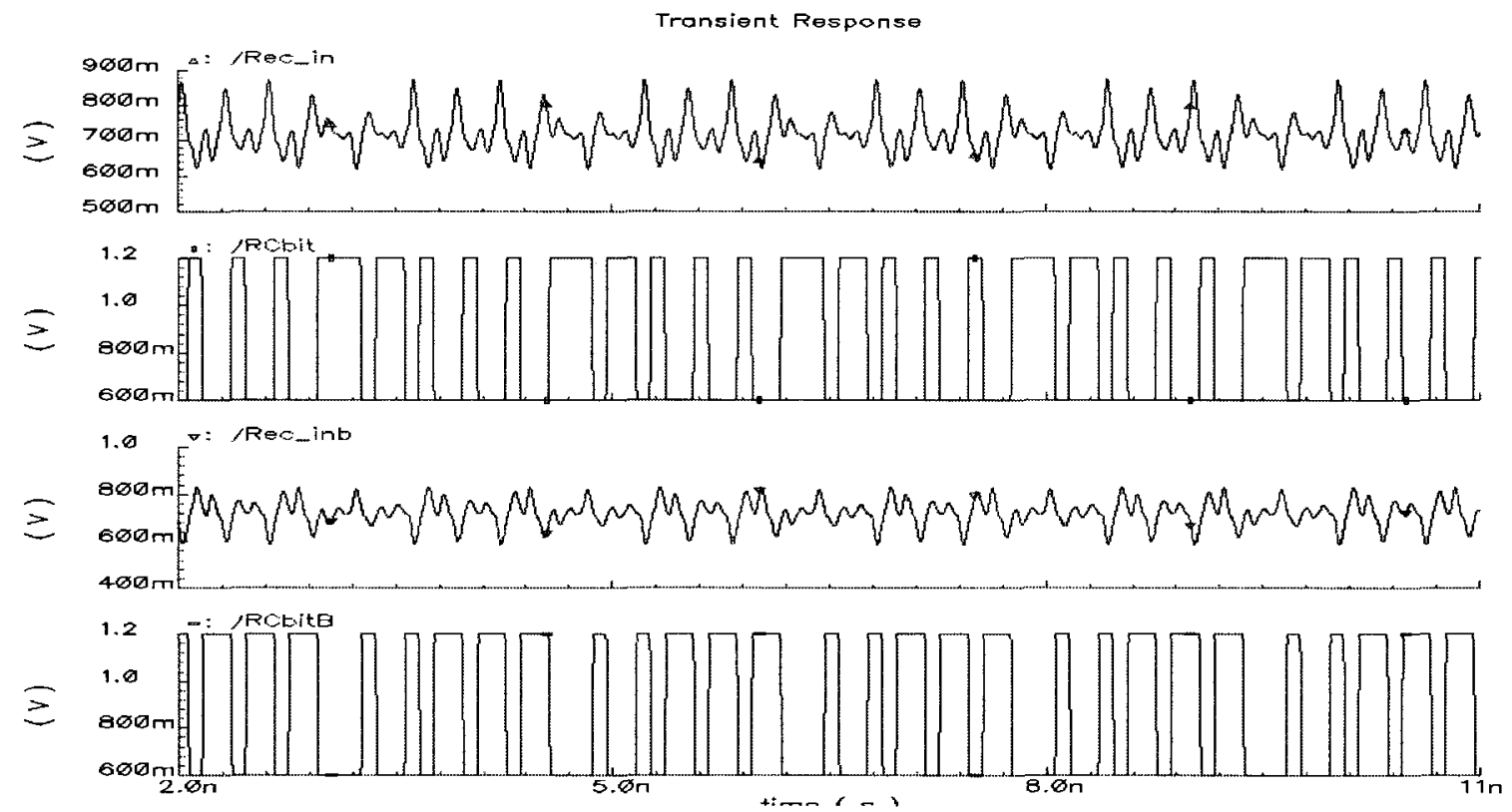

FIGURE 5.5 Received Data Sequence

\subsection{Analysis}

Figure 5.6 shows a comparison between the transmitted and received signal and recovered data stream. It can be seen that although the transmitted and received data signals are in UWB Ultra-Low-Power Short Distance FIR Spectrally Shaped NRZ Transmission 
complete concordance with each other, one of the received bits is incorrectly recovered.

The reason may be the cutoff of low frequency components in the signal level at the receiver end. Applying some equalization technique at the receiver end can certainly take care of this problem.

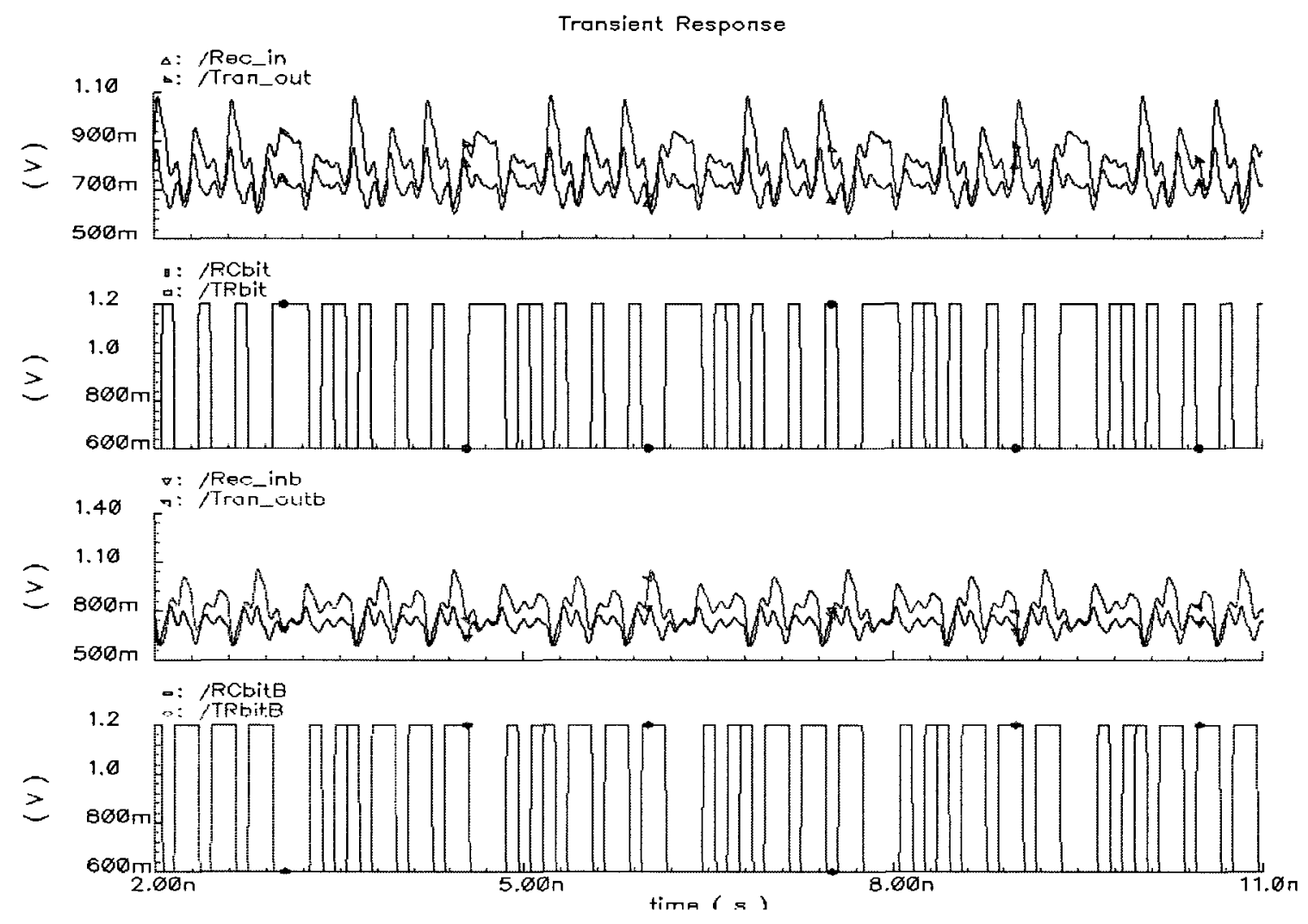

FIGURE 5.6 Comparison Between Transmitted and Received Data Sequences

In order to verify that the transmitted sequence has been filtered according to the FCC spectral mask, the transmitter was simulated for a period of $100 \mathrm{~ns}$ and the resultant power spectrum is plotted in figure 5.7. Since the simulated data has a duration of $100 \mathrm{~ns}$, the spectrum resolution is of $10 \mathrm{MHz}$. As such, for comparison with the FCC mask, the values in the figure must decrease by $10 \log (10)=10 \mathrm{~dB}$, because the FCC spectral mask is 
defined using resolution of $1 \mathrm{MHz}$. Although, the designed filter compromises somewhat on the FCC spectral mask, it overcomes that by offering simplicity in implementation and low power dissipation.

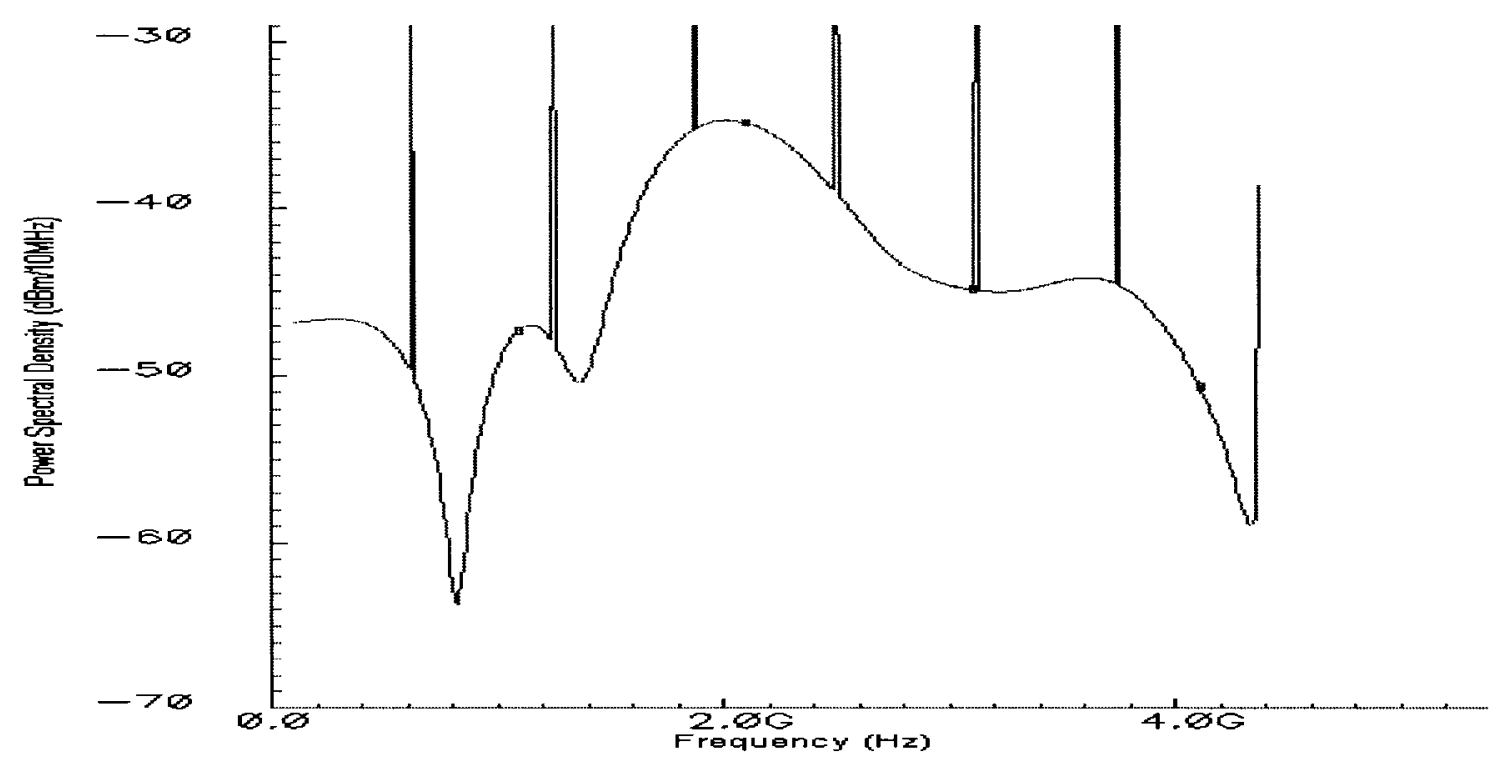

FIGURE 5.7 Power Spectrum of Transmitted Sequence in SPECTRE

The power attenuation at higher frequencies is related to the non-idealities of the implemented filter transfer function and can be taken care of by changing the tap delay thus increasing the sampling frequency or by increasing the number of taps. 


\subsection{Chapter Overview}

This chapter provides a short summary \& conclusions drawn from the thesis work. The chapter also lists a few recommendations for future work

\subsection{Synopsis \& Conclusions}

This thesis provides a detail review of existing wireless techniques with emphasis on ultra low power solutions for ultra wideband communications. Based on the expertise of our research group, NRZ signaling in ultra wideband has been selected as the exploration target. The thesis successfully establishes high speed chip-to-chip transmission with a small circuit and power dissipation. The beauty of the work lies in the idea that very simple building blocks that are extensively used in communication systems are used for the spectral modeling of ultra wideband which in essence is a challenging task. Although collective work has been reported in the literature employing different methodologies and 
circuit structures to accomplish UWB communication, to the best of my knowledge, serial data stream has never been reported to be used for UWB communication. A contributing publication is finalized. A quick review of the thesis work and the details of contribution is as follows: A high speed NRZ serial data stream is generated and filtered through an efficient CML based FIR structure. The FIR filter structure also incorporates XOR gates for coefficient polarity control hence allowing room for reconfiguration and bandwidth flexibility. Optimum coefficients for the bandpass filter were obtained from MATLAB while the entire circuit was designed and simulated in CDE (CADENCE design environment). The behavior modeling of the circuit was done using Verilog-A. ASITIC was used to extract the antenna inductors that are arranged in a stacked configuration for transmission / reception of data stream. The inductors are of $3 \mathrm{nH}$ and have a coupling coefficient of 0.95 .

The summary of contributions is as follows:

- To my knowledge extent this is the first work which reports spectrally shaped filtered transmission of NRZ serial data at such high data rate and low transmission power targeting UWB channel. The best comparison in terms of circuit structure can be found in [38] where an FIR filter is used to generate UWB pulses. However, the power consumption of only the pulse generator was $129 \mathrm{~mW}$ with a speed of $1.8 \mathrm{Gpulses} / \mathrm{s}$.

- Wireless transmission of a filtered serial data bit stream through inductive coupling is achieved with lowest energy consumed per bit as compared to other reported work 
listed in Table 3.1. in accordance with FCC spectral mask targeting the half-scaled ultra wideband channel (1.5 GHZ - $5 \mathrm{GHz})$.

- Area efficient stacked inductors are used for chip-to-chip data transmission. The layout area of inductors was found to be $0.218 \times 0.218 \mathrm{~mm}^{2}$, almost half the area of flip-chip bonding pad which is $0.4 \times 0.4 \mathrm{~mm}^{2}$, with excellent mutual coupling coefficient of 0.95. The entire transmitter structure has been designed in 90nm CMOS IC technology with a total area of $0.1 \mathrm{~mm} \times 0.13 \mathrm{~mm}$ and $53.6 \mathrm{~mW}$ power consumption at $1.2 \mathrm{~V}$ supply.

- High speed Chip-to-Chip wireless communication feasibility is conducted and the data is transferred at a rate of $10 \mathrm{Gbps}$.

\subsection{Recommendations For Future Work}

Some potential areas for future work involves:

- Establishing the same circuit topology in a more advance technology such as $65 \mathrm{nms}$ for successful simulation at full scale bandwidth with actual signal.

- Delineate complete road map for fabrication and testing. 
APPENDIX A

Spectral Emission Limits Tables

TABLE A. I FCC Spectral Emission Limits For Indoor Communication Devices

\begin{tabular}{|c|c|}
\hline Frequency $(\mathrm{GHz})$ & E.I.R.P $(\mathrm{dBm} / \mathrm{MHz})$ \\
\hline $0.96-1.6$ & -75.3 \\
\hline $1.6-1.99$ & -53.3 \\
\hline $1.99-3.1$ & -51.3 \\
\hline $3.1-10.6$ & -41.3 \\
\hline Above 10.6 & -51.3 \\
\hline
\end{tabular}

TABLE A. 2 FCC Spectral Emission Limits For Outdoor Communication Devices

\begin{tabular}{||c|c|}
\hline Frequency $(\mathrm{GHz})$ & E.I.R.P $(\mathrm{dBm} / \mathrm{MHz})$ \\
\hline $0.96-1.6$ & -75.3 \\
\hline $1.6-1.99$ & -63.3 \\
\hline $1.99-3.1$ & -61.3 \\
\hline $3.1-10.6$ & -41.3 \\
\hline Above 10.6 & -61.3 \\
\hline
\end{tabular}


TABLE A. 3 FCC Spectral Emission Limits For Imaging Systems Above 3.1 GHz

\begin{tabular}{|c|c|}
\hline Frequency $(\mathrm{GHz})$ & E.I.R.P $(\mathrm{dBm} / \mathrm{MHz})$ \\
\hline $0.96-1.6$ & -63.3 \\
\hline $1.6-1.99$ & -53.3 \\
\hline $1.99-3.1$ & -51.3 \\
\hline $3.1-10.6$ & -41.3 \\
\hline Above 10.6 & -51.3 \\
\hline
\end{tabular}

TABLE A. 4 FCC Spectral Emission Limits For Imaging Systems Above 1.9 GHz

\begin{tabular}{|c|c|}
\hline Frequency $(\mathrm{GHz})$ & E.I.R.P $(\mathrm{dBm} / \mathrm{MHz})$ \\
\hline $0.96-1.6$ & -53.3 \\
\hline $1.6-1.99$ & -51.3 \\
\hline $1.99-10.6$ & -41.3 \\
\hline Above 10.6 & -51.3 \\
\hline
\end{tabular}

TABLE A. 5 FCC Spectral Emission Limits For Imaging Systems Below $0.96 \mathrm{GHz}$

\begin{tabular}{|c|c|}
\hline Frequency $(\mathrm{GHz})$ & E.I.R.P $(\mathrm{dBm} / \mathrm{MHz})$ \\
\hline $0.96-1.6$ & -65.3 \\
\hline $1.6-1.99$ & -53.3 \\
\hline Above 1.99 & -51.3 \\
\hline
\end{tabular}

TABLE A. 6 FCC Spectral Emission Limits For Vehicular Radar Systems

\begin{tabular}{|c|c|}
\hline Frequency $(\mathrm{GHz})$ & E.I.R.P $(\mathrm{dBm} / \mathrm{MHz})$ \\
\hline $0.96-1.61$ & -75.3 \\
\hline $1.6-22$ & -61.3 \\
\hline $22-29$ & -41.3 \\
\hline $29-31$ & -51.3 \\
\hline Above 31 & -61.3 \\
\hline
\end{tabular}




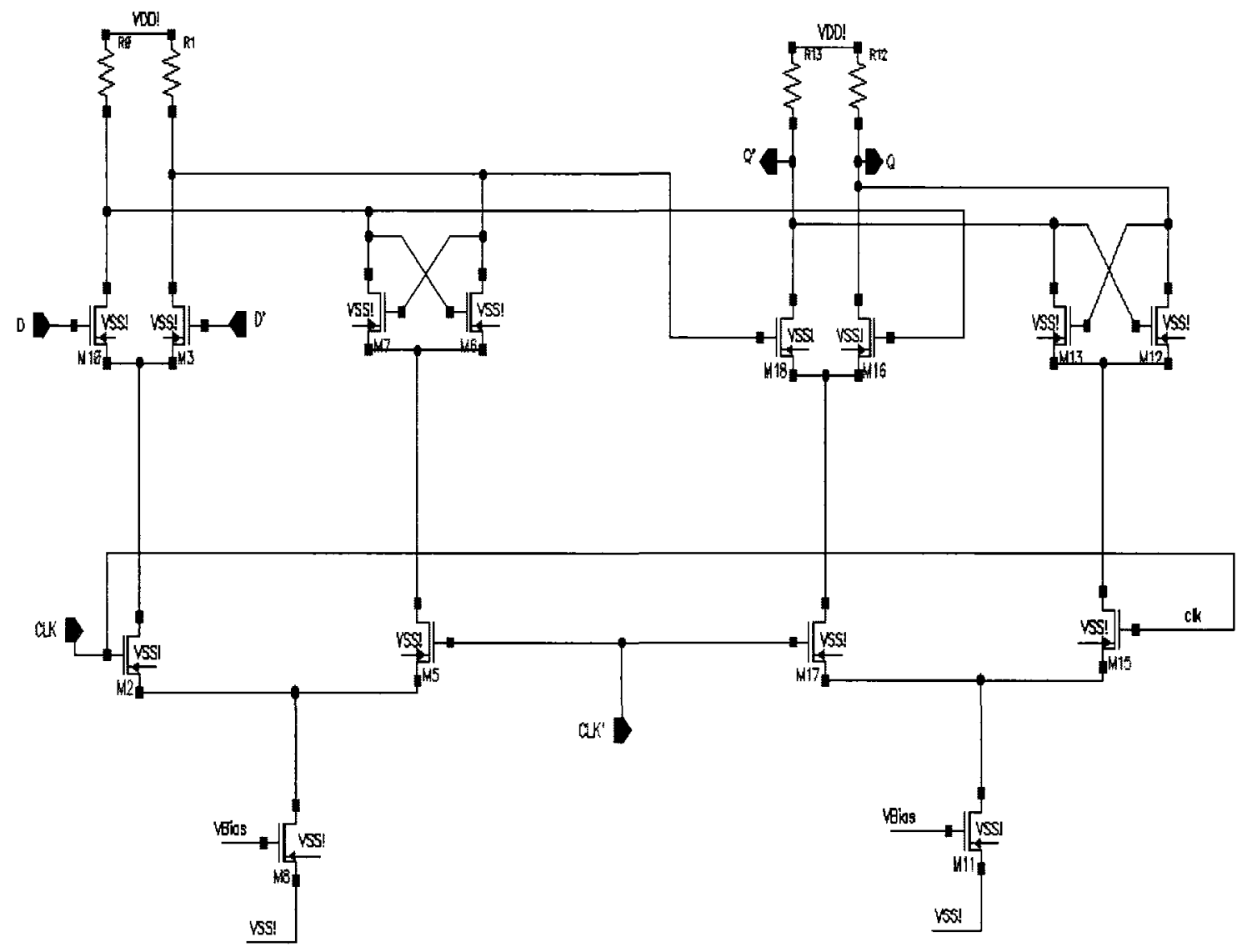

FIGURE B.1 D Flip-Flop 

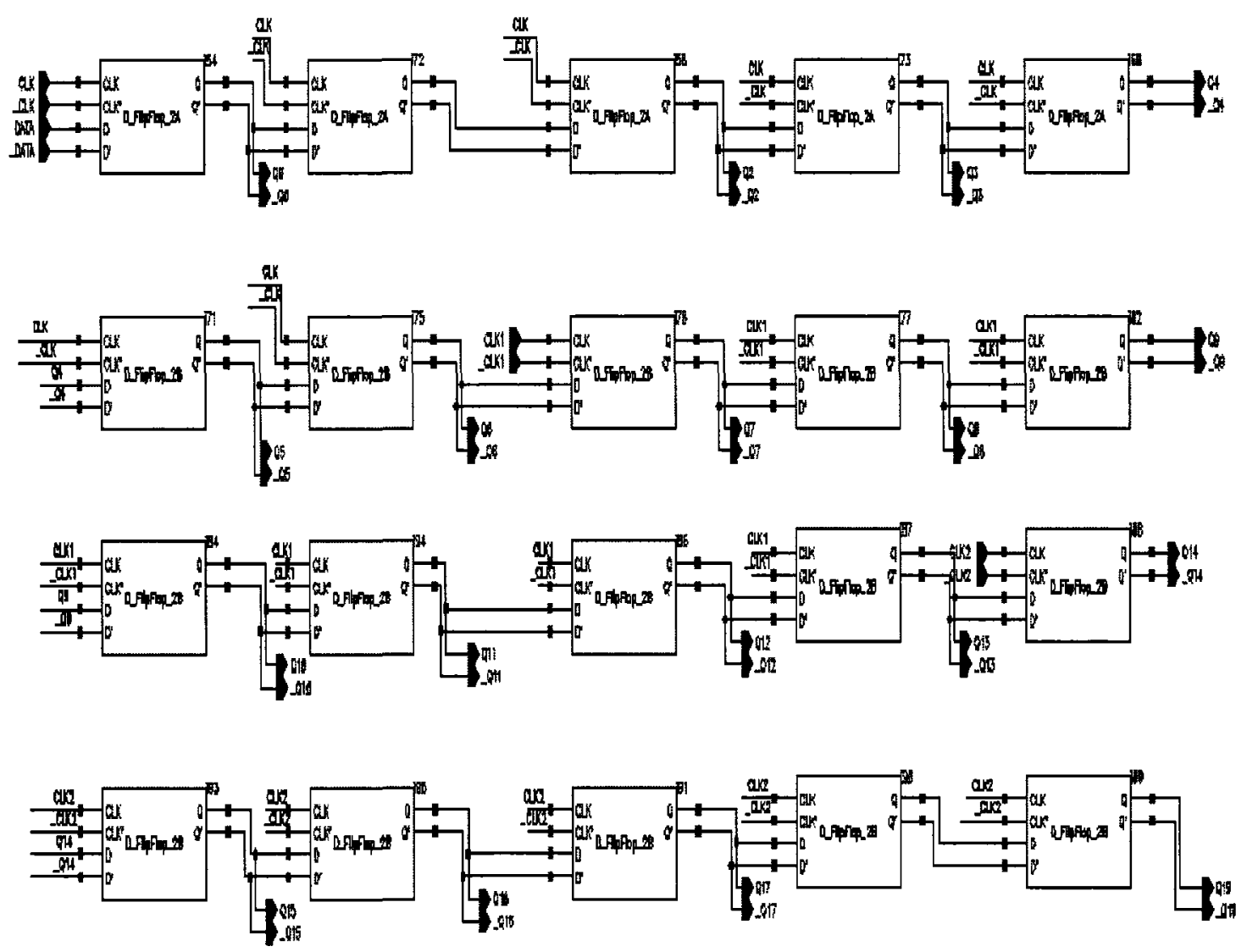

FIGURE B.2 Shift Register

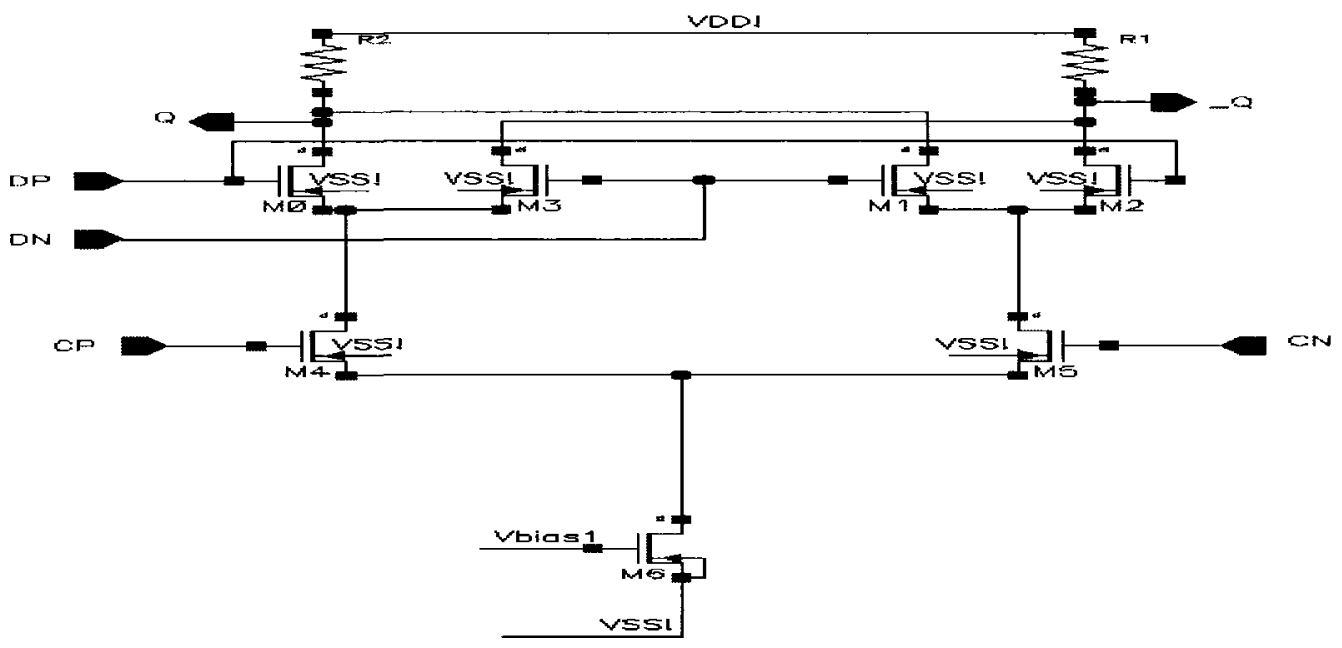

FIGURE B.3 XOR Gate

UWB Ultra-Low-Power Short Distance FIR Spectrally Shaped NRZ Transmission 


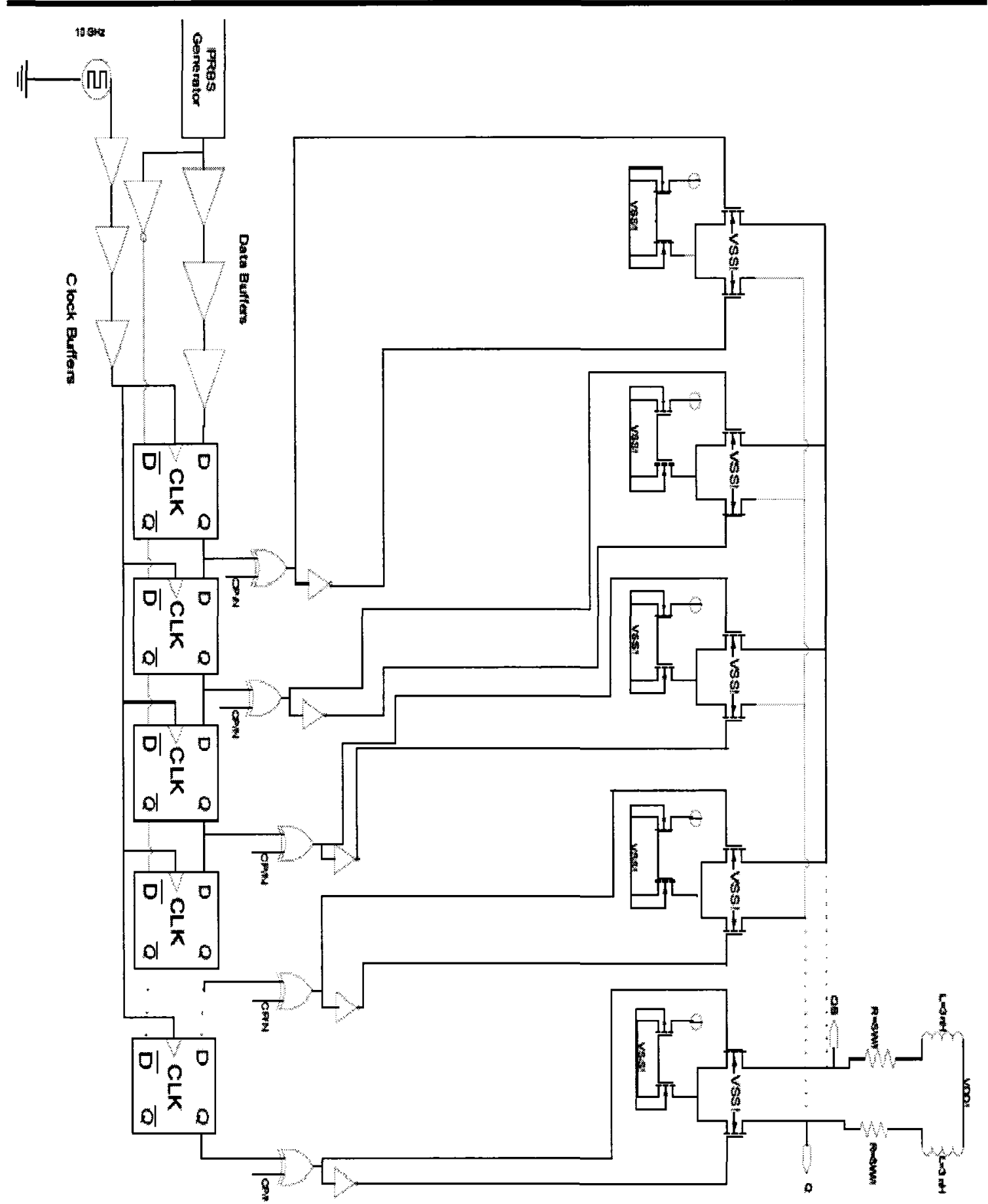

FIGURE B.4 Transmitter Block Diagram

The above block diagram is simplified . Originally, differential structure is used for gates and extracted inductor model is used. 


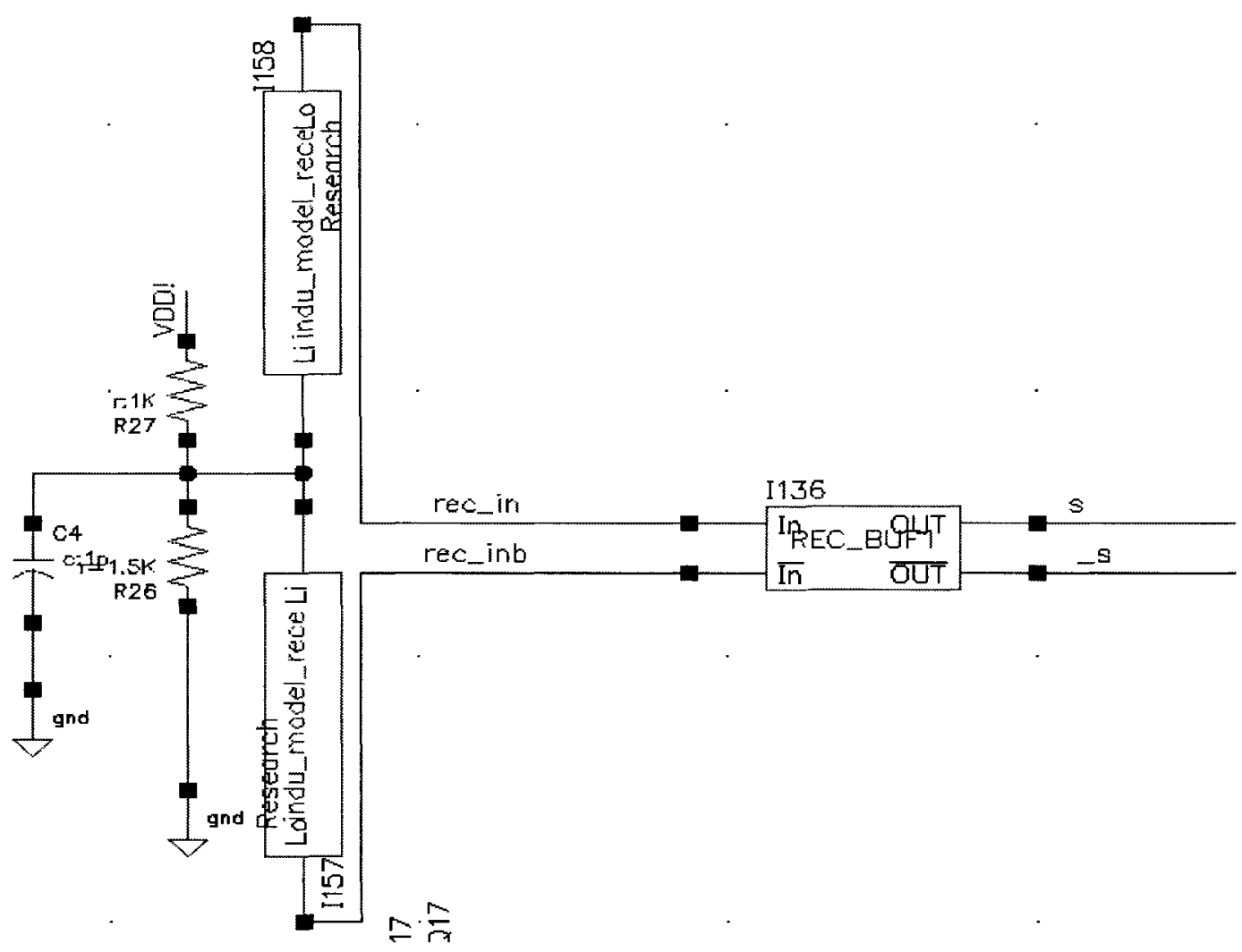

FIGURE B.5 Receiver Block Diagram

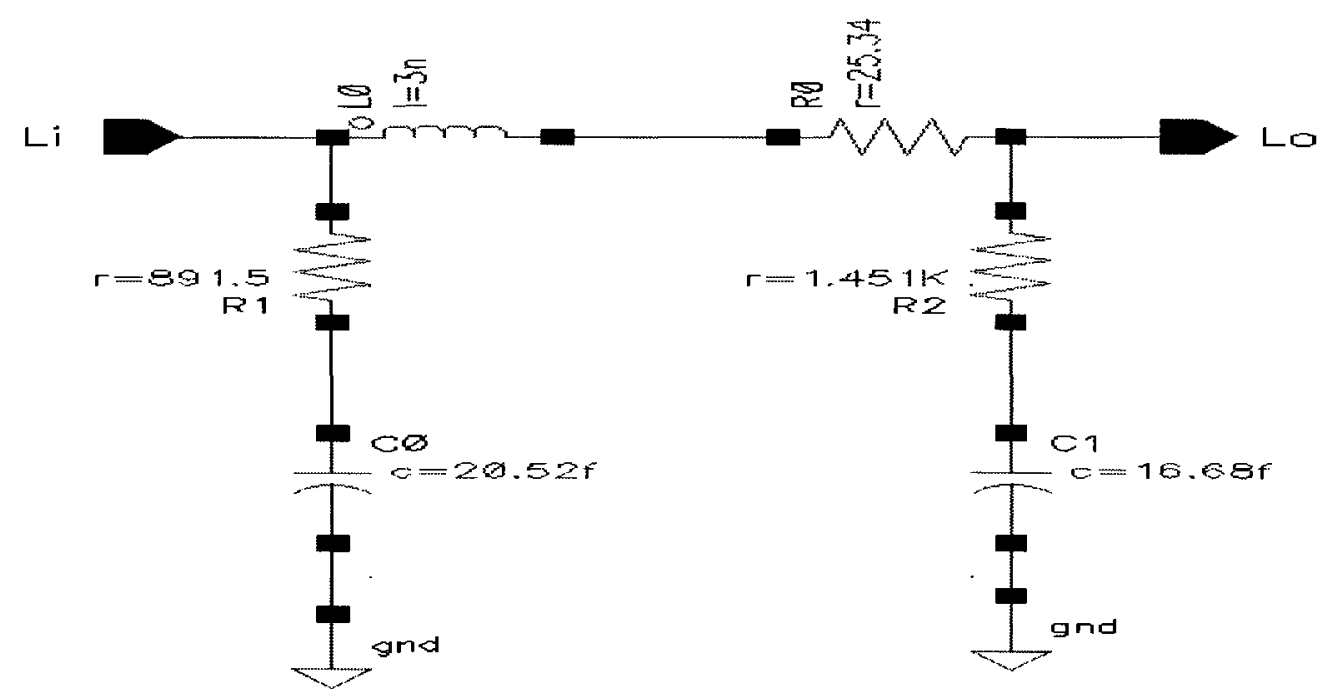

FIGURE B.6 Transmitter Inductor Model 
Li

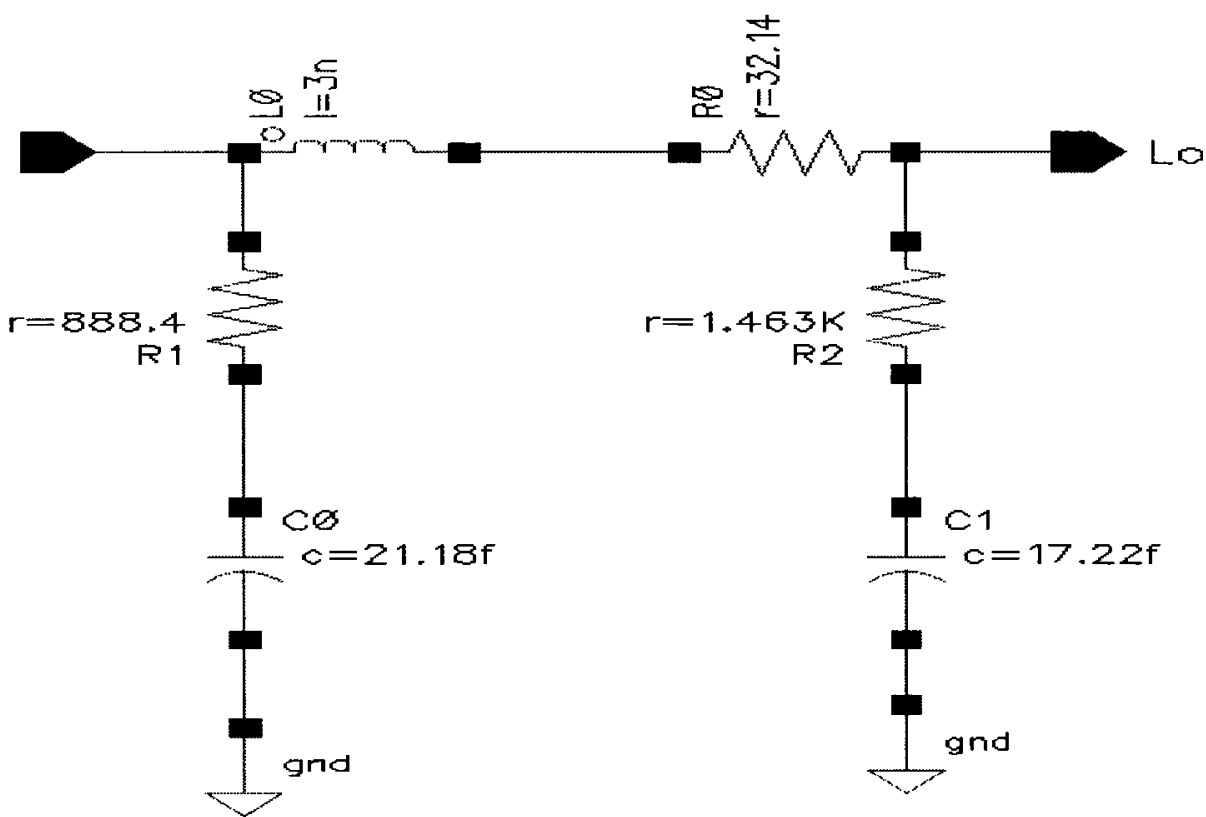

FIGURE B.7 Receiver Inductor Model

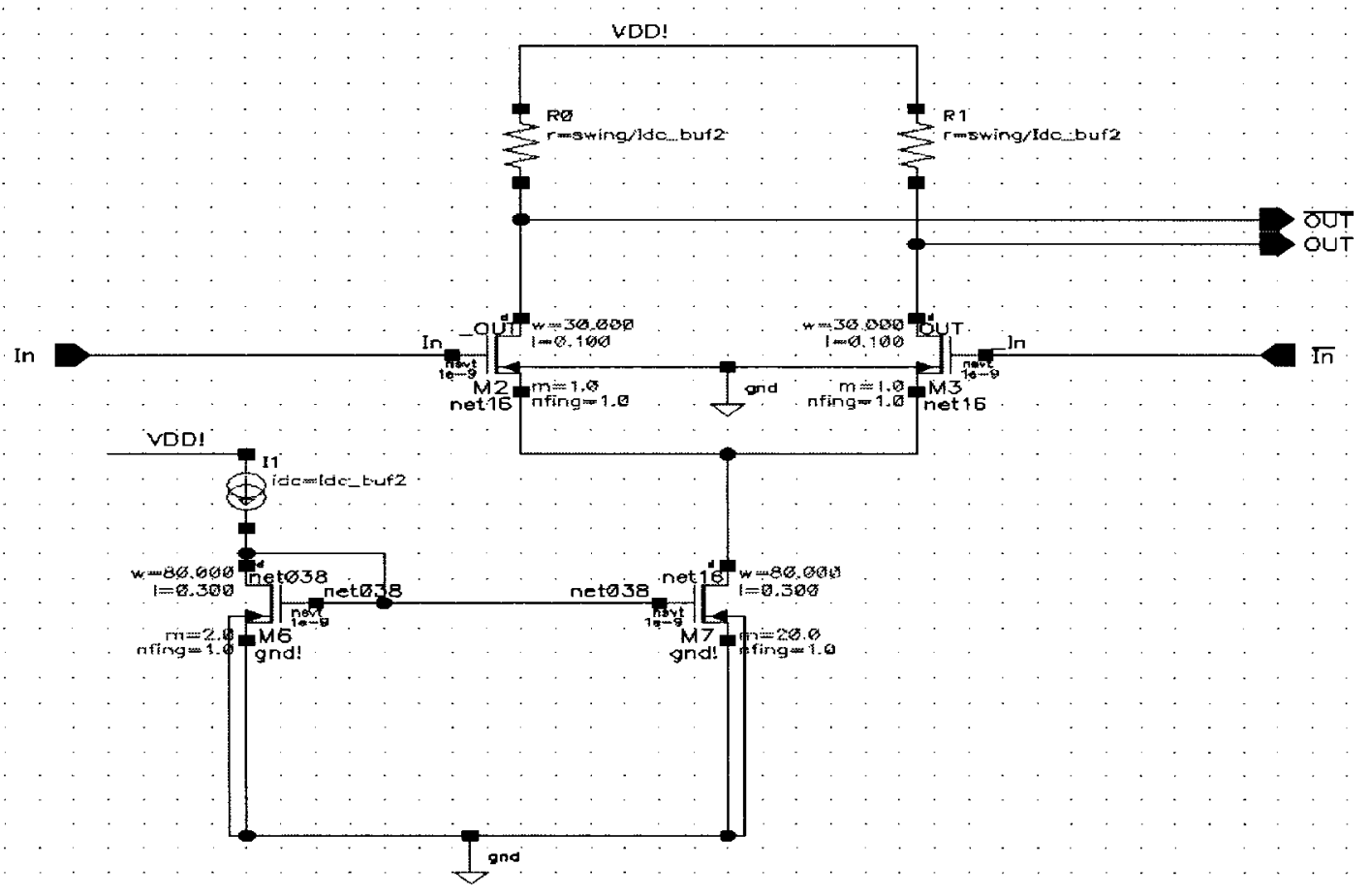

FIGURE B.8 Clock and Data Buffer $(I=1.15 \mathrm{~mA}, \mathrm{Sw}=0.4 \mathrm{~V})$

UWB Ultra-Low-Power Short Distance FIR Spectrally Shaped NRZ Transmission 


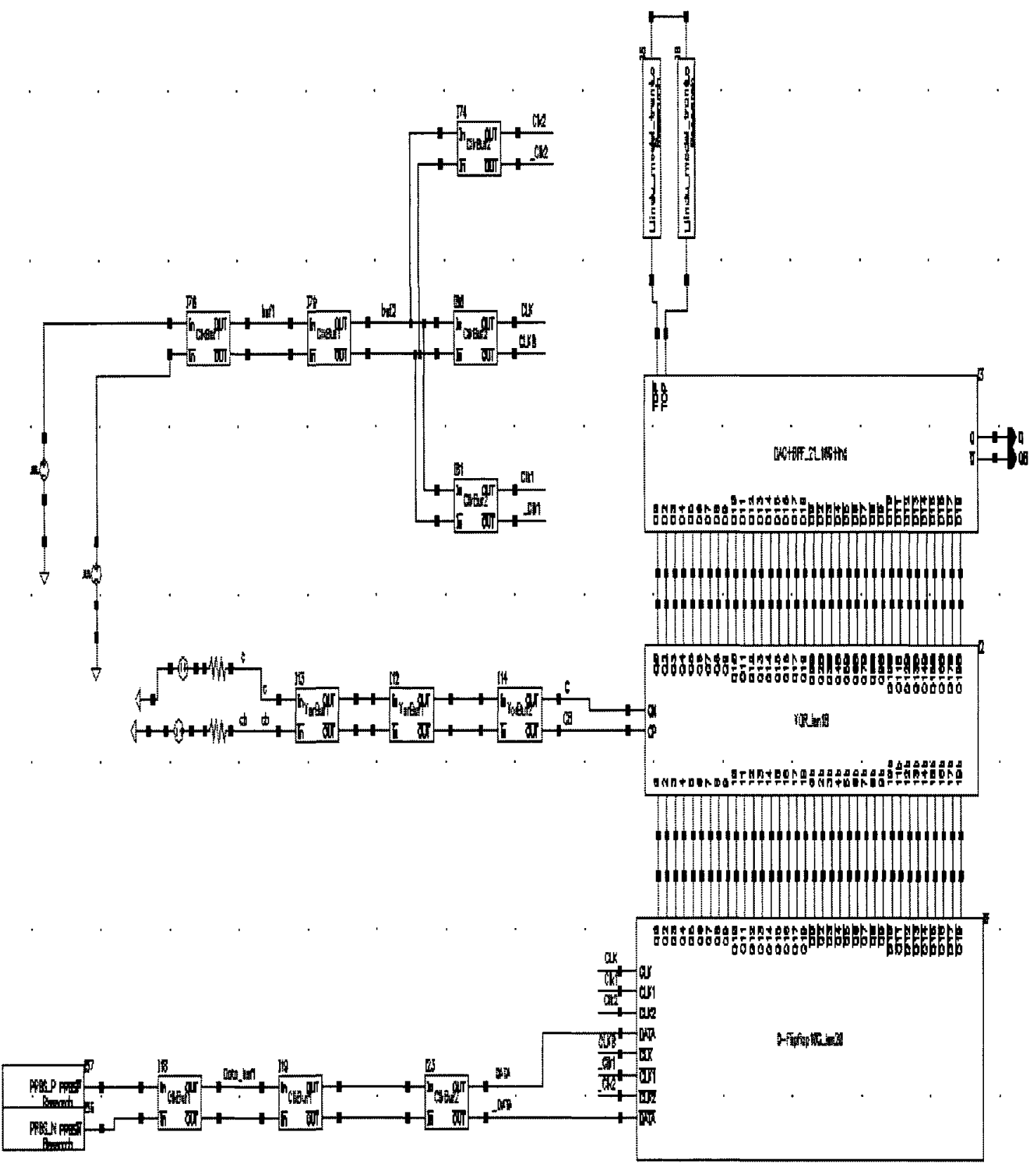

FIGURE B.9 Transmitter Test Bench 


\section{References}

1. Federal Communication Comission, Government Printing Office, Washington, "First Report and Order: Revision of part 15 of the commission's rules regarding ultra-wideband transmission systems," ET Docket 98-153 April 2002.

2. D. Culler, D. Estrin, M. Srivastava, "Guest editors' introduction: overview of sensor networks," IEEE Computer Magazine, issue 8, vol. 37, pp. 41- 49, August 2004.

3. T. C. Karalar, S. Yamashita, M. Sheets, J. Rabaey, "An integrated, low power localization system for sensor networks," IEEE Proceedings of The First Annual International Conference on Mobile and Ubiquitous Systems: Networking and Services, MOBIQUITOUS 2004, pp. 24-30, August 2004.

4. M. Shen, T. Koivisto, T. Peltonen, L. Zheng, E. Tjukanoff, H. Tenhunerf, "UWB radio module design for wireless sensor networks," IEEE Proceedings of 23rd NORCHIP Conference, pp. 184-187, November 2005.

5. Faranak Nekoogar, Ultra wideband communications fundamentals and applications, Prentice Hall Publication 2006.

6. Huseyin Arslan, Zhi Ning Chen, Maria- Gabriella Di Benedetto, Ultra wideband wireless communication, Wiley Interscience, John Wiley and Sons Inc. Publication 2006.

7. A. Batra et al., "Multi-band OFDM physical layer proposal for IEEE 802..15 task group 3a," IEEE 802.15-04/0493rl, September 2004.

8. S. Oh-Soon, S. Ghazzemzadeh, L. J. Greenstein, V. Tarokh, "Performance evaluation of MB-OFDM and DS-UWB systems for wireless personal area networks," IEEE Proceedings of International Conference on Ultra-Wideband, ICU 2005, pp. 214-219, September 2005.

9. L. Stocia, S. Tiuraniemi, I. Oppermann, H. Repo "An ultra wideband low complexity circuit transceiver architecture for sensor networks," IEEE Proceedings of International Conference on Circuits and Systems, ISCAS 2005, vol 1, pp. 364-367, May 2005. 
10. H. Kim, D. Park, and Y. Joo, "All-digital low-power CMOS pulse generator for UWB system," Electronics Letters, issue 24, vol. 40, pp. 1534-1535, November 2004.

11. S. Chang, S. Jung, S. Tjuatja, J. Gao, Y. Joo, "A CMOS 5th derivative impulse generator for an IR-UWB," IEEE Proceedings of 49th IEEE International Midwest Symposium on Circuits and Systems, MWSCAS 2006, vol. 2, pp. 376-380, August 2006.

12. P. Heydari, "Design considerations for low-power ultra wideband receivers," IEEE Proceedings of International Symposium on Quality of Electronic Design, ISQED 2005, pp. 668-673, March 2005.

13. I. D. O'Donnell, R.W. Brodersen, "An ultra-wideband transceiver architecture for low power, low rate, wireless systems," IEEE Transactions on Vehicular Technology, issue 5, vol. 54, pp. 1623-1631, September 2005.

14. W. Namgoong, "A channelized DSSS ultra-wideband receiver," Proceedings of IEEE Radio and Wireless Conference, RAWCON 2001, pp. 105-108, August 2001.

15. L. Hyung-Jin, H. Dong, L. Hyung-Soo, “A frequency domain approach for all digital CMOS ultra wideband receivers," Proceedings of IEEE Conference on Ultra Wideband Technologies, pp. 86-90, November 2003.

16. B. Razavi, T. Aytur, C. Lam, Y. Fei-Ran, L. Kuang-Yu, Y. Ran-Hong, K. Han-Chang, H. Cheng-Chung, L. Chao-Cheng, "A UWB CMOS transceiver," IEEE Journal of Solid-State Circuits, issue 12, vol 40, pp. 2555-2562, December 2005.

17. R. Blazquez, P. P. Newaskar, F. S. Lee, A. P. Chandrakasan, "A Baseband Processor for Pulsed UWB signal," Proceedings of the IEEE 2004 Custom Integrated Circuits Conference, pp. 587-590, October 2004.

18. L. Stoica, S. Tiuraniemi, A. Rabbachin, I. Oppermann, "An ultra wideband TAG circuit transceiver architecture," IEEE International Workshop on Ultra Wideband Systems, Joint with Conference on Ultrawideband Systems and Technologies, Joint UWBST \& IWUWBS 2004, pp. 258-262, May 2004.

19. T. Takahide, Y. Shingo, M. Muqsith, S. Yukitoshi, K. Tadahiro "A CMOS ultra wideband impulse radio transceiver for $1 \mathrm{Mb} / \mathrm{s}$ data communication and $+/-2.5 \mathrm{~cm}$ range finding," IEEE Journal of Solid-State Circuits, issue 4, vol 41, pp. 891-898, April 2006.

20. T. Yang, Y. Jiangnan, X. Yong-Ping, Y. Wooi Gan, L Fujiang, Yuanijinzheng, "A low power non-coherent CMOS UWB transceiver IC," IEEE Digest of Papers Radio Frequency integrated Circuits (RFIC) Symposium, pp. 347-350, June 2005.

21. L. Stoica, A. Rabbachin, I. Oppermann, "A low-complexity noncoherent IR-UWB transceiver architecture with TOA estimation," IEEE Transactions on Microwave Theory and Techniques, issue 4, part 2, vol.54, pp. 1637-1646, June 2006.

22.W. Zhongshan, H. Jianqiang, G. Guoxiang, "A new transceiver design for multi-band UWB system," Proceedings of IEEE International Conference on Acoustics, Speech, and Signal Processing, ICASSP 2005, vol. 3, pp. 633-636, March 2005. 
23. L.Yang, "Low-complexity diversity receiver for single/multi-band UWB," IEEE Workshop on Signal Processing Advances in Wireless Communications, pp. 10531057, June 2005.

24. M. S. W. Chen, R.W. Brodersen, "A subsampling UWB radio architecture by analytic signaling," Proceedings of IEEE International Conference on Acoustics, Speech, and Signal Processing, ICASSP 2004, vol.4, pp. 533-536, May 2004.

25. John G. Proakis, Digital Communications, Prentice Hall Publication 1995.

26.M. Li, T. Kwasnieski, S.Wang, and Y. Tao, "A 10Gb/s transmitter with multi-tap FIR pre-emphasis in 0.18 CMOS technology," IEEE Proceedings of Asia South Pacific Design Automation Conference, ASP-DAC 2005, pp. 679-682, January 2005.

27. S. Rylov, S. Reynolds, D. Storaska, B. Floyd, M. Kapur, T. Zwick, S. Gowda, M. Sorna, "10+Gb/s 90nm CMOS serial Link Demo in CBGA Package," IEEE Journal Of Solid State Circuits, vol. 40, issue 9, pp. 1987 - 1991, September 2005.

28. R. Farjad-Rad, C. K. K. Yang, M. A. Horowitz, T. H. Lee, “A 0.4- $\mu \mathrm{m}$ CMOS 10-Gb/s 4-PAM pre-emphasis serial link transmitter," IEEE Journal of Solid State Circuits, vol. 34, no. 5, pp. 580-585, May 1999.

29. John G. Proakis, Digital Signal Processing, Prentice Hall Publication 1996.

30. R. J. Drost, R. D. Hopkins, R. Ho, I. E. Sutherland, "Proximity communication," IEEE Journal of Solid State Circuits, issue 9, vol. 39, pp. 1529-1535, September 2004.

31. J. Ryckaert, G. Van der Plas, V. De Heyn, C. Desset, B. Van Poucke, J. Craninckx, “A 0.65-to-1.4 nJ/Burst 3-to-10 GHz UWB all-digital TX in $90 \mathrm{~nm}$ CMOS for IEEE 802.15.4a," IEEE Journal of Solid-State Circuits, vol. 42, issue 12, pp. 2860-2869, December 2007.

32. T. Kikkawa, P. K. Saha, N. Sasaki, K. Kimoto, "Gaussian monocycle pulse transmitter using $0.18 \mu \mathrm{m}$ CMOS technology with on-chip integrated antennas for inter-chip UWB communication,' IEEE Journal of Solid-State Circuits, vol.43, issue 5, pp. 13031312, May 2008.

33. A. Medi, W. Namgoong, "A high data-rate energy-efficient interference-tolerant fully integrated CMOS frequency channelized UWB transceiver for impulse radio," IEEE Journal of Solid-State Circuits, vol. 43, issue 4, pp. 974-980, April 2008.

34. L. Smaini, C. Tinella, D. Helal, C. Stoecklin, L. Chabert, C. Devaucelle, R. Cattenoz, N. Rinaldi, D. Belot, "Single-chip CMOS pulse generator for UWB systems," IEEE Journal of Solid-State Circuits, vol. 41, issue 7, pp. 1551-1561, July 2006.

35. C. Liang, S. -T. Liu, S. -I. Liu, "A calibrated pulse generator for impulse-radio UWB applications," IEEE Journal of Solid-State Circuits, vol. 41, issue 11, pp. 2401-2407, November 2006.

36. S. Lo, I. Sever, S. Ma, P. Jang, A. Zou, C. Arnott, K. Ghatak, A. Schwartz, L. Huynh, V. Phan, T. Nguyen, "A dual-antenna phased-array UWB transceiver in $0.18 \mu \mathrm{m}$ 
CMOS," IEEE Journal of Solid-State Circuits, vol.41, issue 12, pp. 2776-2786, December 2006.

37. T. Norimatsu, R. Fujiwara, M. Kokubo, M. Miyazaki, A. Maeki, Y. Ogata, S. Kobayashi, N. Koshizuka, K. Sakamura, "A UWB-IR transmitter with digitally controlled pulse generator," IEEE Journal of Solid-State Circuits, vol. 42, issue 6, pp. 13001309, June 2007.

38. M. Demirikan, R. R. Spencer, "A 1.8 Gpulses/s UWB transmitter in 90nm CMOS," IEEE Proceedings of International Solid-State Circuits Conference, ISSCC 2008, pp. 116-117, Febuary 2008.

39. Z. N. Chen, " UWB Antennas : Design and Application," IEEE Proceedings of International Conference on Information, Communications and Signal Processing, ICICS 2007, pp.1-5, December 2007. 